Revue des patrimoines

$10 \mid 2009$

Le patrimoine scientifique

\title{
Les chemins de fer atmosphériques. Première partie
}

\section{Paul Smith}

\section{(2) OpenEdition \\ 12 Journals}

Édition électronique

URL : http://journals.openedition.org/insitu/4192

DOI : $10.4000 /$ insitu.4192

ISSN : 1630-7305

Éditeur

Ministère de la Culture

Référence électronique

Paul Smith, "Les chemins de fer atmosphériques. Première partie », In Situ [En ligne], 10 | 2009, mis en ligne le 19 mai 2009, consulté le 14 novembre 2019. URL : http://journals.openedition.org/insitu/4192

Ce document a été généré automatiquement le 14 novembre 2019.

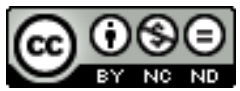

In Situ Revues des patrimoines est mis à disposition selon les termes de la licence Creative Commons Attribution - Pas d'Utilisation Commerciale - Pas de Modification 4.0 International. 


\title{
Les chemins de fer atmosphériques. Première partie
}

\author{
Paul Smith
}

\section{Avant-propos}

1 En France, les chemins de fer modernes tels que nous les concevons, avec des locomotives tirant des trains sur des rails, sont nés dans la région de Saint-Étienne pour le transport du charbon vers une voie d'eau'. En 1832, sur la ligne de $18 \mathrm{~km}$ ouverte en 1827 entre Saint-Étienne et Andrézieux, sur la Loire, des locomotives commençaient à remplacer la traction animale et les trains à prendre des voyageurs payants. Dans le nord de l'Angleterre, sensiblement à la même époque, une autre ligne d'origine houillère, reliant Darlington au port fluvial de Stockton-on-Tees et conçue à l'origine, en 1825, pour des trains tractés par des chevaux ou des locomotives, commençait à être exclusivement exploitée avec ces dernières, sauf pour deux plans inclinés sur lesquels les wagons étaient tirés par des cordes, grâce à des machines à vapeur fixes. La réussite de cette ligne incita à en ouvrir une autre, en 1830, entre Liverpool et Manchester, également conçue pour le transport de marchandises, essentiellement des matières premières textiles destinées aux filatures et tissages de Manchester et de sa région. Mais cette "grande » ligne de $56 \mathrm{~km}$ de longueur, comportant elle aussi deux rampes où la traction s'effectuait par câble, rencontra un succès tout à fait inattendu dans le transport de passagers. Considérée comme le véritable précurseur des chemins de fer modernes, reliant deux grandes villes avec des trains de voyageurs et de marchandises exploités par une compagnie unique et respectant des horaires réguliers, cette ligne célèbre inspira une pléthore de nouveaux projets, sans véritable plan d'ensemble mais donnant à l'Angleterre une incontestable longueur d'avance dans la construction des chemins de fer. En 1840, l'Angleterre comptait $3600 \mathrm{~km}$ de chemins de fer achevés ou en cours d'exécution ${ }^{2}$, quand la France n'en comptait que 400, comprenant la ligne de Saint-Étienne à Lyon, le chemin d'Andrézieux à Roanne, celui de Montpellier à Sète et les premières « lignes-réclame » quittant Paris en direction de Saint-Germain-en-Laye et de Versailles, et destinées exclusivement au transport de voyageurs. 
2 Mettant fin à de longs débats idéologiques, économiques et techniques, la loi du 11 juin 1842, partagea, en principe, la responsabilité de la réalisation des chemins de fer français entre l'État, chargé des dépenses de construction de l'infrastructure, et des compagnies concessionnaires ayant à leur charge celles de la superstructure : la voie, les gares, le matériel roulant et l'exploitation. La même loi consacra aussi le dessin radial des grandes lignes françaises, s'étendant de Paris vers les frontières continentales et maritimes. Ce dessin, dit étoile de Legrand, du nom du sous-secrétaire d'État aux Travaux publics, allait marquer durablement l'espace du territoire tout entier ainsi que celui de la capitale, confortée dans sa prééminence démographique, économique, industrielle, politique et intellectuelle ${ }^{3}$. À la suite de ce tournant de 1842, la construction des chemins de fer prit un véritable essor en France : en 1850, il y avait $3000 \mathrm{~km}$ de voies. À partir de 1845 se développa également une industrie autochtone de construction de locomotives, notamment à Paris mais aussi à Mulhouse, au Creusot, à La Ciotat et à Lyon. À la fin de l'année 1849, sur 1013 locomotives roulant en France, 850 y avaient été construites ${ }^{4}$. À l'Exposition universelle de Londres de 1851, des locomotives présentées par les constructeurs français pouvaient soutenir, sans désavantage, la comparaison avec celles d'Angleterre ${ }^{5}$.

Toutefois, au cours de ces premières décennies de la révolution ferroviaire, les locomotives à vapeur, malgré de rapides et notables perfectionnements, étaient loin de représenter aux yeux de tout le monde le dernier mot en matière de traction ferroviaire. Leur construction était bien plus coûteuse que celle des machines à vapeur fixes. Peu fiables, elles étaient coûteuses aussi d'entretien et d'exploitation, gaspillant une grande partie de leur énergie dans la traction de leur propre poids et celui de leur tender. Elles ne consommaient que du coke, plus cher que le charbon brûlé sous les chaudières des machines fixes. Charriant feu et eau bouillante, semant cendres, escarbilles et fumée, elles étaient souvent perçues comme menaçantes, voire explosives et incendiaires. Dès 1830, d'ailleurs, elles avaient fait la preuve de leur dangerosité. Si on ne connaît pas d'éventuels accidents mortels arrivés à des mécaniciens ou à d'autres ouvriers anonymes de ces premiers chemins de fer, le premier survenu à un passager, William Huskisson, membre des Communes pour Liverpool et président du Board of Trade, renversé par le Rocket de George et de Robert Stephenson lors de l'inauguration du Liverpool-Manchester le 15 septembre 1830, est fort bien documenté. Le drame avait manifesté d'emblée l'une des caractéristiques de l'accident ferroviaire qui frappait les contemporains, son absence d'égard pour le rang de ses victimes. De nombreux accidents sont connus par la suite, le plus retentissant et de loin le plus meurtrier étant celui survenu à Meudon le 8 mai 1842, catastrophe qui coûta la vie à une soixantaine de personnes dans l'incendie des voitures qui suivit la rupture d'essieu d'une locomotive et le déraillement de son train, au retour des Grandes Eaux de Versailles ${ }^{6}$. Parmi les victimes, le célèbre explorateur Dumont d'Urville et sa famille, ainsi que le mécanicien anglais Thomas George, ancien directeur des ateliers de construction des Stephenson à Newcastle-upon-Tyne, responsable depuis 1838 du matériel de la Compagnie du chemin de fer de Paris à Versailles (Rive gauche) et conducteur de l'une des deux locomotives? De tels accidents mettaient en cause la machine elle-même, dans laquelle on commençait à observer et à étudier la fatigue des métaux. Mais ils mettaient aussi en cause la responsabilité des ouvriers chargés des mouvements quotidiens des locomotives, les mécaniciens surtout, fiers sans doute et indépendants à leur poste de conduite, mais assez mal payés et, le cas échéant, ivres ou fous, voire les deux ${ }^{8}$. Pour 
certains observateurs, le système à locomotives était tout simplement un système « homicide " .

4 Pour des raisons relevant à la fois de cette brûlante question de sécurité et de celle de l'économie d'ensemble du système à locomotives, les chemins de fer ne pouvaient être construits qu'au prix de lourds travaux d'infrastructure. La faible adhérence des roues aux rails interdisait l'usage des premières locomotives sur de fortes pentes qui, lorsqu'elles ne pouvaient pas être évitées, imposaient le recours à des systèmes de traction par corde ou par câble, enroulé sur des tambours par des machines à vapeur fixes. Pour conserver l'avantage fabuleux de la vitesse tout en limitant les risques de déraillement, les courbures des voies devaient avoir de très grands rayons, de mille mètres au minimum selon l'administration des Ponts et Chaussées. D'où les ouvrages d'art - tranchées, tunnels, remblayages, viaducs - permettant au tracé de s'écarter le moins possible de l'idéal: une ligne rigoureusement droite et horizontale. D'où également les débats contradictoires, particulièrement vifs autour des avant-projets pour la ligne de Paris à Rouen et au Havre ${ }^{10}$, entre le tracé direct « par les plateaux » et le tracé par la vallée, plus sinueux mais desservant mieux les centres et les intérêts urbains existants. D'où aussi, enfin, la difficulté pour les compagnies, notamment à Paris, mais également à Londres, d'établir leurs embarcadères où elles l'auraient souhaité, au cœur de la ville.

5 Ainsi, au cours des années qui sont celles en France de la Monarchie de Juillet, des technologies alternatives de chemins de fer florissaient, proposant des solutions au seul problème des courbures ou, plus radicalement, des forces propulsives inusitées, celles de l'air comprimé ou de l'air raréfié, c'est-à-dire un vide partiel. Cet article propose un examen de ces alternatives, dans lesquelles certains ingénieurs, et non des moindres, pensaient avoir trouvé enfin le parangon des chemins de fer. Il cherche tout d'abord, et tout simplement, à rappeler l'histoire de cet épisode atmosphérique, peu connu en dehors des spécialistes des chemins de fer. À travers un récit chronologique des quatre lignes atmosphériques commerciales mises en place en Irlande, en Angleterre et en France, il vise à restituer quelque chose de l'enthousiasme qui entoura le nouveau système à ses débuts. Il posera, en conclusion, deux questions plus larges que cet épisode peut éclairer. La première s'intéresse aux raisons pour lesquelles certaines innovations techniques « marchent » et d'autres sont des échecs. La deuxième concerne la circulation des connaissances techniques dans la première moitié du XIX siècle, l'éclosion de ce que Louis Bergeron a appelé «une culture commune de l'ingénieur européen ", et la place de la France dans cette culture ${ }^{11}$. En post-scriptum, un bilan des vestiges physiques des chemins de fer atmosphériques sera dressé. Mais l'article s'ouvre avec un bref rappel des idées mises en avant, du XVII ${ }^{e}$ siècle au début du XIXe, pour l'usage de l'air comme moyen de transmission des forces physiques.

\section{L'énergie de l'air}




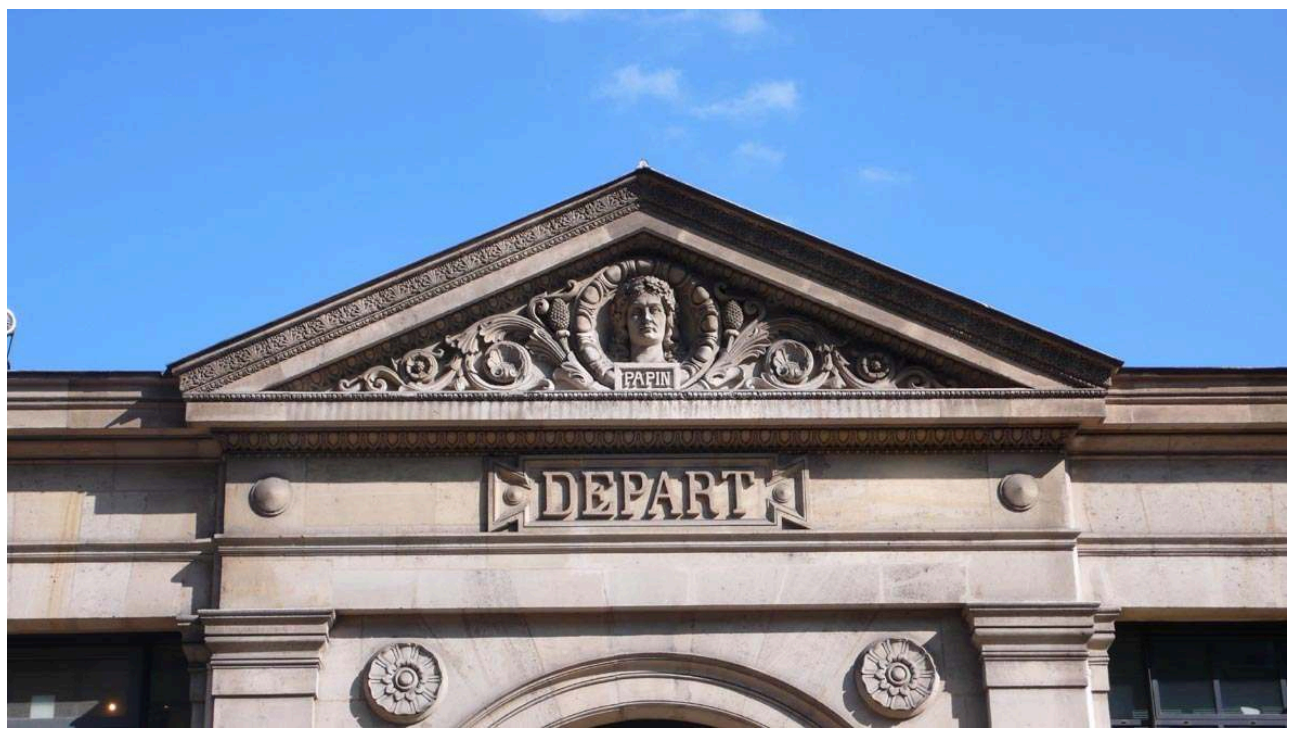

Portrait sculpté de Denis Papin ornant le bâtiment départ de la Gare du Nord à Paris (1864) ; un second fronton présente le buste de James Watt. Photo Paul Smith, 2008.

(c) Paul Smith.

Lorsqu'on ne la fait pas remonter aux vents qui gonflent les voiles des navires ou encore au premier siècle après Jésus-Christ, époque à laquelle Héron d'Alexandrie aurait appliqué la dilatation de l'air chauffé pour ouvrir les portes d'un temple, l'histoire canonique de la transmission de l'énergie par l'air commence avec l'invention de la pompe à air par le physicien allemand Otto von Guericke, et sa célèbre expérience des hémisphères de Magdebourg, en 1650. Ensuite, le savant d'origine irlandaise Robert Boyle améliora la pompe à air en s'intéressant plus généralement aux rapports entre la pression et le volume d'un gaz. Mais c'est à «l'immortel» Denis Papin ${ }^{12}$ (fig. $\left.\mathbf{n}^{\circ} \mathbf{1}\right)$, associé de Robert Boyle et membre comme lui de la Société royale de Londres, que l'on attribue, à la fin du XVII siècle, l'idée d'exploiter le ressort de l'air - sa capacité d'être comprimé ou raréfié par des pompes - pour transmettre la force à distance, dans des tubes.

7 Préoccupé comme Boyle par les questions portant sur la pression de l'air et sur son absence, le "vuide», Papin s'est notamment intéressé aux moyens de "presser le vent » dans un tuyau pour maintenir une flamme dans un vaisseau plongé sous l'eau, idée annonciatrice de la cloche de plongeur mise au point par l'astronome Edmond Halley vers 1716. Papin s'est également appliqué à faire entrer de l'air - soixante fois plus qu'il n'y en avait à l'ordinaire - dans un vaisseau de verre, cet air comprimé (c'est l'expression qu'il emploie) faisant un bruit pareil à celui d'une grenade lorsqu'il fit rompre le verre. Et c'est en imaginant la création du vide par la condensation de la vapeur dans un cylindre sous un piston soumis au poids de l'atmosphère qu'il put avancer sa théorie d'une «machine pour avoir à bon marché de grandes forces mouvantes ». Ces forces, remarque-t-il, pour expliquer leur bon marché, seraient préférables à celles des galériens qui non seulement chargent la galère et occupent beaucoup de place, mais qu'il faut toujours nourrir, qu'ils travaillent en mer ou qu'ils se reposent dans les ports ${ }^{13}$.

Mais, comme pour la machine à vapeur, il fallut attendre des progrès dans les arts mécaniques pour voir une application pratique des idées de Papin en matière de 
transmission d'énergie par l'air. C'est à la célèbre manufacture de Matthew Boulton et James Watt à Soho, près de Birmingham, l'un des principaux foyers de ce qu'il est convenu d'appeler la révolution industrielle ${ }^{14}$, que l'air aurait vu ses premiers usages pour la transmission de la force. Employé dans cette manufacture à partir de 1777, l'inventeur écossais William Murdoch, connu surtout comme pionnier de l'éclairage au gaz $^{15}$, parvint à faire marcher une machine de l'atelier des modèles à l'aide d'air comprimé par une soufflerie de la forge. Il construisit aussi un monte-charge pneumatique pour lever les moulages de fonte du sol de l'atelier placé au bord du canal et inventa également un système de transport de paquets dans des tubes par l'effet de l'air comprimé ${ }^{16}$. L'air comprimé actionnait en outre les sonneries à Sycomore House, la maison qu'il se fit construire en 1817.

9 En France, la fin des années 1830 connut quelques essais d'application de l'air comprimé, notamment pour le fonçage des puits de mines. Vers 1839, à Chalonnes (Maine-et-Loire), l'ingénieur civil Triger utilisa l'air comprimé dans ses travaux pour atteindre un terrain houiller reposant sous vingt mètres d'alluvions de la Loire. Son système, un perfectionnement en quelque sorte de la cloche de plongeur mais permettant de pénétrer dans des terrains solides, comportait un tube étanche en tôle de fer de douze millimètres d'épaisseur et d'un mètre de diamètre intérieur, fabriqué (à Paris) par sections de cinq à six mètres de longueur. Non sans difficultés, ce tube fut enfoncé dans les sables au moyen d'un mouton d'un poids de deux tonnes. L'appareil à air comprimé proprement dit comprenait une machine à vapeur et deux pompes comprimant l'air dans le tube pour en refouler l'eau. Un trou d'homme doté d'un presse-étoupe servait d' "écluse à air ", permettant l'introduction des ouvriers dans le tube et l'extraction des déblais. Avant d'y envoyer ses hommes, Triger expérimentait lui-même la possibilité de travailler sous une pression de trois à quatre atmosphères, partageant avec l'Académie des Sciences ses observations sur les différents phénomènes observés ${ }^{17}$ : une douleur plus ou moins vive se manifestant dans les oreilles, mais qui cessait avec le rétablissement de l'équilibre entre l'air comprimé à l'intérieur du tube et l'air renfermé dans l'oreille interne; l'accélération, dans l'air comprimé, de la combustion des chandelles; le froid sensible produit par la distension de l'air comprimé ; le fait que, dans l'air comprimé, il n'est plus possible à personne de siffler, qu'un mineur sourd - le nommé Floc - aurait entendu plus distinctement que ses camarades, qu'en montant dans les échelles tous les ouvriers se trouvaient moins essoufflés qu'à l'air libre... Au-delà de ces constats de nature physique et physiologique, Triger entrevoyait déjà de nombreuses applications pratiques pour son appareillage, non seulement dans les mines mais aussi dans les travaux de génie maritime, pour le creusement des ports ou dans la construction des ponts.

Et, en effet, dans les années suivantes, l'emploi de l'air comprimé pour les travaux d'établissement des piles des ponts se répandit rapidement, notamment en Angleterre au pont de Rochester sur le Medway, réalisé par l'ingénieur William Cubitt en $1851^{18}$. Le célèbre ingénieur Isambard Kingdom Brunel, déjà familiarisé avec les cloches de plongeur lors de ses travaux en 1827 au tunnel sous la Tamise conçu par son père, utilisa également ce qu'il appelait la «méthode pneumatique " pour la fondation des piles de ses ponts ferroviaires sur le Wye à Chepstow, entre 1849 et 1852, et sur le Tamar - le Royal Albert Bridge - à Saltash, de 1854 à $1859^{19}$. 
Figure 2
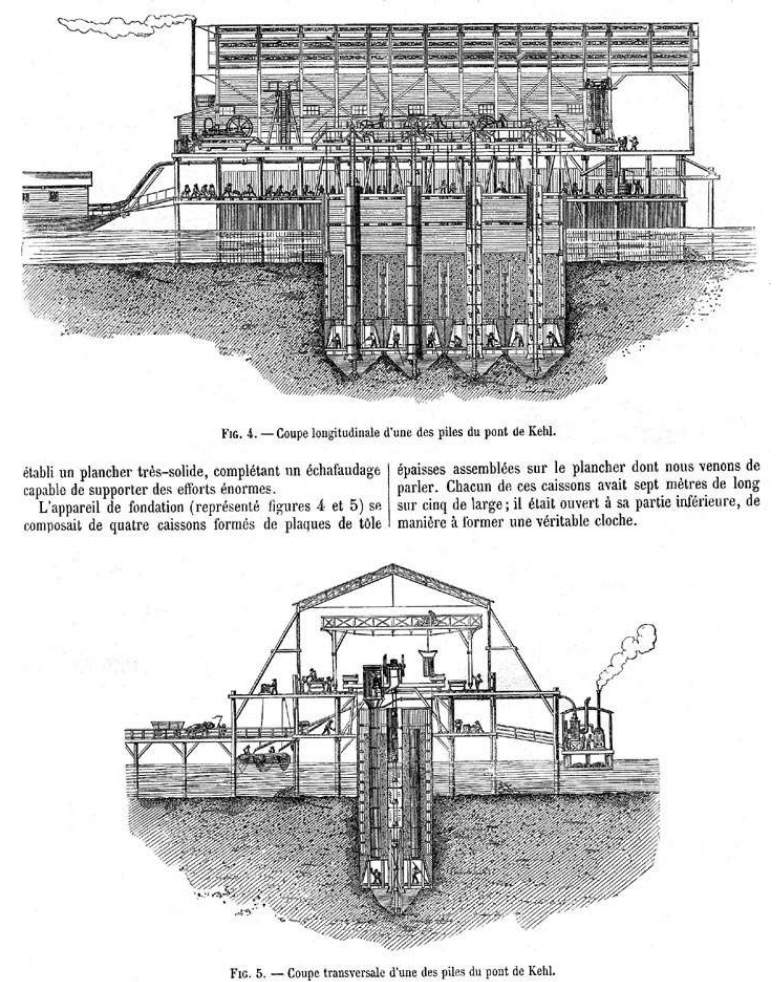

Travaux de fondation des piles et culées du pont de Kehl, LE MAgASIN PITTORESQUE, 1862, p. 191. Repro Paul Smith, 2008.

(C) Paul Smith.

Réalisés par l'entreprise Cail de février à décembre 1859, les travaux de fondation des quatre piles du pont ferroviaire de Kehl entre la France et le Grand-duché de Bade, à vingt mètres sous le niveau des basses eaux du Rhin, mobilisèrent aussi l'air comprimé, envoyé non pas dans un tube ou un "grand cylindre » mais dans des caissons accolés, mis au point par les ingénieurs Émile Vuigner ${ }^{20}$ et Édouard Fleur, dit Fleur Saint-Denis ${ }^{21}$ (fig. $\mathrm{n}^{\circ} 2$ ).

En 1845, à Chalonnes, de nouveau, dans une innovation encore plus intéressante, l'ingénieur Triger avait utilisé l'air comprimé pour la transmission de la force motrice. Mû par une machine à vapeur installée à la surface, un compresseur envoyait l'air par des canalisations à une machine située à l'intérieur des travaux souterrains, à $230 \mathrm{~m}$ de distance. Plus docile que la vapeur, sec et ne menaçant pas dès lors les boisages des galeries par un apport d'humidité, ne faisant que peu de bruit et, surtout, ne semant sur son passage ni feu ni fumée, l'air comprimé s'imposa comme un agent avantageux pour la mécanisation des travaux miniers. Rapidement connues, ses promesses incitèrent d'autres houillères à s'équiper en compresseurs : dès 1849 dans les houillères de Govan, près de Glasgow; en 1863, aux mines de Sars-Longchamp et Bouvy en Belgique $^{22}$; en 1866, aux mines royales de Saarbruck; en 1868, aux mines de Marihaye dans le bassin de Liège et aux mines d'Anzin en France... Fonte, cuivre, fer étiré, tôle rivée, plomb et caoutchouc furent expérimentés pour les canalisations tandis que quelques entreprises - Derosne et Cail, Farcot et fils, Rouquayrol-Denayrouze, Sautter et Lemonnier - commençaient à se spécialiser dans la construction de compresseurs. Chargé en 1866 par la Compagnie des Mines d'Anzin d'étudier les moyens mécaniques 
utilisés dans les mines en Angleterre, M. A. Pernolet rendit hommage aux travaux pionniers de son compatriote Triger, constatant «que l'air comprimé était l'agent mécanique le plus souple et le plus avantageux pour transmettre et distribuer à l'intérieur des travaux la force motrice qu'il est le plus souvent impossible de produire dans les chantiers aux points mêmes où elle doit être utilisée ${ }^{23}$ ».

\section{Les chemins de fer atmosphériques, premiers projets}

\section{George Medhurst}

13 Cloches de plongée, fondations des piles des ponts, travaux miniers... Vu l'époque, il n'est guère surprenant qu'on ait pensé très tôt à mettre cette force de l'air au service des transports ${ }^{24}$. La première proposition dans ce sens aurait été formulée par l'Anglais George Medhurst, né en 1759 à Shoreham, dans le Kent, et qui était fabricant d'instruments de pesage à Londres, dans le quartier de Soho. Ses projets sont en fait antérieurs à l'avènement des chemins de fer et se présentent plutôt comme une alternative aux transports par canal, interrompus deux mois sur douze par le gel, les inondations ou la sécheresse. Après deux premiers brevets pris en 1799 et 1800 pour un " engin éolique » à air comprimé25, Medhurst publia en 1810 une brochure d'une douzaine de pages intitulée A new method of conveying letters and goods with great certainty and rapidity by air ${ }^{26}$. Cette méthode nouvelle consistait en un tube ou tunnel construit en brique, en pierre, en bois ou en fer, ayant une section de douze pieds carrés et à l'intérieur duquel un véhicule à quatre roues, roulant sur un chemin de fonte ou de pierre, serait propulsé par l'air comprimé, produit à la bouche du tunnel par une machine à vapeur ayant la force de dix-huit chevaux. Dans une deuxième brochure, de $1812^{27}$, l'inventeur, conscient de l'inconvénient de confiner des passagers dans le noir à l'intérieur d'un tube, sans aucune vue sur les campagnes traversées, propose un tube plus petit, de section rectangulaire, dans lequel il y aurait une boîte mobile, ou piston, attaché à un véhicule à l'extérieur au moyen d'un "particular contrivance». Ce « dispositif particulier» maintiendrait la communication entre le piston et la voiture sans laisser échapper l'air d'impulsion. Au titre des avantages de ce nouveau mode de transport, Medhurst évoque sa rapidité (50 milles à l'heure, ou $80 \mathrm{~km} / \mathrm{h}$ ), son bas coût (un farthing, ou quart de penny, par mille) et la possibilité de convoyer, outre des passagers ordinaires, des pièces d'artillerie, des troupes, du bétail, du poisson frais et du courrier, et ceci sans crainte ni des neiges, ni des inondations, ni des voleurs. Dans une brochure publiée en $1827^{28}$, Medhurst donne quelques gravures illustratives de ses différents projets (fig. $n^{\circ} 3, n^{\circ} 4, n^{\circ} 5, n^{\circ} 6, n^{\circ} 7$ ). 
Figure 3

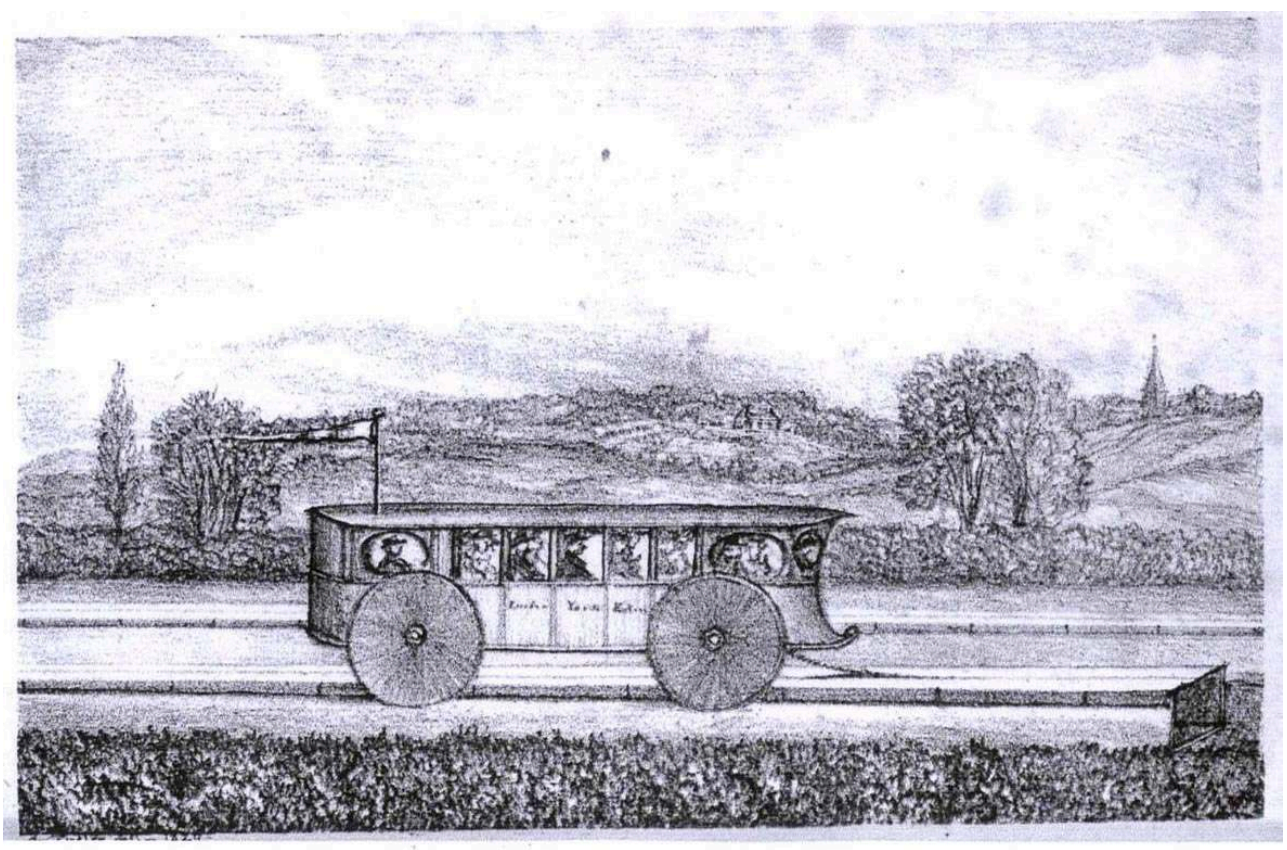

MEDHURST, George. A New SYSTEM OF INLAND ConveyANCE, 1827. Dessin de G. Butler servant de frontispice à la brochure. II s'agit d'un véhicule routier tracté par un piston à l'intérieur d'un tube de section carrée. Sur la carrosserie, on déchiffre London - York - Edinburgh.

(C) Institution of Civil Engineers.

Figure 4

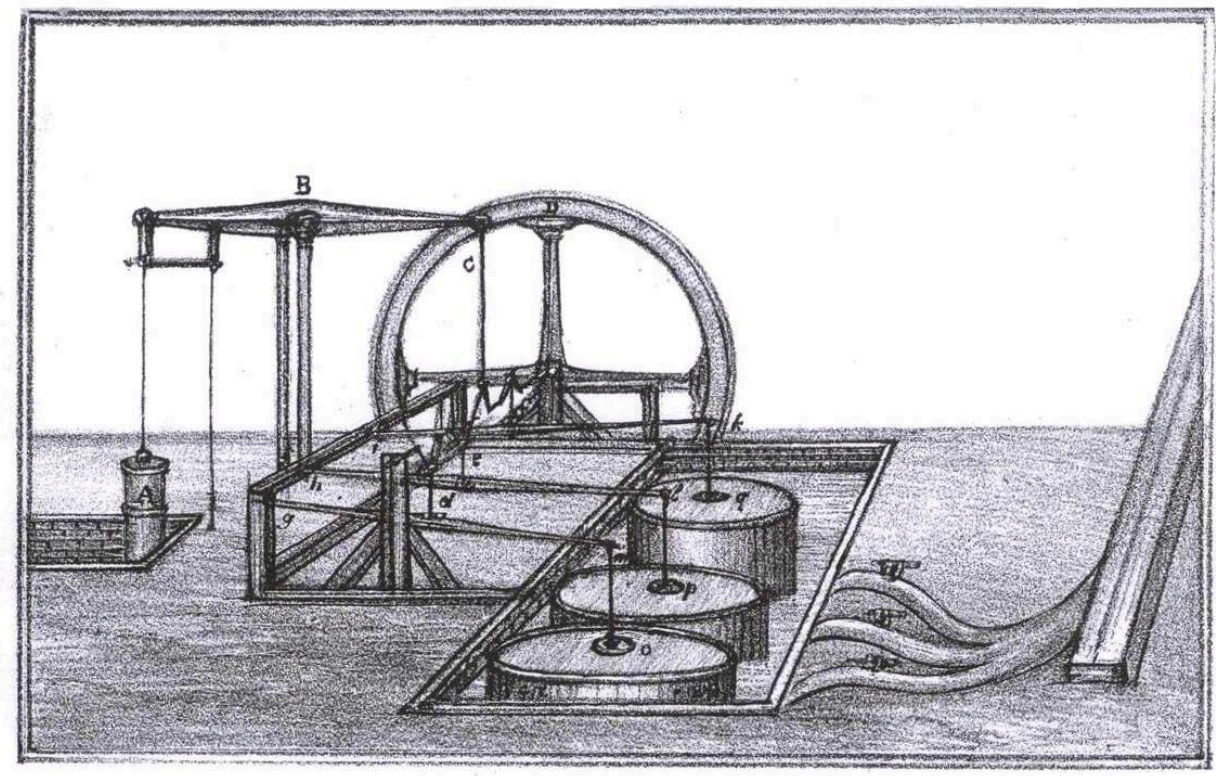

MEDHURST, George. A NEW SYSTEm OF INLAND CONVEYANCE, 1827. Disposition de la machine à vapeur destinée à insuffler l'air dans le tube ou pour l'en extraire. Les cuves des pompes sont construites en bois et cerclées de fer.

(C) Institution of Civil Engineers. 
Annonçant rien de moins que l'aube d'une époque nouvelle dans l'histoire de l'humanité, ceux-ci allaient contribuer aux richesses, à la splendeur, à la liberté, au bonheur, à la science et à la civilisation du monde entier.

Figure 5

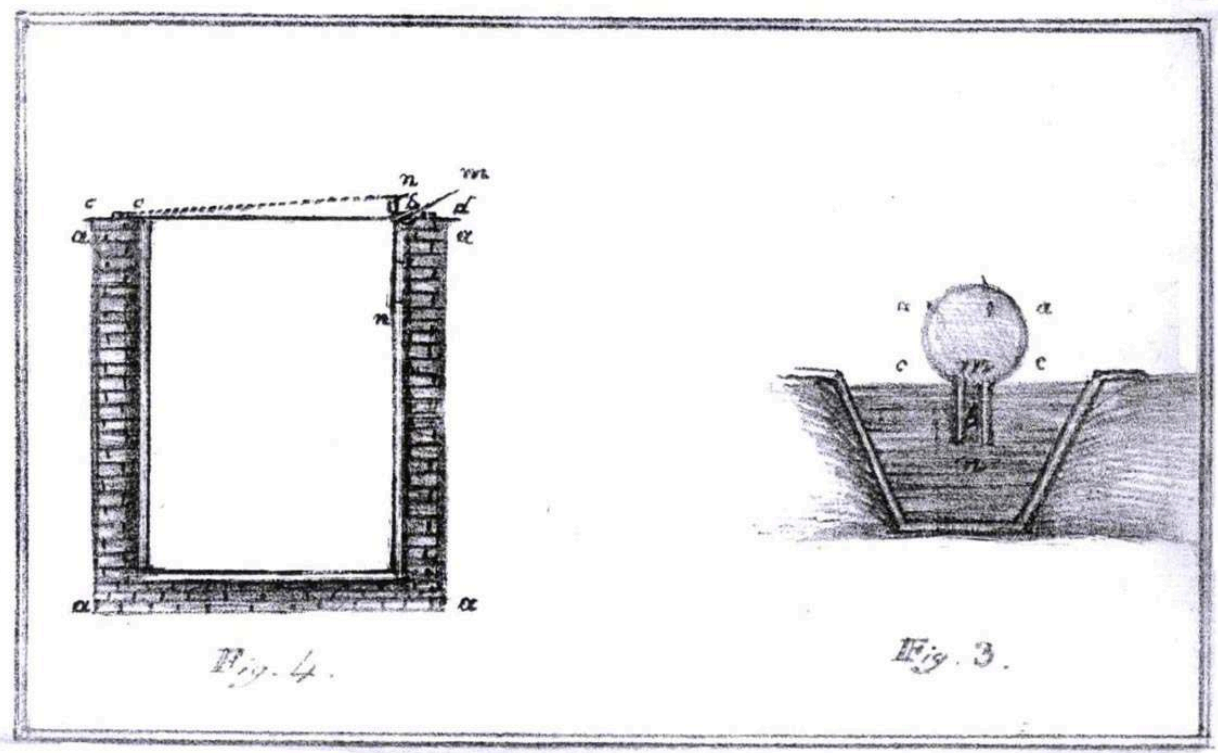

MEDHURST, George. A New SYSTEM OF INLAND CONVEyANCE, 1827. (Figure 3) : Tube en fer de 24 pouces de diamètre $(61 \mathrm{~cm})$, partiellement immergé dans un canal rempli d'eau. (Figure 4) : Section d'un tube carré couvert d'une plaque en fer qui se soulève pour laisser passer un bar en fer attaché à un véhicule.

(c) Institution of Civil Engineers.

Il imagine déjà un voyageur qui prendrait son petit déjeuner à Londres pour dîner ensuite à Edimbourg. Il réfléchit aussi, et de manière plus sérieuse, à la liaison entre le piston dans le tube et le véhicule situé à l'extérieur, liaison qui pourrait être effectuée à travers une espèce de soupape continue réalisée avec une matière molle, telle que liège, bois, cuir ou chanvre. Une autre de ses idées ici est d'effectuer le lien étanche entre piston et véhicule à travers l'eau, le tube étant partiellement immergé dans un étroit canal. Il reconnaît toutefois qu'il est obligatoire d'établir ce canal sur un plan rigoureusement horizontal, ce qui nécessite des écluses pour changer de niveau, écluses que le train pourrait franchir par sa propre vélocité. Medhurst envisage également un trafic dans les deux sens, soit par l'impulsion de l'air comprimé, soit par la raréfaction de l'air devant le piston, celui-ci étant aspiré en quelque sorte par le vide partiel, ou, plus précisément, propulsé par la pression atmosphérique de l'air admis derrière le piston. 
Figure 6

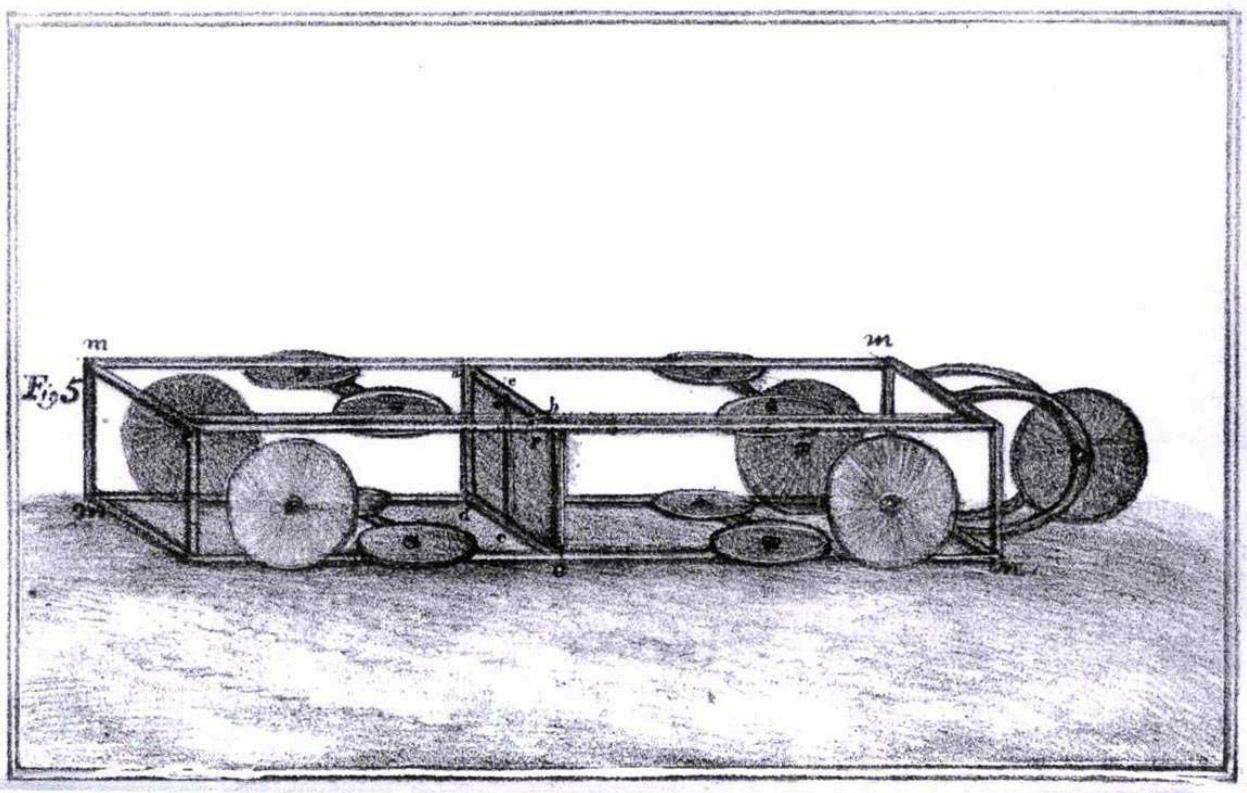

MEDHURST, George. A New SYSTEM OF INLAND CONVEYANCE, 1827. (Figure 5) : Le piston (abcd) dans son châssis $(\mathrm{mmmm})$, doté de roues horizontales et verticales.

(c) Institution of Civil Engineers.

Figure 7

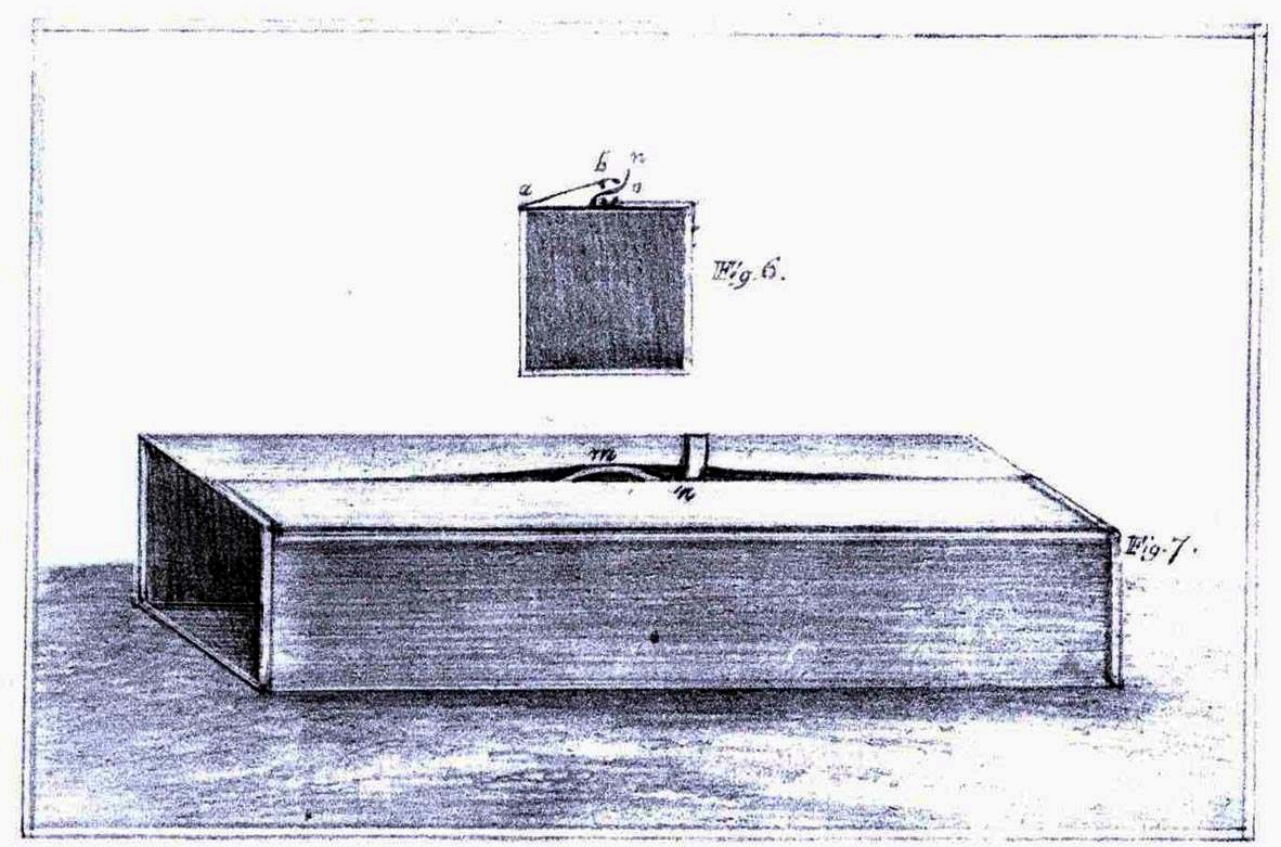

MEDHURST, George. A NEW SYSTEM OF INLAND CONVEYANCE, 1827. (Figure 6) : Section du tube avec un couvercle en fer forgé riveté à son bord et soulevé par la roue du châssis à l'intérieur. (Figure 7) : Longueur du tube avec son couvercle soulevé à $m$ pour laisser passer le bras $n$.

(c) Institution of Civil Engineers.

Malgré ses appels à des investisseurs, et malgré la fertilité de son imagination - on lui connaît d'autres brevets, par exemple pour des écluses de canal, et il prétend avoir fait 
marcher en 1820 un véhicule routier mû par une machine à vapeur -, Medhurst ne semble avoir réalisé aucun de ses projets de convoiement rapide par l'air. Sa brochure de 1827, l'année de sa mort, tient du testament. Il y revient sur une vie passée en adoration devant "l'invisible déité aérienne, une étrangère céleste aux vertus virginales ». Sa propre invention, son "bijou mécanique ", n'est dévoilée que pour l'admiration et la jouissance des âges futurs...

\section{John Vallance}

Dans une brochure publiée en $1824^{29}$ - à la veille, donc, de l'ouverture du chemin de fer entre Stockton et Darlington -, un autre inventeur anglais, John Vallance, qui n'avait apparemment pas connaissance des idées de Medhurst ${ }^{30}$, proposa de nouveau un système de propulsion atmosphérique, citant le précédent technique des trois cents milles de tubes en fonte installés depuis quelques années à Londres pour l'éclairage au gaz. Par extrapolation, il imagine une longueur comparable de cylindres en fonte, mais formant un tube de six pieds de diamètre $(1,83 \mathrm{~m})$ au lieu de six pouces $(15,24 \mathrm{~cm})$, dans lequel une voiture sur rails pourrait voyager, à la manière d'un piston, grâce au vide créé par pompage de l'air à l'une des extrémités. Il annonce d'emblée des vitesses de plusieurs centaines de milles à l'heure à des coûts d' $1 / 150^{\mathrm{e}}$ de farthing par tonne et par mille. Parmi les avantages de son système, il note en particulier ceux qui concernent la défense du royaume : la facilité avec laquelle des troupes pourraient être envoyées vers un littoral menacé. À la différence de Medhurst, cependant, Vallance put mettre ses idées en pratique. En 1826, dans son jardin à Brighton, il construisit un tube de cent cinquante pieds de longueur $(45 \mathrm{~m})$ et de huit pieds de diamètre $(2,4 \mathrm{~m})$, réalisé en planches de bois de sapin et recouvert de canevas pour le rendre étanche. À l'intérieur, sur des rails, une voiture de vingt-deux pieds de long $(6,7 \mathrm{~m})$, aspirée par le vide créé par des pompes à air actionnées par deux machines à vapeur, pouvait transporter six ou huit passagers. Le modèle fonctionnait, le trajet s'effectuant en une cinquantaine de secondes. 


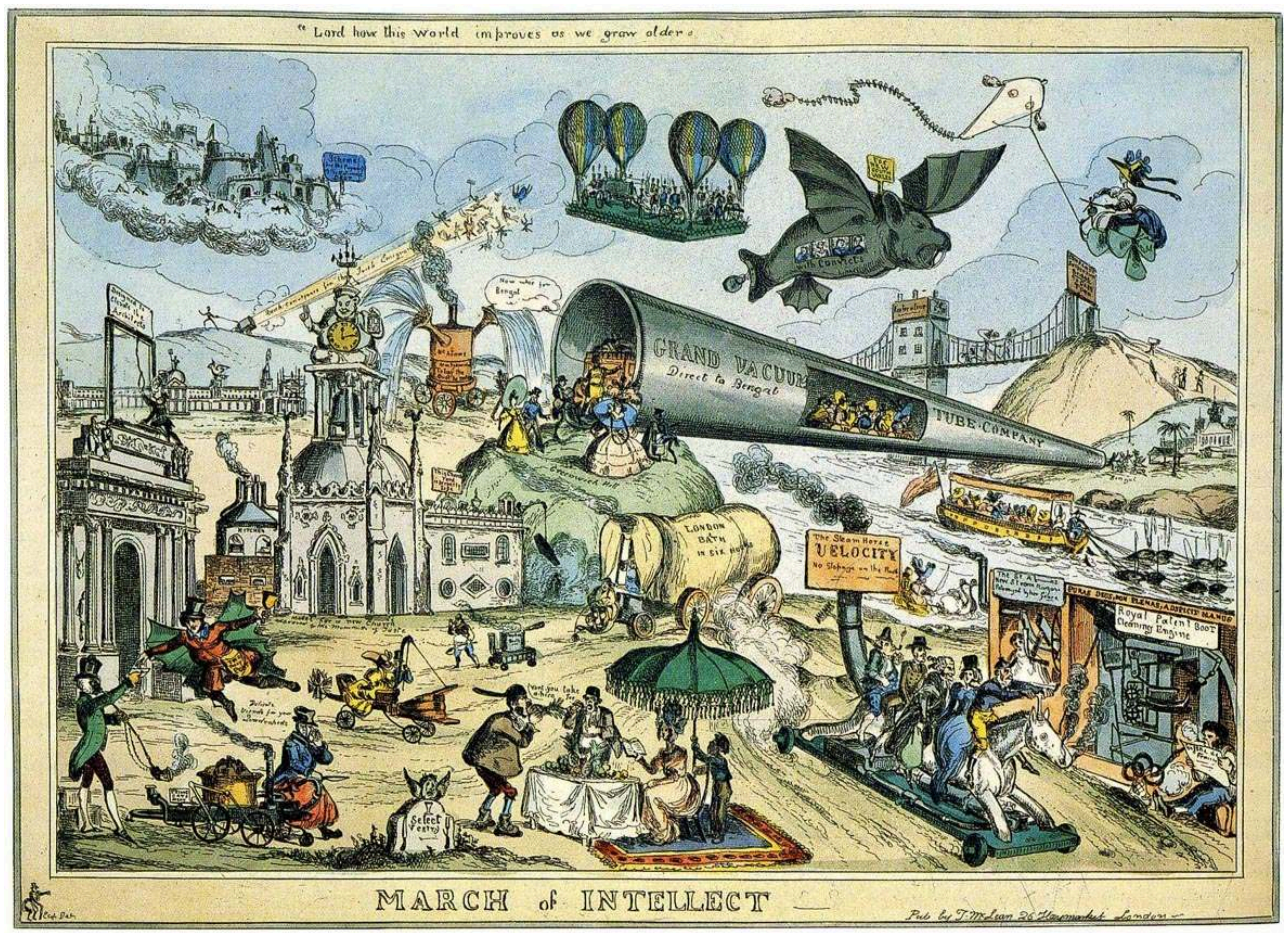

Paul Pry, March of Intellect, 1829. À l'époque des projets de John Vallance, ce dessin tourne en dérision les progrès de l'intellect et les innombrables projets mus par la vapeur. Le Grand Vacuum Tube Company promet d'emmener des voyageurs directement de Londres (Greenwich Hill) au Bengale. Reproduit par FREEMAN, Michael J. Railways and the Victorian IMAgINATION, 1999, p. 60. Repro Paul Smith, 2008.

(c) Paul Smith.

Il fut essayé par Sir William Couling, ingénieur au service de l'ambassade russe, par le comte de Flahaut ${ }^{31}$ ainsi que par une succession de "noble Dukes, Lords, Ladies, Gentlemen and learned Physicians ${ }^{32}$ ». Soutenu par la ville, qui, rappelons-le, était alors une station balnéaire à la mode, Vallance lança aussitôt un prospectus pour «The London, Brighton and Shoreham Pneumatic Conveyance Company ${ }^{33}$, estimant à $£ 500000$ (environ 12,5 millions de francs de l'époque) le coût d'installation d'un chemin pneumatique entre Brighton et la métropole. Avec 200000 passagers par an, un bénéfice annuel de $€ 150000$ pouvait être escompté. Mais aucun acte du Parlement ne vint autoriser ce chemin inédit, dont le projet, assez facilement tourné en dérision, sombra dans l'oubli (fig. $\mathbf{n}^{\circ} \mathbf{8}$ ).

\section{Henry Pinkus}

Peu de temps après, et de manière débarrassée de rêves de grandeur instantanée, un troisième précurseur, Henry Pinkus, ingénieur et spéculateur originaire de Pennsylvanie mais résidant à Londres, s'intéressait également à ce qu'il appelait «le chemin de fer pneumatique ». Son premier brevet, pris en $1834^{34}$, reprend l'une des idées de Medhurst en imaginant la création d'un vide partiel dans un tube en fonte de quarante-deux pouces de diamètre (un mètre environ), fabriqué par longueurs de quinze à vingt pieds (de 4,5 à $6 \mathrm{~m}$ ) et intégrant les rails, de trois pouces de largeur $(7,6 \mathrm{~cm})$, sur ses bords (fig. $\mathbf{n}^{\circ}$ 9). 


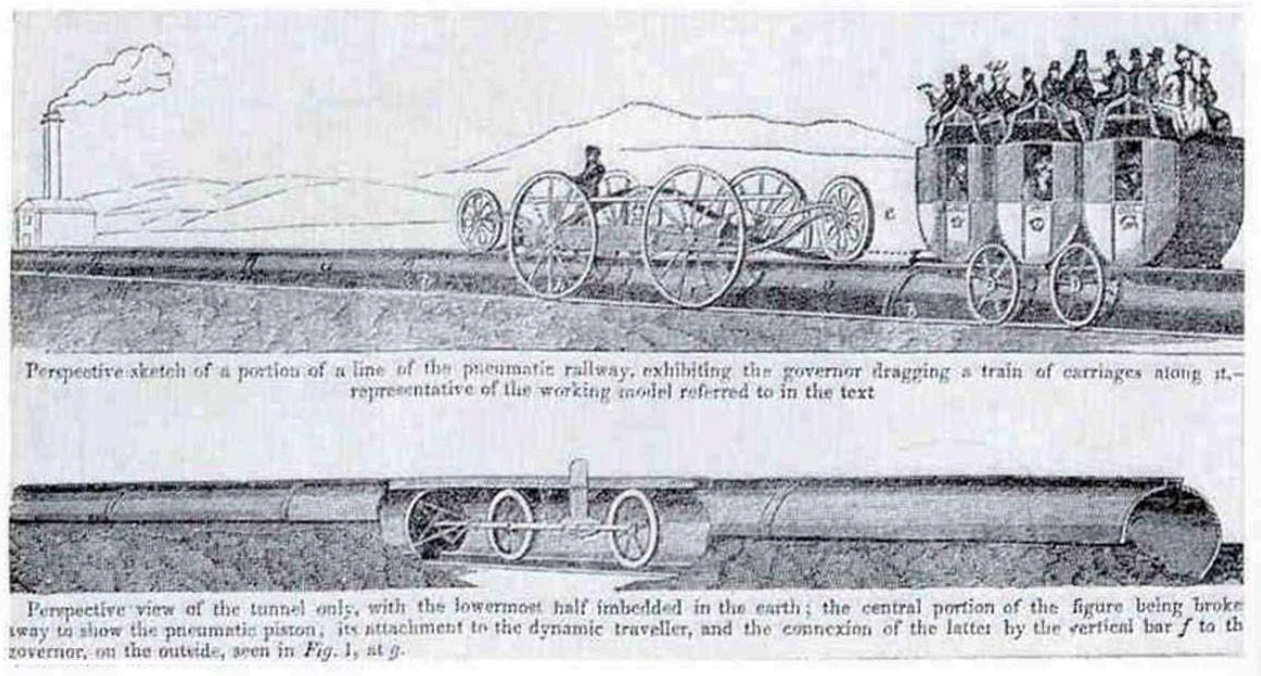

Le projet de chemin de fer pneumatique de Pinkus. En haut, le « gouverneur » tire un véhicule dont les roues reposent sur des rails intégrés au tube; à gauche, le bâtiment de la machine fixe avec sa cheminée; en bas, le piston ou « dynamic traveller » à l'intérieur du tube. Reproduit dans HADFIELD, Charles. ATMOSPHeRIC RAILWAYS, A Victorian ADVENTURE IN SILENT SPEed, 1967, p. 17. Repro Paul Smith, 2008.

(c) Paul Smith.

Sur toute la longueur de la partie supérieure de ce tube, une fente large de deux pouces $(5 \mathrm{~cm})$, surmontée d'une rigole faisant corps avec le tube, permettrait le passage d'un bras métallique vertical qui relierait le piston, que Pinkus baptise le «dynamic traveller » - le voyageur dynamique -, au "gouverneur », c'est-à-dire à la première voiture d'un train. Cette rainure longitudinale serait fermée par «une corde valvulaire » de quatre pouces de diamètre (environ $10 \mathrm{~cm}$ ), fabriquée par longueurs de cinquante yards $(45 \mathrm{~m})$. Il décrit en fait un câble lourd et flexible, fabriqué en fil de fer gainé de feutre et de chanvre et d'une couche de caoutchouc, le feutre préalablement bouilli dans de l'huile de lin ou toute autre matière onctueuse pour le rendre imperméable (fig. $\mathbf{n}^{\circ} \mathbf{1 0}$ ). Soulevé par le passage d'une roue attachée à la voiture, ce câble retomberait ensuite dans la rainure, une roulette aidant la pression atmosphérique à fermer la soupape de manière étanche (fig. $\mathbf{n}^{\circ} \mathbf{1 1}^{11}$. Dans ce système, les machines fixes pour créer le vide seraient installées tous les cinq ou dix milles ( 8 ou $16 \mathrm{~km})$. 
Figure 10

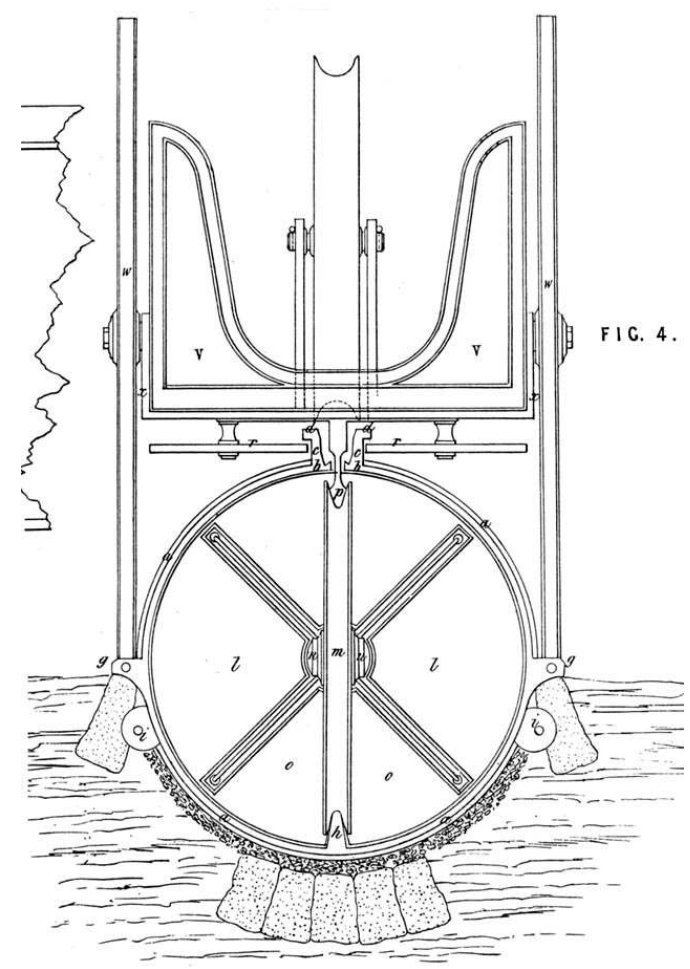

Détail de la planche qui accompagne les spécifications pour le brevet de Pinkus, en date du 30 août 1834, montrant une coupe sur le tube de propulsion surmonté du gouverneur, les rails $(g)$ intégrés au tube. J. G. James collection, Institution of Civil Engineers, London.

(C) Institution of Civil Engineers. 


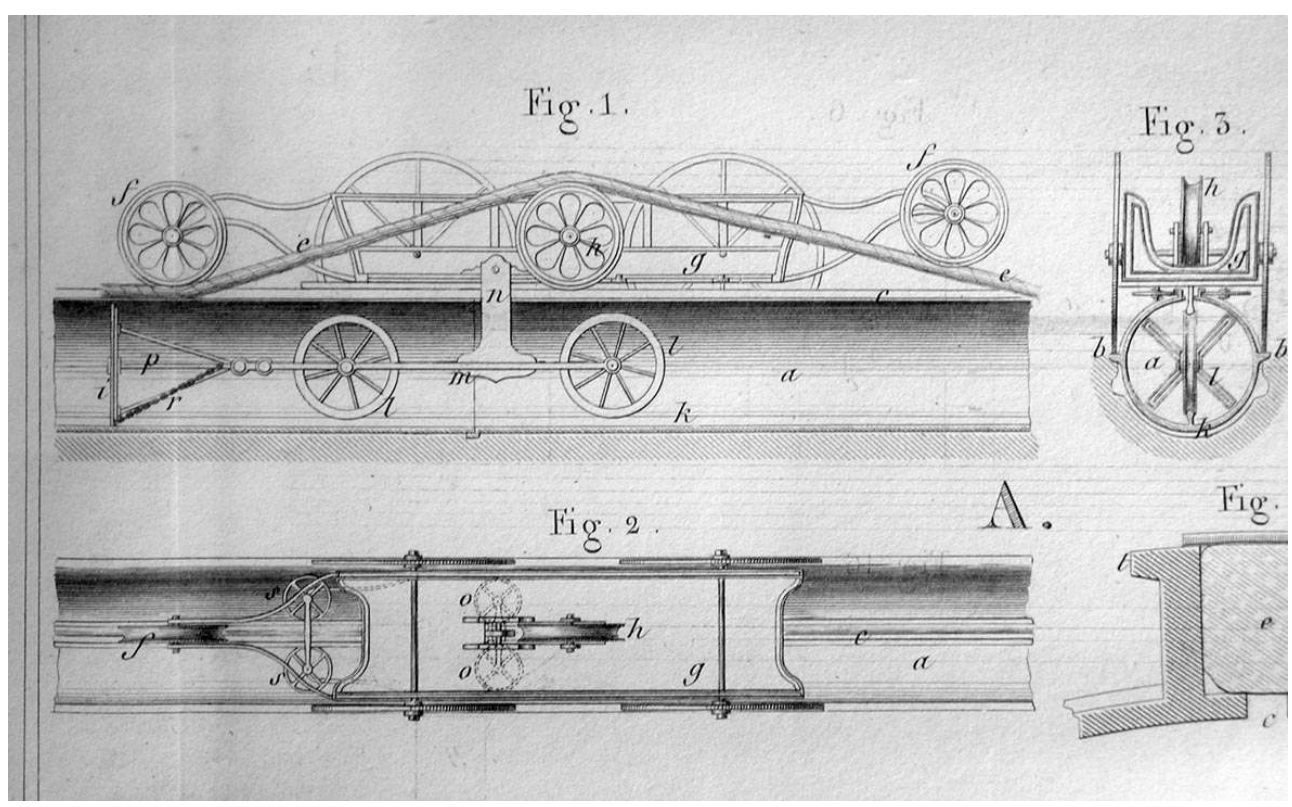

L'invention de Pinkus de 1834, présentée par le BULLETIN DE LA SOciétÉ d'ENCOURAgement POUR L'INDUSTRIE NATIONALE, 1843, planche 911. (Figure 1) : Section longitudinale du tube et du véhicule placé à l'extérieur. (Figure 2) : Le même tube vu en plan, montrant la rainure (c) que vient boucher le câble flexible (e), appuyé par la roulette $(f)$

(c) Société d'Encouragement pour l'Industrie nationale, Paris.

21 En 1839, Pinkus, laissant tomber sa corde, proposa un autre type de fermeture de la soupape, comportant deux bandes métalliques inclinées, composées de plaques de fer et de cuivre, et s'appuyant l'une contre l'autre au-dessus de la fente, ces bandes étant écartées par le passage du bras attaché au piston puis se refermant derrière par leur propre ressort. Il fonda la National Pneumatic Railway Association avec un capital social annoncé de $£ 200000$ mais, pas plus que celle de Vallance, cette compagnie ne semble avoir trouvé d'investisseurs sérieux.

\section{Samuel Clegg et les frères Samuda}




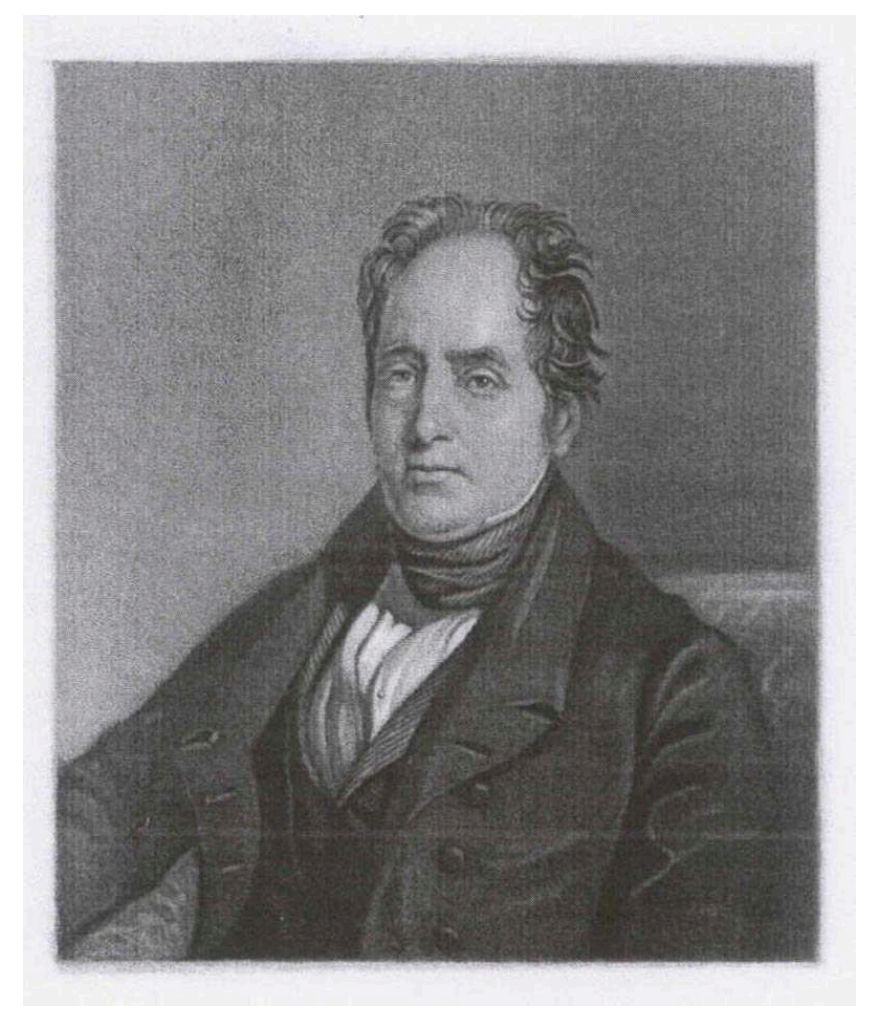

Samuel Clegg, ingénieur civil, portrait en frontispice du Memoir of Mr. Samuel Clegg. QUARTERLY PAPERS ON ENgINEERINg, 1844.

(C) Institution of Civil Engineers.

C'est surtout à partir de ces projets de Pinkus - il y eut d'ailleurs un litige de plusieurs années quant à la priorité de l'invention - que trois autres ingénieurs, aux reins industriels plus solides, purent développer un système pratique qui devint opérationnel, basé sur un premier brevet pris en Angleterre le 3 janvier 1838 et en France le 29 septembre de la même année ${ }^{35}$. Il s'agit de Samuel Clegg (fig. $\left.\mathbf{n}^{\circ} \mathbf{1 2}\right)$ et des frères Jacob et Joseph Samuda. Le premier, né à Manchester en $1781^{36}$, était entré à la manufacture de Boulton et Watt à Soho, où il travailla sous la direction de William Murdoch à ses essais d'éclairage au gaz. Quittant Soho en 1805, Clegg s'occupa par la suite de l'installation de l'éclairage au gaz dans plusieurs filatures du nord de l'Angleterre, ainsi que dans un collège catholique à Stonyhurst dans le Lancashire, avant de rejoindre en 1813 la London and Westminster Chartered Gas-Light and Coke Company, fondée en 1809. En 1815, il prit un brevet pour un compteur à gaz ${ }^{37}$, dont le fabricant, Crosley, aurait fait fortune. Clegg, à un moment donné, se serait associé avec une entreprise de construction mécanique à Liverpool dont la faillite lui coûta sa propre fortune. D'après Hadfield ${ }^{38}$, cet échec fut suivi d'une période où Clegg travailla comme ingénieur pour le compte du gouvernement portugais, ce qui pourrait expliquer son association, lors de son retour en Angleterre, avec les frères Samuda ${ }^{39}$. Appartenant à une famille d'origine portugaise, active de longue date dans les affaires de la communauté juive de Londres, Jacob Samuda (1811-1844) et Joseph d'Aguilar Samuda (1813-1885) étaient les fils d'Abraham Samuda et de Joy d'Aguilar ${ }^{40}$, le premier engagé dans le négoce des compagnies des Indes. Après un apprentissage auprès de l'ingénieur John Hague, connu pour plusieurs brevets mettant la force pneumatique au service de grues et de marteaux de forge, Jacob Samuda s'associa avec son frère cadet pour fonder 
en 1832 une entreprise de construction mécanique à Southwark, sur la rive sud de la Tamise à Londres, entreprise qui se spécialisa par la suite dans la construction navale. En juillet 1843, Jacob, apparemment le plus talentueux des deux frères sur le plan de l'invention mécanique, prit un brevet pour des perfectionnements dans la construction de machines à vapeur applicables à la navigation ${ }^{41}$ mais, le 12 novembre 1844 , il trouva la mort, avec six autres personnes, dans l'explosion de cette machine à bord du Gypsy Queen, bateau en fer à aubes, de $60 \mathrm{~m}$ de longueur, que venaient de lancer de nouveaux chantiers établis à Blackwall ${ }^{42}$.

Figure 13
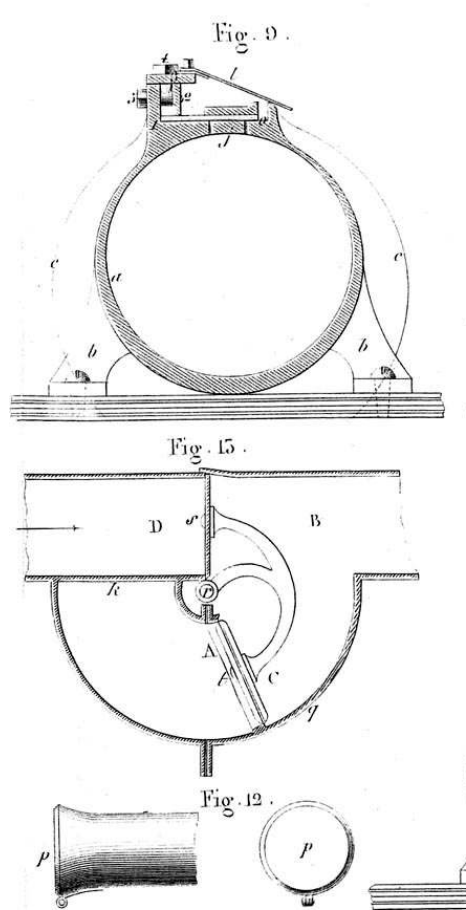
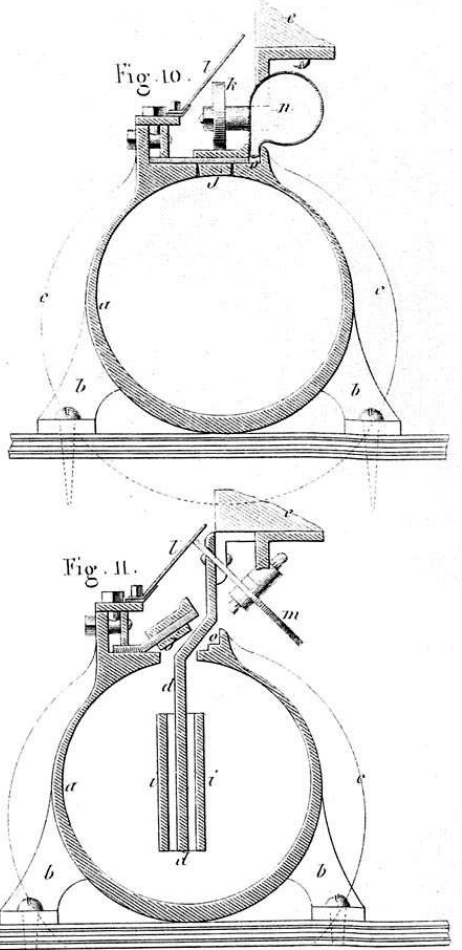

La soupape longitudinale de Clegg et Samuda présentée par le BULLETIN DE LA SOCIÉTÉ

D'ENCOURAgEMENT POUR L'INDUSTRIE NATIONALE, 1843, planche 911. (Figure 9) : Section transversale du tube de propulsion avant l'arrivée du piston voyageur, avec le clapet en cuir fermé. (Figure 10) : Section transversale du tube avec la soupape fermée immédiatement après le passage de la tige de propulsion. (Figure 11) : Section transversale du tube au moment du passage de la tige. (Figure 12) : Vue de profil et de face de la soupape de sortie du tube, composée d'un simple couvercle à charnière en bois garni de cuir. (Figure 13) : Section horizontale (le couvercle enlevé) de la boîte renfermant la soupape d'entrée du tube.

(c) Société d'Encouragement pour l'Industrie nationale, Paris.

Dans l'invention brevetée par Samuel Clegg et Jacob Samuda, perfectionnée par de nouveaux brevets en 1844 et 1845 , le tube - le " conduit agissant », le " tuyau continu» ou le «tube de propulsion » - était fermé à ses extrémités par des soupapes d'entrée et de sortie (fig. $\mathbf{n}^{\circ} \mathbf{1 3}$ ). La fente longitudinale d'environ un pouce et demi de largeur $(3,81 \mathrm{~cm})$ était recouverte par une lame de cuir de bœuf de première qualité, large de cinq pouces $(12,70 \mathrm{~cm})$ et formant une espèce de clapet, renforcé en dessus et en dessous par une série de plaques de fer, comme autant d'écailles. Un bord de cette «lanière-soupape » était boulonné à l'un des deux côtés de la fente pour faire une charnière continue. L'autre bord reposait sur la lèvre opposée de la fente, où une petite 
gouttière contenait un mastic composé pour un quart de cire d'abeilles et pour trois quarts de suif, composition solide à la température de l'atmosphère mais devenant liquide avec un léger réchauffement. D'après Jacob Samuda, présentant l'invention devant l'Institution of Civil Engineers en mai 1844, l'idée de cette composition, servant de soudure, revenait à Samuel Clegg ${ }^{43}$. Longue d'environ quatre pieds $(1,2 \mathrm{~m})$ et d'un demi-pouce d'épaisseur $(1,27 \mathrm{~cm})$, la barre de connexion reliant le châssis du piston et le « chariot remorqueur " $^{44}$ était coudée à 45 degrés pour éviter l'ouverture trop grande du clapet, soulevé de seulement un pouce et demi $(3,8 \mathrm{~cm})$. Laissant rentrer librement l'air atmosphérique derrière le piston, cette ouverture était effectuée par quatre ou cinq galets attachés au châssis à l'intérieur du tube. Ce châssis avait six mètres de longueur, une boule en fonte à l'arrière servant de contrepoids au piston, lui-même simple rouleau de fonte armé à ses deux extrémités d'une mâchoire pinçant une lame de cuir (fig. $\mathbf{n}^{\circ}$ 14). L'ensemble de la soupape était protégé contre les intempéries par un couvercle de cuir soulevé par le même appareil que la soupape. Attachée à l'arrière du wagon directeur, une roue en acier appuyait sur la lanière de cuir pour refermer la soupape. Cette roue était suivie d'un appareil appelé «le chauffoir ", comportant une lame de cuivre longue d'environ cinq pieds $(1,50 \mathrm{~m})$ et remplie de charbon de bois incandescent. Celui-ci liquéfiait le mastic, scellant de nouveau le joint et assurant l'herméticité de la fermeture, en attendant le passage du train suivant (fig. $\mathbf{n}^{\circ} \mathbf{1 5}$ ).

Figure 14

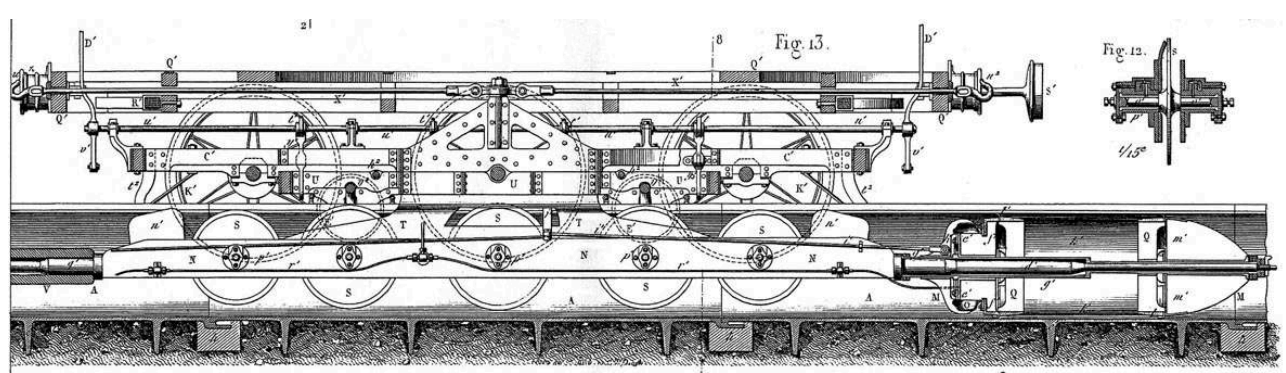

Chemin de fer atmosphérique de Saint-Germain, coupe sur le wagon directeur attaché au châssis du piston dans le tube (le piston à droite). Armengaud Aîné, Chemins de fer atmosphériques.

PUBLICATION INDUSTRIELle DES MACHINES, OUTILS et APPAREILS LES PLUS PERFECTIONNÉS ET LES PLUS RÉCENTS EMPLOYÉS DANS LES DIFFÉRENTES BRANCHES DE L'INDUSTRIE FRANÇAISE ET ÉTRANgÈRE, 1848. Planche 9 , figure 13.

(c) Société d'Encouragement pour l'Industrie nationale, Paris. 


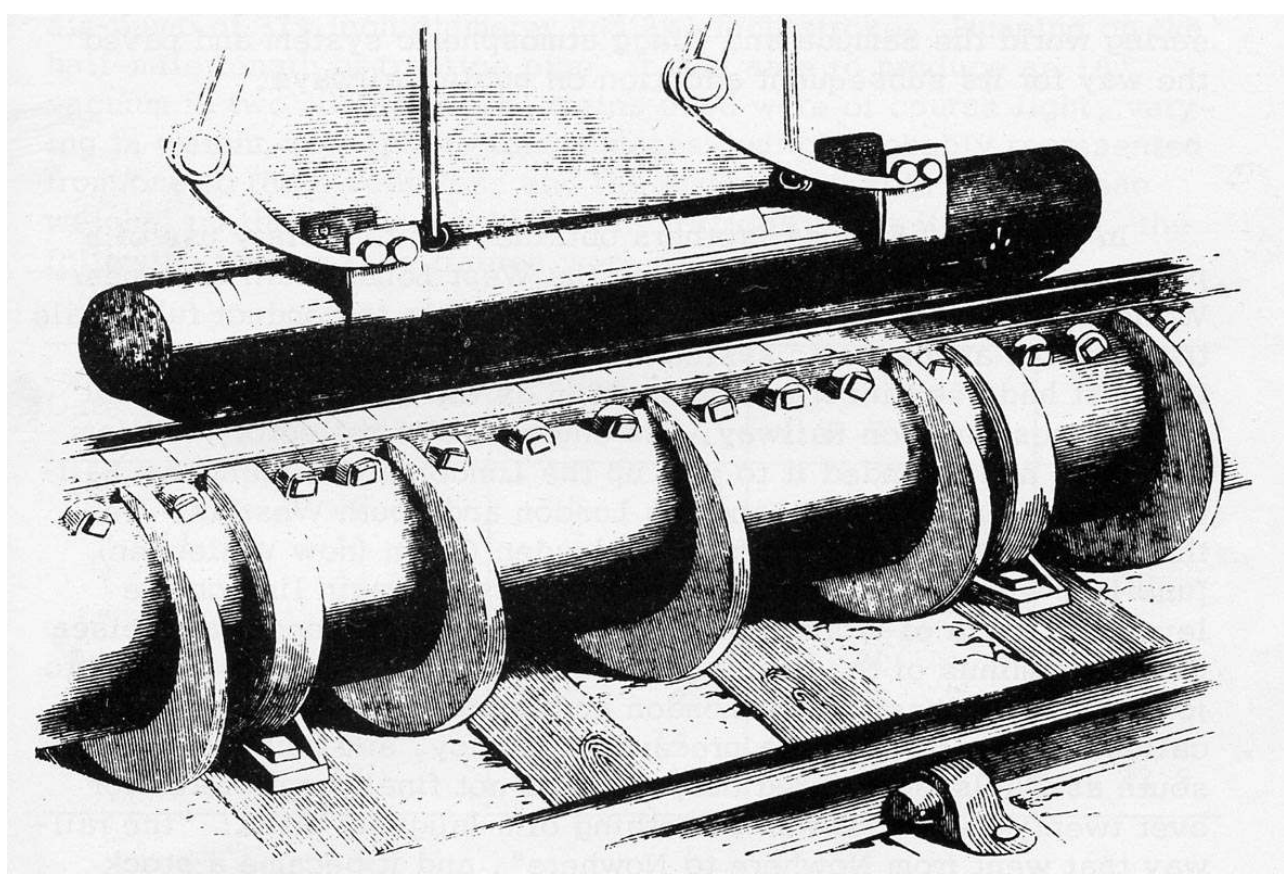

Le « chauffoir » destiné à faire fondre le mastic pour sceller la fermeture de la soupape longitudinale. Reproduit par CLAYTON, Howard. The Atmospheric Railways, 1967, p. 23. Repro Paul Smith, 2008. (C) Paul Smith.

Les premiers essais de cet appareillage, à une échelle réduite, furent entrepris au cours de l'année 1838 à Paris, où Clegg était déjà en liaison avec des industriels du gaz ${ }^{45}$. Quelques années plus tard, l'ingénieur Edmond Teisserenc se souvint de ce premier chemin d'essai construit en France: «Le petit nombre d'ingénieurs et de savans français qui s'occupaient de chemins de fer il y a sept ans, se rappellent sans doute qu'à cette époque, un petit chemin d'essai, conçu d'après un nouveau système, fut construit à Chaillot et fut examiné par diverses commissions. L'invention était alors dans son enfance, et si quelques esprits pénétrans furent frappés de ce qu'elle renfermait d'ingénieux, la grande masse du public n'y vit qu'un joujou d'enfans. Les inventeurs ne se découragèrent pas. Loin d'abandonner leur idée, ils la poursuivirent...46 ${ }^{46}$ Ces essais eurent donc lieu à la célèbre fonderie de Chaillot dans l'actuel seizième arrondissement de Paris. Créé par les frères Jacques-Constantin et Augustin-Charles Périer, cet établissement avait été l'un des premiers lieux d'importation des machines de Boulton et Watt, notamment pour la pompe à feu de Chaillot de la Compagnie des Eaux de Paris, fondée par les deux frères en août $1778^{47}$. En 1818, la fonderie, devenue l'un des premiers fournisseurs français de machines à vapeur ${ }^{48}$, était passée entre les mains de la Compagnie Périer-Edwards-Chaper qui, depuis 1836, s'était lancée dans la construction de locomotives à vapeur ${ }^{49}$. Dans cette compagnie, Edwards était Humphrey Edwards, l'un des nombreux ingénieurs et constructeurs mécaniques venus d'Angleterre pour s'établir à Paris au début de la Restauration. Il meurt en 1829, son fils Henry-Hind Edwards prenant sa succession. C'est peut-être cette connexion britannique qui explique comment les ateliers de Chaillot - qui employaient bon nombre d'ouvriers anglais - aient pu être utilisés en 1838 pour l'expérimentation, conduite sous la direction de celui qui semble avoir été leur principal agent à Paris James Bonfil, d'un « échantillon » du système de Clegg et Samuda. 
Les «nouveaux chemins de fer de M. Clegg " firent l'objet d'une présentation à l'Académie des Sciences dans sa séance du 18 février 1839. Le mémoire résume les nombreux avantages potentiels du système atmosphérique: légèreté du wagon conducteur permettant des économies dans la construction du chemin, suppression de la dépense excessive qu'exige la réparation des locomotives, suppression de tout danger d'explosion, impossibilité que les convois sortent des rails, possibilité de parcourir des courbes de rayons assez courts, suppression des secousses désagréables qui, dans les locomotives ordinaires, résultent des coups brusques de la bielle de la machine, etc. Il remarque, en conclusion, «qu'il existe déjà à Paris un échantillon du chemin pneumatique qui a fonctionné devant un grand nombre de personnes ${ }^{50} »$. Par la suite, James Bonfil aurait réalisé des essais du système au Havre, dans les ateliers des frères Nillus ${ }^{51}$, tandis que d'autres essais, décrits par Clegg dans une brochure publiée au printemps de l'année $1839^{52}$, furent entrepris aux ateliers des frères Samuda à Southwark. Utilisant un tube expérimental d'environ trois pouces et demi de diamètre $(9 \mathrm{~cm})$, long de trente pieds $(9 \mathrm{~m})$ et dans lequel le vide fut d'abord créé par des pompes actionnées à la main par quatre hommes, ces essais, employant ensuite une machine à vapeur et un tube de trente puis cinquante mètres de long, permirent de tracter une voiture portant deux hommes. Dans son numéro du 11 mai 1839, le Mechanics' Magazine rendit également compte de ces expériences, réalisées devant de nombreux membres du Parlement, directeurs de compagnies ferroviaires et ingénieurs. La machinerie fonctionna à merveille et donna pleine satisfaction. Pour le Magazine, les avantages du nouveau système - économie, sécurité, vitesse et absence de tout risque d'explosion étaient très évidents, contrastant fortement avec les inconvénients du système à locomotives. À partir de ces premiers essais à Southwark, Clegg put déjà tenter une estimation des économies qu'apporterait son système atmosphérique sur une grande ligne : l'installation coûterait $£ 10456$ par mille, comparé à $£ 42000$ par mille pour le système à locomotives (locomotives comprises) et l'exploitation reviendrait à $£ 1246$ par an et par mille, comparé à $€ 4141$ pour le système à locomotives, d'après les données de la ligne Liverpool-Manchester.

\section{Clegg et les Samuda à Wormwood Scrubs}

Clegg et Samuda conclurent ensuite un accord avec la Compagnie du Birmingham, Bristol \& Thames Junction Railway - accord apparemment négocié par James Bonfil ${ }^{53}$ leur permettant d'entreprendre des essais à plus grande échelle sur un tronçon de voie non encore construit, situé dans l'ouest de Londres, à Wormwood Scrubs. Dorénavant de neuf pouces de diamètre intérieur $(22,86 \mathrm{~cm})$, un tube d'un demi-mille de longueur $(805 \mathrm{~m})$ fut installé entre des rails de récupération, le vide généré par un appareil pneumatique mû par une machine de seize chevaux-vapeur, récupérée d'un des bateaux des Samuda. Commencés le 11 juin 1840, ces essais se poursuivirent régulièrement jusqu'en février 1841 et ensuite, par intermittence ou à la demande de certains visiteurs, jusqu'en mars 1843. Le public était admis, gratuitement, deux jours par semaine. Les essais connurent un succès retentissant, dépassant le seul monde ferroviaire. Des vitesses remarquables de 45 milles à l'heure $(67 \mathrm{~km} / \mathrm{h})$ furent atteintes ${ }^{54}$ et des trains tractés avec 75 passagers, pesant plus de onze tonnes. La composition d'étanchéité fonctionnait parfaitement bien, été comme hiver. Wormwood Scrubs (nom synonyme aujourd'hui de la prison qui s'y trouve) devint un lieu de sortie 
"fashionable», attirant ingénieurs et investisseurs potentiels mais aussi d'autres curieux parmi lesquels le prince Albert, Charles Babbage et son amie Ada Lovelace (la fille de Lord Byron), Charles Manby, l'astronome royal George Airy ${ }^{55}$, l'ambassadeur français M. de Sainte-Aulaire ${ }^{56}$...

En France, ces essais londoniens provoquèrent une certaine curiosité. E. Hérard, élèveingénieur des Ponts et Chaussées, visita le chemin d'essai en 1843, malheureusement après la fin des expériences. Mais « tous les ingénieurs anglais auprès desquels [il avait été] introduit et qui avaient suivi les expériences de Wormwood Scrubs faisaient un rapport très-favorable du système atmosphérique, et lui croyaient réellement un avenir $^{57}$. » Plus officiellement, et vraisemblablement à la suggestion de l'ambassadeur français à Londres, un spécialiste des chemins de fer, Pierre-Edmond Teisserenc de Bort, fut envoyé en Angleterre par le ministre des Travaux publics. Présent à Londres au mois d'octobre $1842^{58}$, trop tard pour être témoin des premières expériences, il trouva le chemin d'essai de Wormwood Scrubs dans «un état inexprimable de délabrement [...] dans lequel la voie fatiguée par les pluies n'a plus une inclinaison régulière, dans lequel le parallélisme des rails et du tube est complètement détruit, le tube a pris une position sinueuse et tourmentée, etc. »

Figure 16

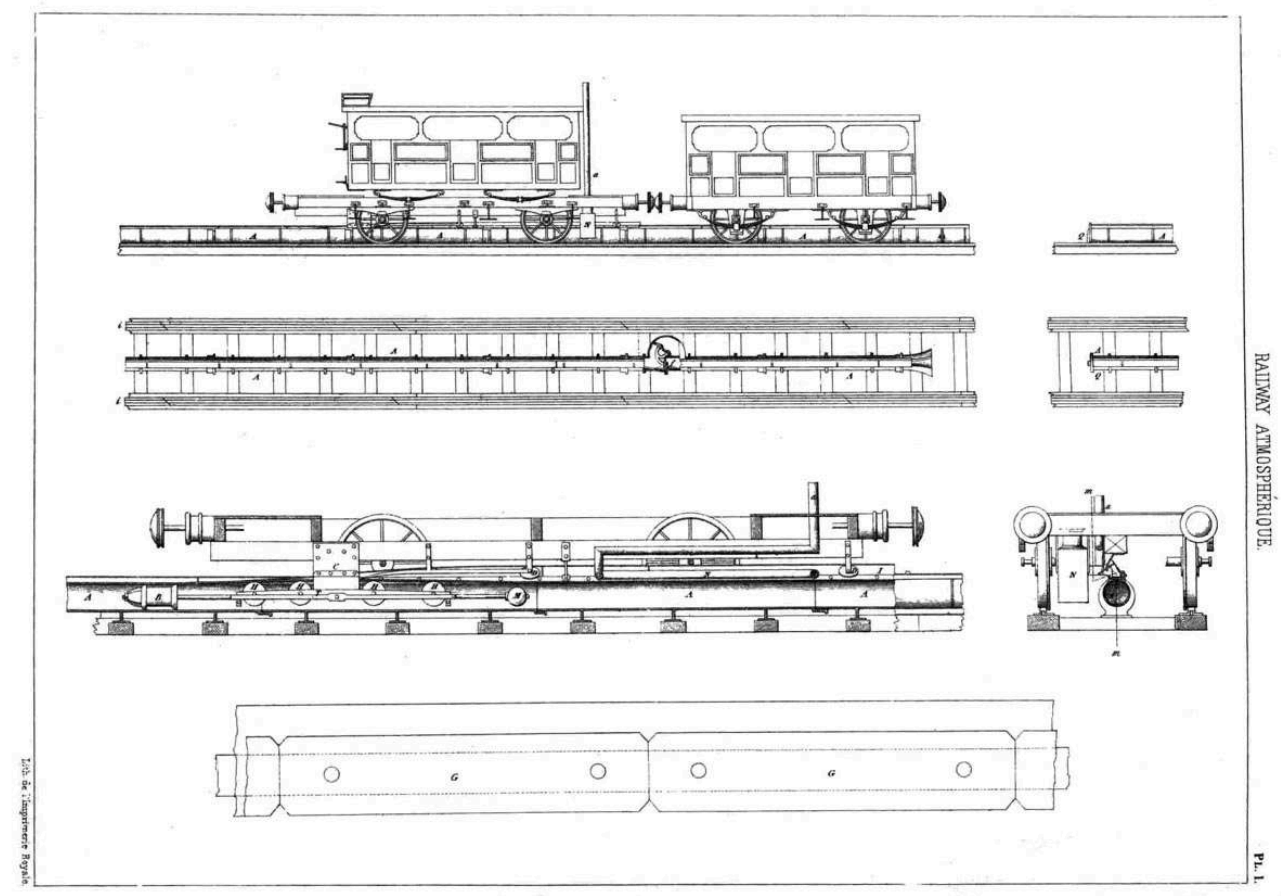

Planche relative au chemin de fer atmosphérique établi à Wormwood Scrubs, représentant l'ensemble de l'appareil en plan et en élévation, avec coupes longitudinales et transversales du tube et du chariot de tête. TEISSERENC, Edmond. RAPPORT ADRESSÉ À M. LE MINISTRE DES TRAVAUX PUblics SUR LES CHEMINS DE FER, 1843. Planche 1.

(C) Centre de ressources documentaires, administratives et juridiques du ministère de l'Écologie, de l'Energie, du Développement durable et de l'Aménagement du Territoire.

Ce qui n'empêcha pas son rapport, daté du 19 mars 1843, d'être franchement dithyrambique, reprochant même aux inventeurs et promoteurs les plus convaincus du système atmosphérique de ne pas avoir bien saisi le véritable caractère de l'invention 
qu'ils avaient entre les mains, "encore une révolution dans l'histoire des chemins de fer ${ }^{59}$ » (fig. $\mathbf{n}^{\circ}$ 16).

Pour Teisserenc, le nouveau système atmosphérique était manifestement supérieur à la traction par câble, qui imposait des tracés rectilignes et qui nécessitait une grosse dépense d'énergie pour faire tourner les immenses tambours servant à enrouler les cordes d'un poids considérable : 80 tonnes par exemple sur la ligne de Blackwall, à Londres. L'opération de ce système requérait également de grandes précautions pour éviter tout changement brusque de vitesse qui romprait le câble. Mais Teisserenc était également convaincu de la supériorité du système atmosphérique sur les chemins de fer ordinaires. Celle-ci tenait en quatre considérations principales. Tout d'abord, les dépenses de première exécution seraient moindres: toute collision étant impossible, les chemins pourraient être exécutés à simple voie. La hauteur des ponts au-dessus du chemin pourrait être moins élevée, n'ayant pas à prendre en compte la cheminée des locomotives. Le matériel des voitures et des wagons, n'ayant ni chocs et ni secousses à redouter, pourrait être construit plus légèrement, admettant de grandes vitesses même sur des pentes et des courbes. Le chemin atmosphérique pourrait épouser les contours naturels du terrain, supprimant terrassements et travaux d'art tels que viaducs et tunnels. En France, selon Teisserenc, le chemin pourrait même suivre l'un des accotements des routes royales, évitant ainsi de coûteuses expropriations et ne troublant pas des « existences acquises ». Deuxièmement, en ce qui concerne les frais d'exploitation, malgré la surveillance que nécessiterait l'état du tube et le renouvellement périodique de la composition assurant sa fermeture hermétique (composition ne coûtant que 2,50 F le kilogramme), la voie serait moins fatiguée et demanderait moins de réparations. Avec des machines fixes, les frais quotidiens de locomotion (houille, graissage, salaires des chauffeurs et mécaniciens...) seraient inférieurs à ceux des locomotives ordinaires. Troisièmement, en ce qui concerne la vitesse, celle du système atmosphérique pourrait être augmentée par le simple agrandissement du diamètre du cylindre de la pompe pneumatique tandis qu'avec les locomotives ordinaires, des vitesses de 60 à $70 \mathrm{~km}$ à l'heure ne pouvaient pas être utilement dépassées. Teisserenc note aussi le silence des trains atmosphériques, avantage appréciable «quand on se trouve à proximité d'une grande voie publique fréquentée par des animaux de trait et par des bestiaux ». Enfin, supériorité majeure et décisive : la sécurité. «Avec l'appareil atmosphérique pas de collisions, pas d'incendies, pas de rupture d'essieu... » Rappelons ici que ce rapport de Teisserenc date de moins d'un an après la catastrophe de Meudon, à laquelle il fait allusion, ainsi qu'à d'autres accidents arrivés sur le chemin de fer de Liège et sur celui de Tsarkoé-Selo à SaintPétersbourg.

Le Journal des Chemins de fer, premier journal ferroviaire spécialisé en France, lancé en janvier 1842 et dirigé par l'Anglais F. R. Whitelock, suivait également les essais entrepris à Wormwood Scrubs, constatant dans son numéro 6, du 15 avril 1842, « que la question de savoir si des trains de voitures peuvent être mis en mouvement au moyen de la pression atmosphérique n'est donc plus douteuse » et notant, le 18 juin, que «le principe atmosphérique a fait rapidement son chemin dans l'opinion publique et plusieurs ingénieurs distingués de la Grande-Bretagne et de l'Irlande paraissent très disposés à l'adopter. » Le Journal rendait compte de la conférence du professeur Charles Vignoles ${ }^{60}$, illustrée par des maquettes et de grands dessins pour expliquer l'application du principe pneumatique à la locomotion lors du congrès annuel de la British 
Association for the Advancement of Science, tenu à Manchester en juin 1842. La conférence passait en revue les travaux de Papin, Medhurst, Vallance et Pinkus pour arriver à la soupape de Clegg, invention ingénieuse et remarquable, « caractéristique de l'âge présent ». En octobre de la même année, Vignoles présenta de nouveau le système atmosphérique devant la Royal Cornwall Polytechnic Society. Comparant le système, encore dans son enfance, à celui de la traction par câbles, Vignoles le décrivit comme "a rope of air ", une corde d'air, permettant d'utiliser tout l'effet dynamique de la puissance des machines fixes. «Le système atmosphérique, dit Monsieur Vignoles, fait une profonde impression sur tous les savants de l'Europe ${ }^{61}$."

31 Autre organe gagné par l'enthousiasme atmosphérique naissant, L'Illustration, lancée en mars 1843 par le saint-simonien Édouard Charton. Dans son numéro du 26 août 1843, s'inspirant du rapport de Teisserenc pour en détailler les avantages, la revue présenta le nouveau système de locomotion: "Nous avouons que nous craignons qu'on ne nous accuse d'engouement pour la chose nouvelle si nous disons qu'il nous paraît supérieur au système des locomotives sous le triple point de vue des dépenses de construction et d'exploitation, de la vitesse et de la sécurité. » L'Illustration n'hésita pas à porter à $50 \%$ l'économie générale du nouveau système par rapport à l'ancien et invita le gouvernement à concentrer son attention sur les essais en cours de préparation en Irlande.

\section{De Kingstown à Dalkey}

Les démonstrations publiques à Londres avaient fini, en effet, par convaincre une compagnie de chemins de fer, celle de Dublin à Kingstown, d'entreprendre un essai commercial du système de Clegg et Samuda. Teisserenc souligna l'honneur qui revenait ainsi à l'Irlande, l'Angleterre n'ayant pas voulu accueillir l'invention car la plupart de ses ingénieurs étaient intimement intéressés au succès des ateliers dans lesquels se construisaient les locomotives ${ }^{62}$. 


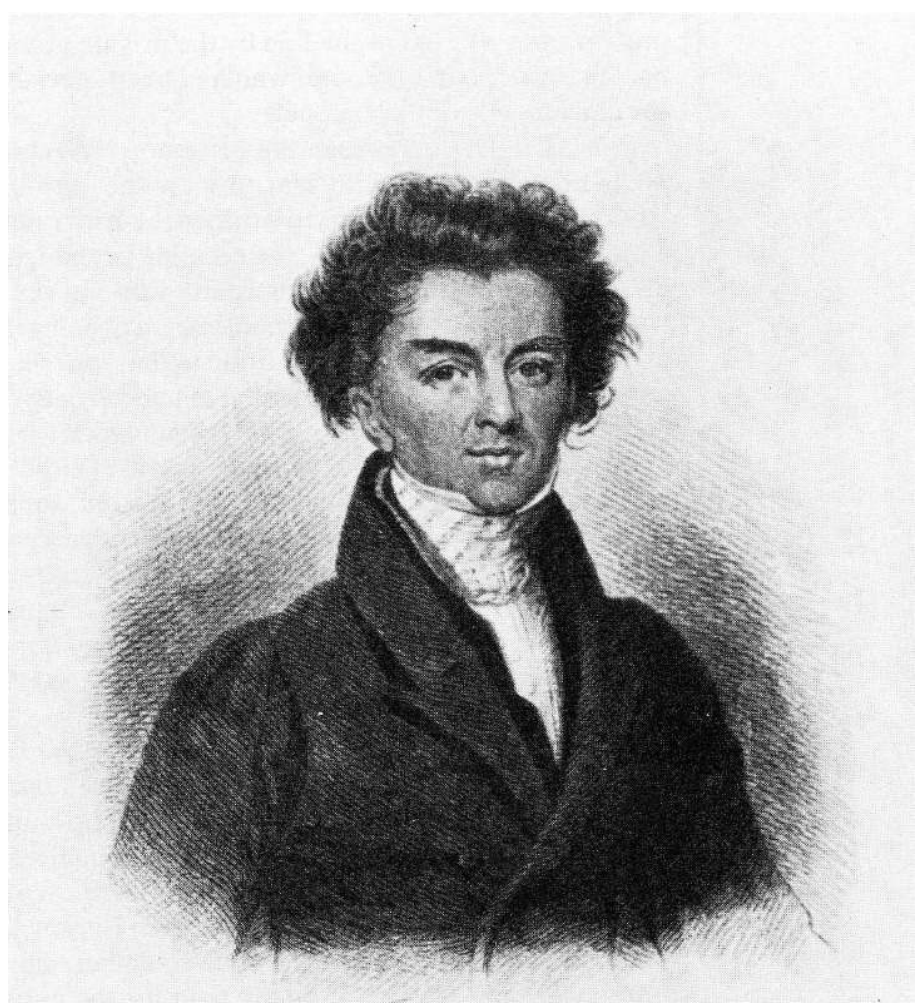

Portrait de Charles Blacker Vignoles gravé par R. Roffe en 1835 pour le Mechanics' Magazine, reproduit par VIGNOLES, K. H. CHARLeS BLACKer VIgNOLES : ROMANTIC ENgINEeR, 1982. p. 49. Repro Paul Smith, 2008.

(C) Paul Smith.

Fondée en novembre 1831 à la suite d'un acte du Parlement du 6 septembre, cette compagnie de Dublin à Kingstown avait inauguré le 17 décembre 1834 une ligne de cinq milles et demi (environ neuf kilomètres) partant de Dublin, de la gare de Westland Row $^{63}$, pour rejoindre vers le sud le port de Kingstown ${ }^{64}$. L'ingénieur de cette ligne était Charles Vignoles (fig. $\mathbf{n}^{\circ} \mathbf{1 7}$ ).

Comme le chemin de fer de Paris à Saint-Germain, ouvert deux ans et demi plus tard, ce tout premier chemin de fer irlandais était essentiellement conçu comme une ligne de promenade et de plaisir, destinée aux «classes supérieures de la société ». Elle desservait une succession de lieux de baignade le long de la baie de Dublin, offrant, autour de Kingstown, des occasions "d'exercices salutaires dans une atmosphère pure et de beaux paysages romantiques ${ }^{65} \%$. Quant à la vue sur la mer, que certains propriétaires récalcitrants ne voulaient pas voir défigurée par le chemin de fer, la compagnie pensait, au contraire, qu'avec ses trains en mouvement rapide, elle gagnerait un premier plan nouveau et nullement dépourvu d'intérêt (fig. $\mathbf{n}^{\circ} \mathbf{1 8}$ ). La compagnie irlandaise entrevoyait également un trafic de marchandises pour sa ligne, notamment dans le transport de charbon importé de Liverpool, avec un fret de retour en granite, dont la demande était constante dans le grand port anglais. Au-dessus de Kingstown, à Dalkey, se trouvaient en effet d'importantes carrières, exploitées à partir de 1817 pour la construction du nouveau port, un double " tramway » (un funiculaire à cheval) servant à descendre les blocs de pierre (fig. $\mathbf{n}^{\circ} \mathbf{1 9}$ ). 
Figure 18

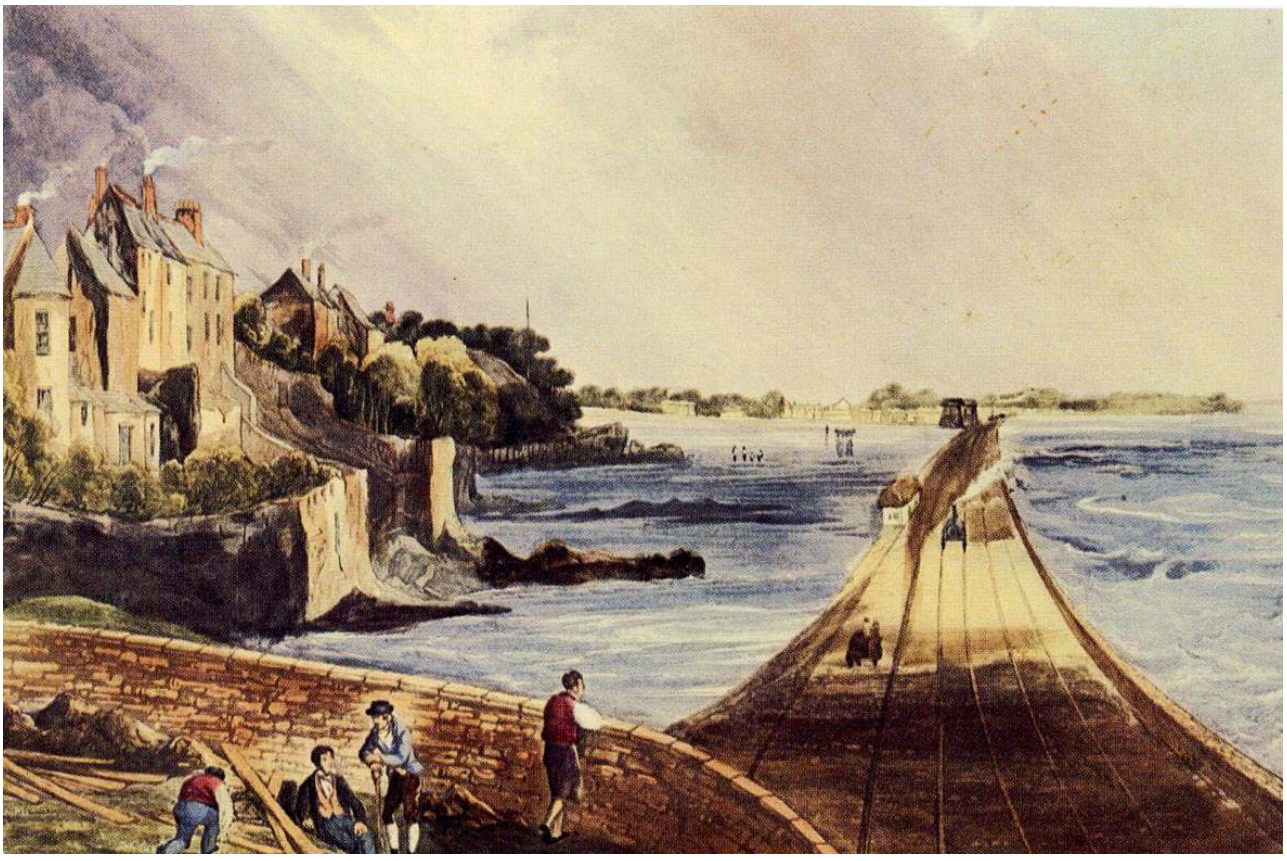

Vue du chemin de fer de Dublin à Kingstown en 1834, avec Dublin dans le lointain. Dessinée par A. Nichol et gravée par J. Harris et reproduite en carte postale publiée par The Exchange Bookshop, Dalkey, 2006. Repro Paul Smith, 2008.

(c) Paul Smith.

\section{Figure 19}

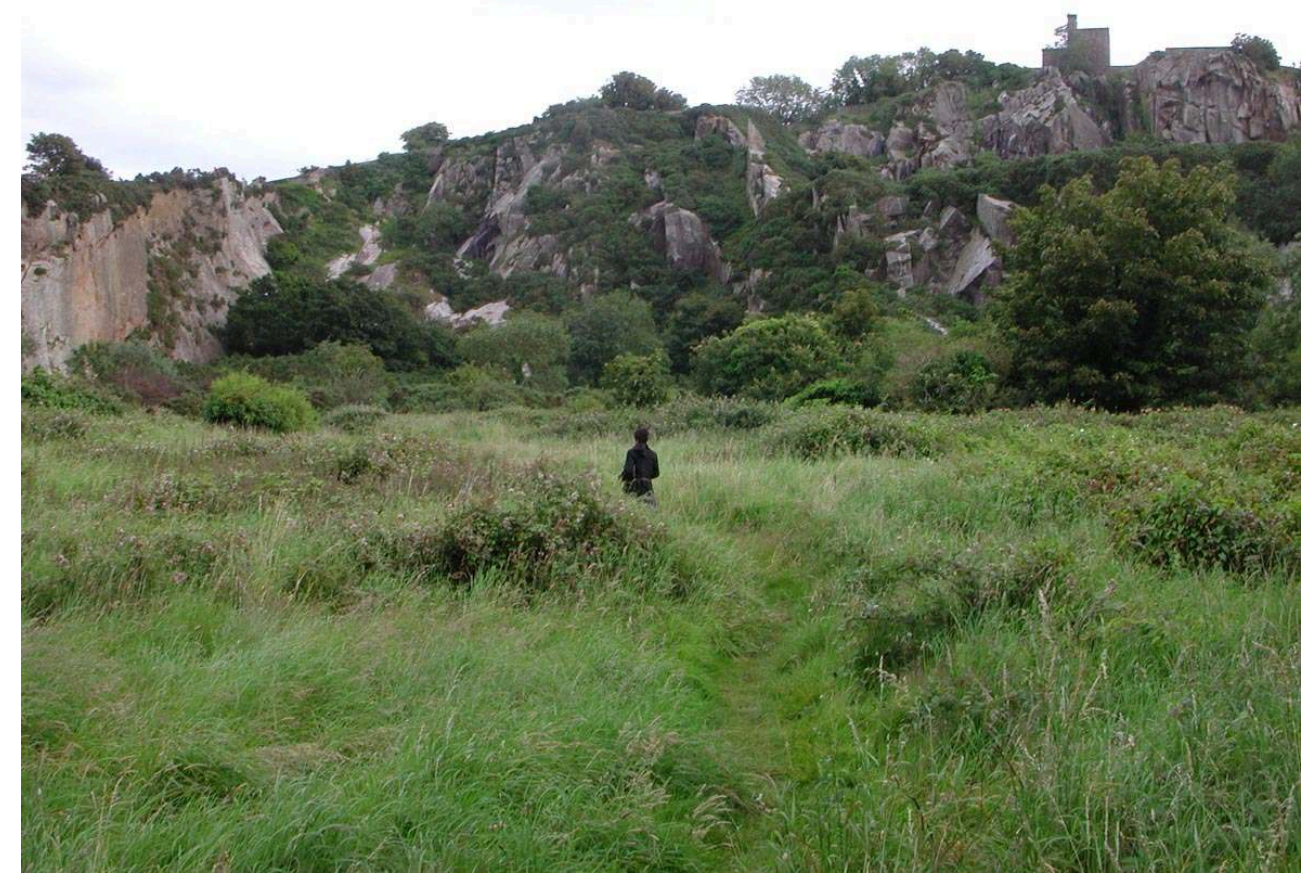

Les anciennes carrières de granit au-dessus de Dalkey. Photo Paul Smith, 2007.

(c) Paul Smith. 
C'est sur ce parcours d'environ un mille et trois quarts (près de trois kilomètres) entre le port de Kingstown et le village de Dalkey, présentant un dénivelé total de 71 pieds (22 m), que la compagnie, dès 1841, envisageait une extension de sa ligne et un essai commercial du système de Clegg et Samuda (fig. $\mathbf{n}^{\circ} \mathbf{2 0}$ ). Vignoles, très enthousiasmé par ce qu'il avait vu à Wormwood Scrubs, avait réussi à convaincre, à son tour, le secrétaire de la compagnie Thomas F. Bergin, ainsi que son trésorier James Pim, membre d'une importante famille de banquiers de Dublin. Tous les deux devinrent d'ardents promoteurs du système atmosphérique. Une brochure de Bergin évoquait la " révolution presque incroyable " à venir dans les transports mécaniques ${ }^{66}$ tandis que deux autres rédigées par Pim, argumentées de manière convaincante et illustrées par des gravures dues à Vignoles ${ }^{67}$ (fig. $\mathbf{n}^{\circ}$ 21), suggéraient que les chemins de fer atmosphériques étaient particulièrement appropriés au contexte irlandais et à son économie rurale. D'une part, étant donné le trafic en voyageurs et en marchandises envisageable sur les chemins de fer irlandais, des chemins à voie unique devraient suffire très amplement. Or, seul le système atmosphérique pouvait garantir ces voies uniques des risques de collision. D'autre part, les machines à vapeur fixes brûleraient du charbon de terre, déjà moins cher que le coke utilisé par les locomotives, ou même de la tourbe. En outre, lorsqu'elles ne serviraient pas à faire le vide dans le tube de propulsion - à peine dix minutes par heure -, ces machines, installées tous les trois milles, seraient disponibles pour d'autres tâches utiles : moudre l'avoine ou le blé, scier bois ou pierre, pomper l'eau pour assécher ou irriguer des terrains agricoles...

Figure 20

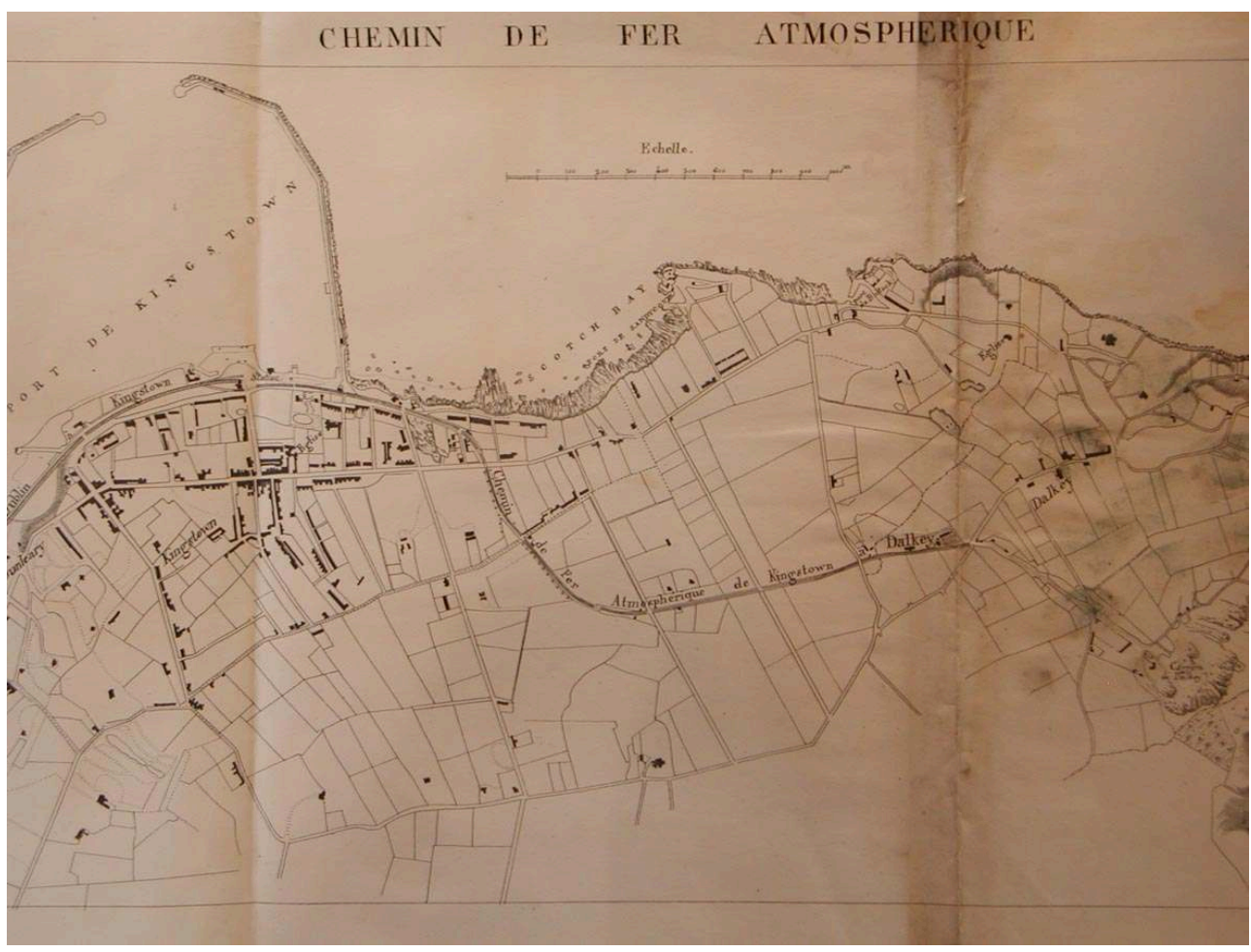

Chemin de fer atmosphérique de Kingstown à Dalkey, plan établi vers 1844, Archives nationales F/ 1411171

(c) Archives nationales. 


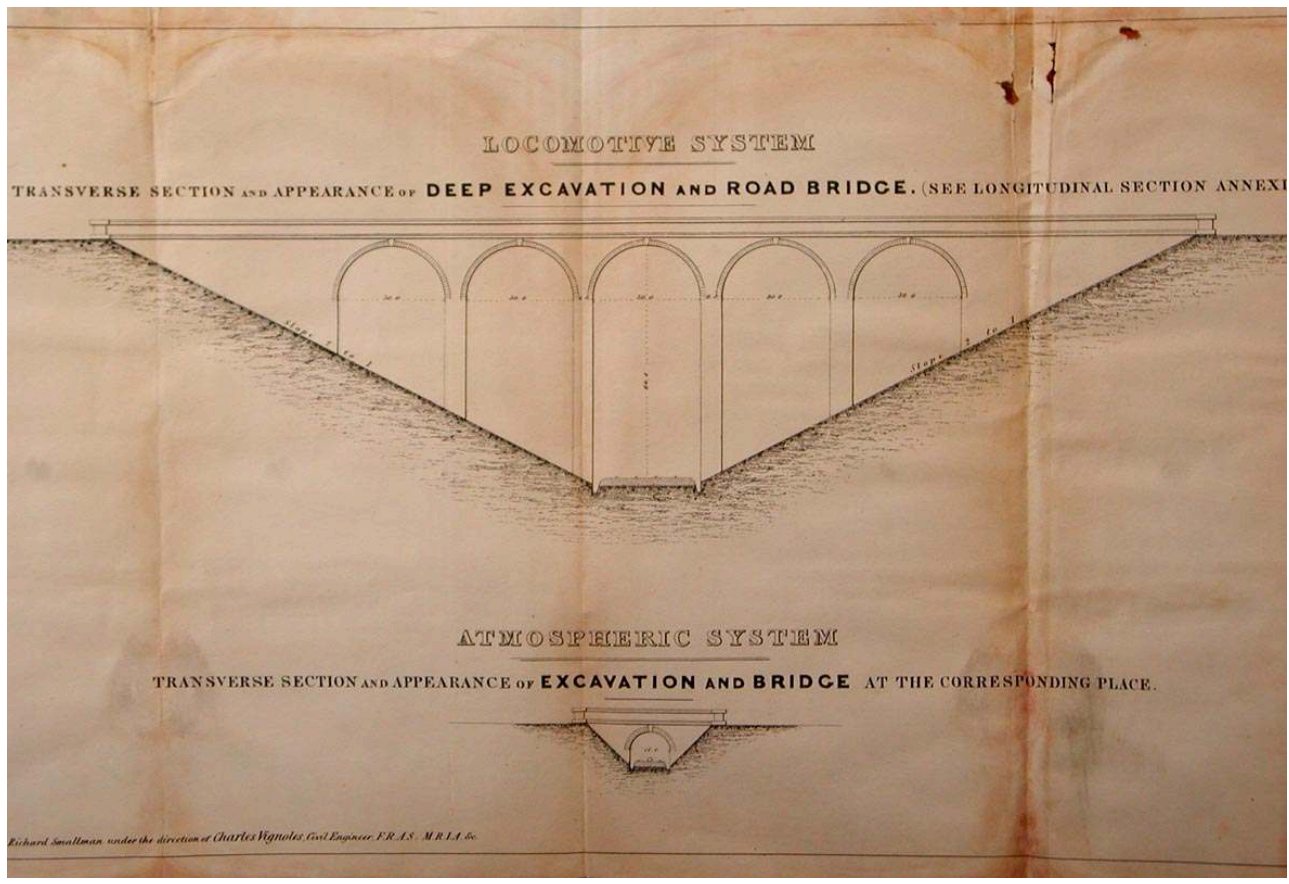

Gravure illustrant les économies en matière d'ouvrages d'art par comparaison entre le système à locomotives, en haut, et le système atmosphérique, en bas. Gravure par Richard Smallman sous la direction de Charles Vignoles, publiée dans PIM, James. THE ATMOSPHERIC RAILWAY, A LETTER TO THE RIgHT HON. THE EARL OF RIPON, PRESIDENT OF THE BOARD OF TRADE, 1842 (Archives nationales, F/14 11171).

(c) Archives nationales.

Quant à Clegg et aux Samuda, on les imagine ravis de cette occasion de faire enfin la preuve de leur invention, une démonstration que celle-ci n'était pas un «jouet ingénieux » mais, au contraire, un engin " d'une puissance prodigieuse d'utilité 68 ». Dès lors, l'accord qu'ils signaient avec la compagnie, approuvé par les actionnaires dans une assemblée générale extraordinaire du 6 avril 1842, comportait des termes très favorables aux intérêts de cette dernière. Pour la somme forfaitaire de $€ 11000$ (environ $275000 \mathrm{~F}$ ), ils s'engageaient à installer tout l'appareillage atmosphérique : machine fixe avec ses chaudières et la pompe à air, tube en fonte de quinze pouces de diamètre $(38 \mathrm{~cm})$, assemblé par longueurs de dix pieds (trois mètres) et un télégraphe électrique ${ }^{69}$. Cet appareillage devait être à même de tracter une charge totale de 26 tons $(26417 \mathrm{~kg})$ à une vitesse de 30 milles à l'heure $(47 \mathrm{~km} / \mathrm{h})$. Le prix comprenait une somme de $£ 2500$ comme dépôt de garantie, abandonnée à la compagnie en cas de «non-performance ». Au cas où la compagnie déciderait par la suite d'appliquer leur système sur la première partie du chemin entre Dublin et Kingstown, Clegg et les Samuda ne demanderaient pas de royalties pour cette nouvelle application de leur brevet. Ils proposaient en plus de reverser à la compagnie $10 \%$ de toutes les licences qui pourraient être accordées pour l'exploitation de leur brevet en Grande-Bretagne ou en Irlande. Pour la compagnie, cette offre constituait un appât considérable : le système était peut-être l'avenir des chemins de fer, le brevet une mine d'or potentielle.

Le projet reçut à ce moment-là l'encouragement non négligeable d'un rapport officiel sur les railways atmosphériques, commandé par le département des chemins de fer au Board of Trade et publié le 15 février 1842 par le lieutenant-colonel Sir Frederick Smith, ingénieur militaire, et Peter Barlow, professeur de mathématiques à l'Académie 
militaire de Woolwich. Ce rapport était favorable au système, qui paraissait avoir fait ses preuves techniques à Wormwood Scrubs. Certes, les coûts d'installation pouvaient être élevés, en raison des dépenses du tube et des machines fixes, mais l'investissement de départ serait sans doute compensé par les économies dans les frais d'exploitation, surtout pour une ligne avec des trains fréquents. Cependant, plusieurs questions pratiques n'étaient pas encore résolues (passages à niveau, embranchements, arrêts en gare...) et les avantages économiques du système dans son ensemble restaient à démontrer. Très opportunément, les deux auteurs concluaient que le principe paraissait particulièrement approprié au chemin de fer projeté entre Kingstown et Dalkey $^{70}$. Fort de cette recommandation, James Pim, de son côté, put négocier auprès du Board of Public Works un emprunt de $£ 25000$ (625000 F), remboursable sur 28 ans. Autre encouragement officiel enfin, venant des Commissioners du port royal de Kingstown: l'autorisation d'utiliser pour le chemin atmosphérique l'une des deux lignes de tramway existantes, que les Commissioners étaient prêts à vendre à la compagnie pour un prix très modéré. Sans représenter un investissement direct dans l'invention, contraire aux principes des pouvoirs publics britanniques en matière d'innovation technique, cette décision dispensait la compagnie de la coûteuse course d'obstacles qu'était l'obtention d'un nouvel acte du Parlement. En matière de trafic, enfin, la compagnie notait les nombreux projets de constructions résidentielles autour de Dalkey, conséquence « naturelle » de la venue du chemin de fer et laissant présager de nouveaux passagers, empruntant la ligne quotidiennement à des fins autres que celles de « divertissement et de plaisir ${ }^{71}$ ».

Construite par William Fairbairn, de Manchester ${ }^{72}$, dotée d'un volant énorme de 36 pieds de diamètre $(11 \mathrm{~m})$ et actionnant une pompe avec un cylindre de $66 \frac{1}{2}$ pouces $(1,67 \mathrm{~m})$, dans lequel la course du piston était de $66^{1 / 2}$ pouces, la machine verticale installée par les Samuda ${ }^{73}$ développait cent chevaux-vapeur, apparemment en excès des besoins du tube de propulsion qui ne mesurait que 2490 yards de long $(2275,86 \mathrm{~m})$. Mais cette machine, installée à proximité de l'arrivée de la ligne à Dalkey, était prévue pour servir à l'application du système sur la partie de la ligne entre Dublin à Kingstown et, aussi, pour une possible extension du chemin au-delà de Dalkey, contournant les collines de Killiney en direction de Bray, six milles $(9,3 \mathrm{~km})$ plus loin, dans de magnifiques paysages littoraux comparables à ceux de la baie de Naples ${ }^{74}$. La machine fixe fut mise en marche le 17 août 1843, le premier convoi atmosphérique aspiré le lendemain. Le samedi 19 août, d'immenses foules se rassemblaient le long du parcours pour voir un train de trois voitures monter à Dalkey, une centaine de passagers à bord et Joseph Samuda dans la voiture de tête. Le 21 août, un train atteignit la joyeuse vitesse de cinquante milles à l'heure $(80 \mathrm{~km} / \mathrm{h})$. Comme le remarquait le Journal des Chemins de Fer, rendant compte de ces essais prometteurs dans son numéro du 26 août 1843 , « on sait qu'une grande vitesse sur les chemins de fer produit l'hilarité chez les voyageurs. » 
Figure 22

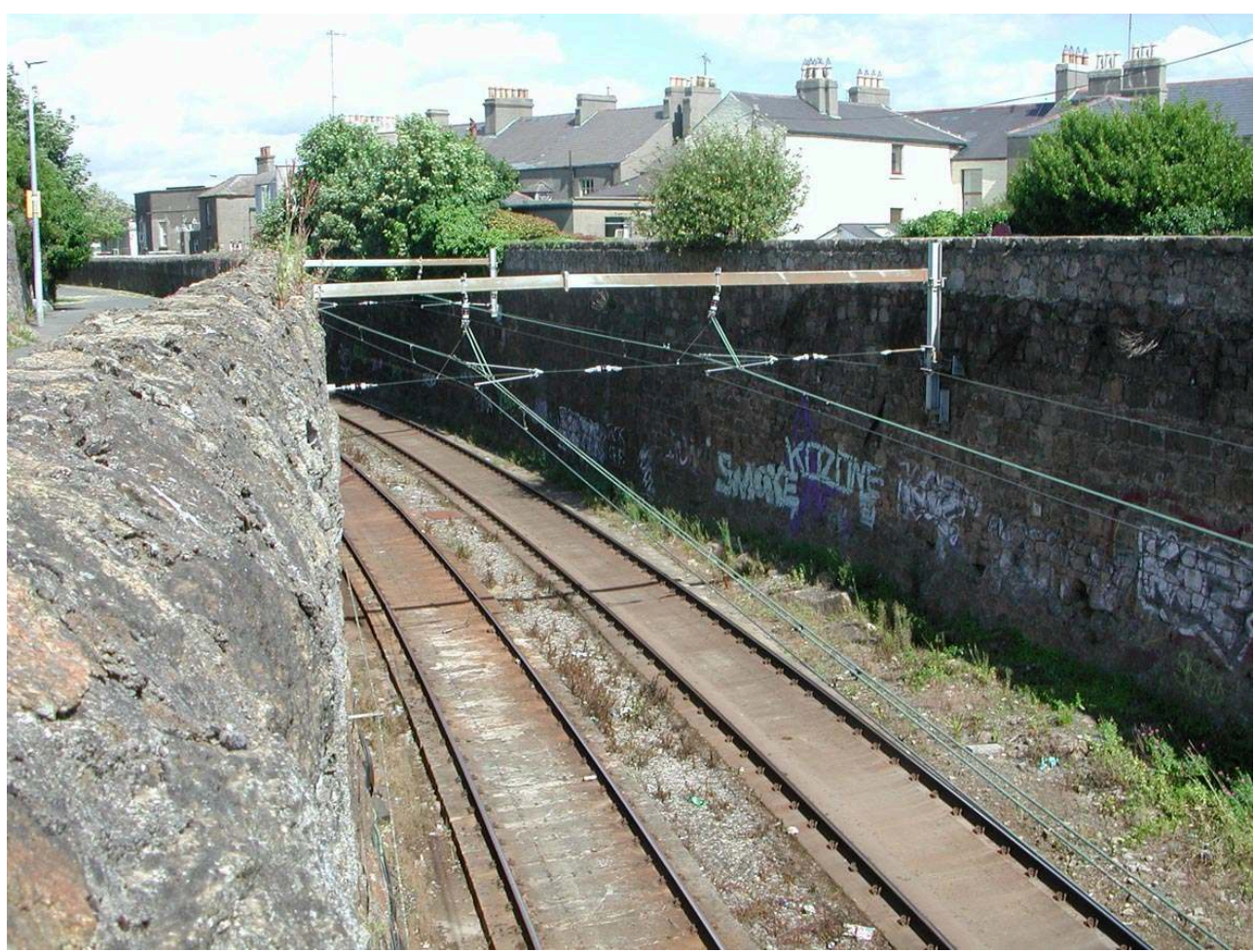

Tranchée de la ligne du chemin de fer atmosphérique près du port de Kingstown (Dún Laoghaire). Elle fut élargie en 1878 en vue du dédoublement de la voie. Photo Paul Smith, 2007.

(c) Paul Smith.

Les essais se poursuivaient pendant l'automne et l'hiver car, retardée par des travaux de couverture de la ligne, passant le long du port dans une tranchée de neuf pieds de profondeur (2,7 m) (fig. $\mathbf{n}^{\circ} \mathbf{2 2}$ ), l'ouverture officielle au public n'eut lieu que le 29 mars 1844. Des départs toutes les demi-heures de $8 \mathrm{~h}$ du matin à $6 \mathrm{~h}$ du soir et jusqu'à $9 \mathrm{~h}$ en été, furent annoncés, avec une interruption le dimanche entre midi et $2 \mathrm{~h}$ de l'aprèsmidi, au moment des offices religieux. Les tarifs furent fixés à deux pence en troisième classe et trois pence en deuxième; il n'y avait pas de première classe ${ }^{75}$. Ce retard, d'après James $\mathrm{Pim}^{76}$, avait été mis à profit pour mieux familiariser les servants de la compagnie à leurs tâches et pour conforter la confiance du public dans le nouveau mode de locomotion. La presse de Dublin fit d'emblée preuve d'une confiance presque sans bornes. The Dublin Evening Mail parla «du plus grand triomphe de la science moderne", rendant hommage à l'Irlande pour avoir pris les risques de l'essai, dorénavant un exemple pour le reste du monde. Le public s'habituait déjà, constata le journal, à un moyen de transport agréable, sûr, sans aucun balancement dans les voitures et sans aucun bruit susceptible d'interrompre la conversation. Le monde entier était redevable aux Samuda pour leur découverte admirable; l'esprit ne concevait aucune limite aux conséquences de cette invention pour la promotion des rapports humains et pour la prospérité de toutes les nations ${ }^{77}$. The Dublin Evening Post, quant à lui, pensait que l'adoption générale des chemins de fer atmosphériques n'était plus qu'une question de temps, annonçant la création imminente d'une ligne entre Dublin et Cork et reléguant déjà les locomotives dans la catégorie des "choses du passé ${ }^{78}$ ». Cet enthousiasme n'était pas qu'irlandais. Un article publié dans The Illustrated London News le 6 janvier 1844 allait dans le sens de la confiance attendue par Pim, parlant lui aussi 
d'un «triomphe de la science : la propulsion atmosphérique sur les chemins de fer est dorénavant un fait accompli... En faisant la part des promesses exagérées des promoteurs du système, et en nous appuyant sur ce qui a été réellement accompli, ce système est capable d'être appliqué et offre un moyen de transport moins cher, plus sûr et plus commode que les chemins de fer à locomotives. » (fig. $\mathbf{n}^{\circ} \mathbf{2 3}$ ).

Figure 23

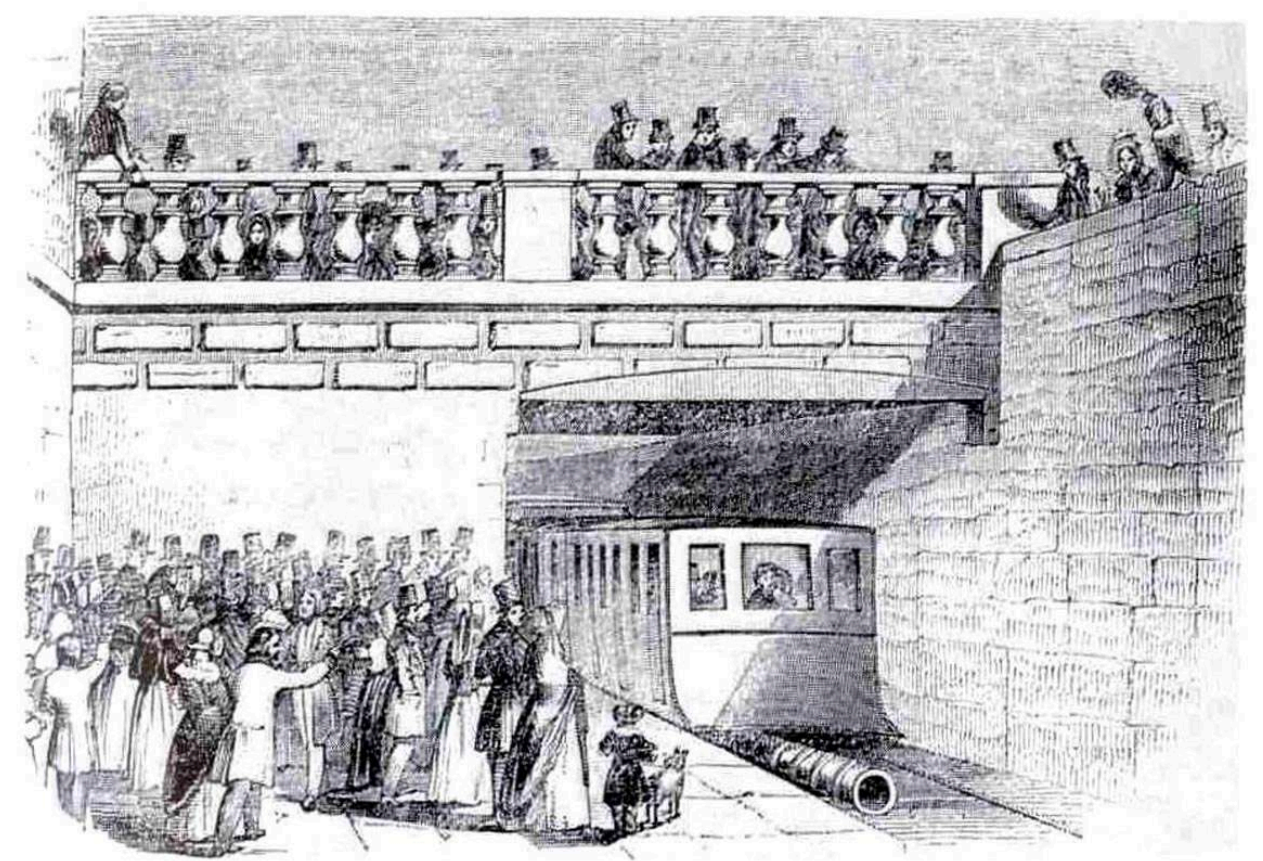

Le départ du train atmosphérique de la gare de Kingstown, gravure d'après une esquisse de James Mahony, THE ILLUSTRATEd LONDON NEWS, 6 janvier 1844. Repro Paul Smith, 2008.

(C) Paul Smith.

Il y eut cependant quelques difficultés de démarrage. Le tube de propulsion s'arrêtait à 560 yards $(512 \mathrm{~m})$ du terminus à Dalkey, la vitesse acquise par le convoi étant censée l'amener en gare. Pour le retour, les trains étaient abandonnés à la gravité, avec le piston rétracté. Mais parfois, à Dalkey, les passagers durent être réquisitionnés pour pousser le train jusqu'au quai. Parfois, au contraire, freinant avec difficulté, le train dépassait la gare et quittait même les rails. Chaque voiture était équipée de freins mais, pour les faire fonctionner, les garde-freins devaient obéir au son d'un coup de sifflet donné par le conducteur principal. Il y eut également des accidents : « un garde-freins a eu la jambe serrée entre deux voitures et a été sérieusement blessé ${ }^{79}$." À une autre occasion, par mégarde, le wagon conducteur partit de Kingstown sans être attaché à ses wagons de passagers et fut aspiré à la vitesse vertigineuse de 84 milles à l'heure $(135 \mathrm{~km} / \mathrm{h})$, sans doute un record mondial de vitesse pour l'époque ${ }^{80}$.

41 Cette mésaventure illustrait l'un des handicaps intrinsèques du système, à savoir l'absence de maitrise, par le conducteur, de la force motrice propulsant son train. Le chauffeur et le mécanicien de la machine fixe mettaient celle-ci en marche en fonction des horaires annoncés, sans connaître l'état de préparation du convoi à Kingstown. Posté à la fenêtre du bâtiment des machines à Dalkey, un jeune garçon annonçait l'approche du train pour qu'ils fissent cesser l'action de la pompe (fig. $\mathbf{n}^{\circ} \mathbf{2 4}$ ). 
Figure 24

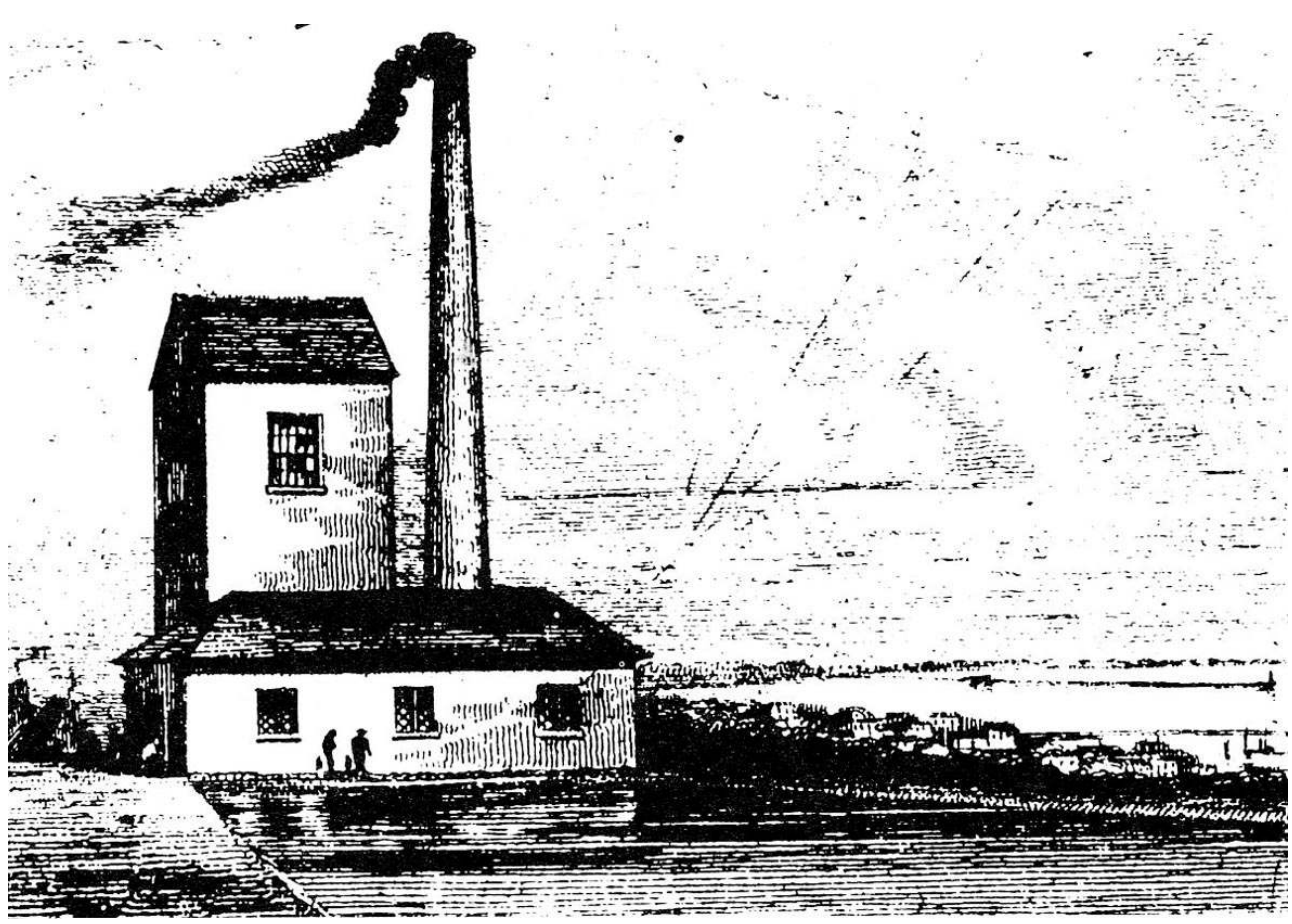

Le bâtiment des machines à Dalkey, avec son bassin d'eau au premier plan. D'après une gravure par Kirkwood, reproduite par K. A. Murray, IRELAND's FIRST RAILWAY, 1981, p. 57. Repro Paul Smith, 2008. (c) Paul Smith.

Apparemment, le télégraphe électrique promis n'était pas encore installé lorsque Jacob Samuda décrivit le fonctionnement de la ligne à l'Institution of Civil Engineers en mai 1844. En revanche, l'autre faiblesse majeure du système, l'impossible étanchéité de la soupape, ne paraissait pas rédhibitoire. Selon Samuda, les infiltrations d'air dans le tube n'absorbaient qu'une force égale à cinq chevaux-vapeur par mille, aisément compensée par leur machine de cent chevaux. Deux agents - "valvemen », des gardessoupape - étaient employés en permanence pour l'entretien de la soupape longitudinale et le renouvellement de la composition d'étanchéité.

Un autre avantage inattendu du retard imposé à l'ouverture de la ligne résidait dans l'opportunité qu'il avait offerte pour des observations et des expériences par des " professional gentlemen ». De nombreuses compagnies ferroviaires anglaises, ainsi que « la plupart des gouvernements d'Europe " avaient envoyé ingénieurs et enquêteurs à Kingstown. Comme les artistes allaient à Rome, pour les spécialistes-ès-chemins de fer, le petit village irlandais de Dalkey devint, en 1843 et 1844, un lieu de pèlerinage obligé. Mais, si la compagnie elle-même rendait hommage à Clegg et Samuda pour la manière hautement satisfaisante avec laquelle ils s'étaient acquittés de leurs engagements, et se montrait par la suite tout à fait contente du fonctionnement du système ${ }^{81}$ et des bénéfices qu'il procurait ${ }^{82}$, tous les visiteurs n'étaient pas convaincus.

\section{Différences atmosphériques}

En effet, les essais en Irlande divisaient les spécialistes en deux camps, dont les arguments contradictoires se déployaient dans la presse spécialisée, dans des 
brochures, dans plusieurs débats organisés à l'Institution of Civil Engineers à Londres en 1844 et 1845, et devant une commission parlementaire siégeant en mai 1844 à l'occasion d'un projet de loi pour une nouvelle ligne atmosphérique au sud de Londres, vers Epsom. Parmi les sceptiques, le "père » des chemins de fer, George Stephenson, pour qui l'atmosphérique n'était que "fumisterie [humbug], du début à la fin ${ }^{83}$ ». L'« élève » de Stephenson, Joseph Locke ${ }^{84}$, ingénieur en chef de la ligne de Paris à Rouen inaugurée en mai 1843 et prolongée ensuite jusqu'au Havre, figurait également parmi les sceptiques, tout comme le principal entrepreneur de construction de cette ligne, l'Anglais William Mackenzie. Ce dernier put visiter le chemin de fer de Kingstown à Dalkey le 13 mai 1845 : son verdict : « the most Zig Zag narrow disgraceful thing I ever beheld ${ }^{85}$.» Cette hostilité dédaigneuse se comprend: la réussite du système atmosphérique menacerait directement le métier de "contractor», c'est-à-dire d'entrepreneur, dans laquelle Mackenzie faisait alors fortune grâce au système des locomotives, avec ses immenses besoins en matière de terrassements et d'ouvrages d'art.

Figure 25

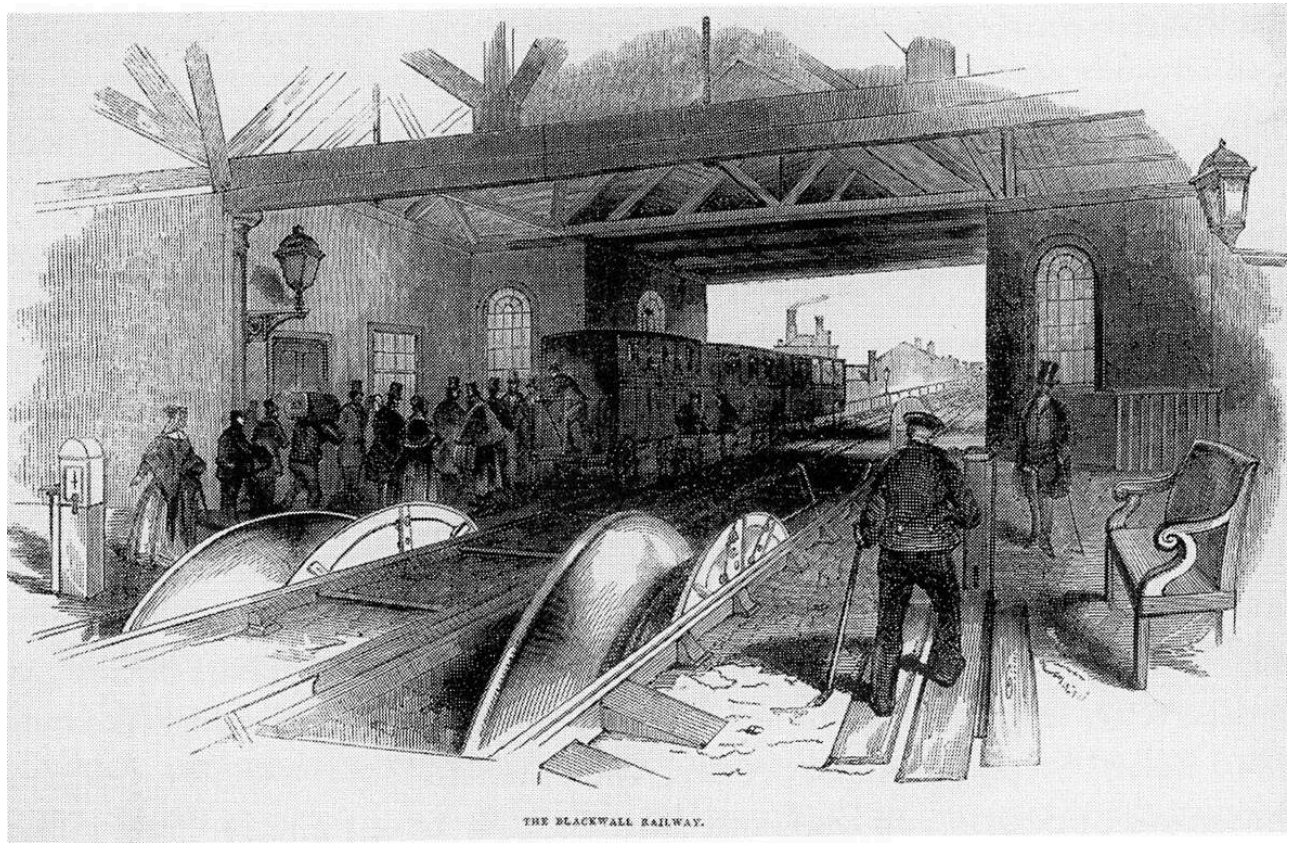

Ouvert en 1840, le London \& Blackwall Railway de Robert Stephenson. Les immenses tambours pour l'enroulement de cordes de traction sont visibles au premier plan. Gravure reproduite dans BAILEY, Michael R. ROBert STePhenson, THe EMINent engineer, 2003, p. 194. Repro Paul Smith, 2008. (C) Paul Smith.

Robert Stephenson, le fils de George, devint également un adversaire du système atmosphérique, mais pas avant d'avoir observé de près la ligne irlandaise. Présenté souvent comme une alternative au système employant des machines fixes pour tracter des trains par corde ou par câble - système dont Stephenson était lui-même l'un des partisans, ayant installé deux lignes de ce type à Londres ${ }^{86}\left(\right.$ fig. $\left.\mathbf{n}^{\circ} \mathbf{2 5}\right)$ - le système atmosphérique, aux yeux de cet éminent ingénieur, pouvait être considéré, suivant l'expression utilisée par Charles Vignoles dès 1842, comme une "corde d'air » dont la concurrence potentielle méritait toute son attention. En novembre 1843, pour le compte des directeurs de la compagnie du Chester \& Holyhead Railway, il visita le 
chemin atmosphérique de Kingstown à Dalkey, accueilli par l'état-major de la compagnie comprenant Pim, Bergin, Clegg, Samuda et Bonfil, et par un grand nombre de personnes intéressées par les expériences qu'il allait effectuer ${ }^{87}$. En février et mars 1844, pour effectuer des essais et des mesures plus détaillées, Stephenson envoya en Irlande deux de ses collaborateurs, George Berkley et William Marshall, le premier ayant travaillé auparavant sous les ordres de Jacob Samuda à Southwark ${ }^{88}$. Les conclusions tirées de toutes ces investigations furent consignées dans un rapport publié par Stephenson en avril 1844, envisageant l'ensemble de la question d'un point de vue commercial autant que scientifique ${ }^{89}$. Tout en qualifiant l'invention de Clegg et Samuda de "singulièrement ingénieuse et hautement méritoire " et reconnaissant l'incontestable supériorité du système atmosphérique en matière de sécurité, Stephenson déconseillait son usage sur la ligne envisagée pour le Chester \& Holyhead. S'appuyant, pour étayer ses comparaisons de coûts, sur le chemin de fer de Londres à Birmingham dont il avait été ingénieur en chef de 1834 à 1838, il conclut que, contrairement à ce que prétendaient les défenseurs du système atmosphérique, les dépenses d'installation et d'exploitation de celui-ci seraient nettement supérieures à celles du système des locomotives. Supposant un chemin d'une centaine de milles, une machine fixe tous les trois milles et demi et des trains partant chaque demi-heure pour avancer à une vitesse moyenne de 37 milles à l'heure $(60 \mathrm{~km} / \mathrm{h})$, chacun de ces trains en croiserait onze venant en sens inverse. Dès lors, sur une telle grande ligne, la voie unique prônée pour les chemins de fer atmosphériques était inconcevable ou, du moins, très malavisée. D'emblée, les estimations de Clegg et Samuda quant aux frais d'installation d'un chemin atmosphérique étaient à multiplier par deux. En outre, contrairement à ce qu'avançait Jacob Samuda, Stephenson calcula que les fuites d'air dans le tube et dans la pompe représentaient, sur la ligne irlandaise, une perte de puissance allant jusqu'à $74 \%$, et qui serait plus grande encore sur une ligne plus longue. Ces pertes de puissance étaient plus importantes que celles constatées sur son propre chemin à traction par câble entre Camden et la gare d'Euston, estimées à $30 \%$. Même pour une petite ligne à câble comme celle de Blackwall, le système atmosphérique serait peu pratique, étant donné les arrêts fréquents à des stations intermédiaires. Tout au plus reconnaissait-il que pour une ligne courte dans le voisinage d'une grande ville, nécessitant des services rapides et fréquents entre deux gares terminus (une navette, dirait-on aujourd'hui), ou encore sur d'autres lignes courtes où des pentes accusées interdisaient encore l'usage des locomotives, le système atmosphérique pourrait être éligible. Ainsi approuvait-il une proposition faite par Brunel pour utiliser le système atmosphérique sur une ligne entre Gênes et Turin, où, en traversant les Apennins, il y aurait une rampe continue de $1,6 \mathrm{~cm}$ par mètre ${ }^{90}$. Mais, pour une grande ligne de 85 milles ( $137 \mathrm{~km})$, comme celle projetée par les directeurs de la compagnie auxquels Stephenson rendait son rapport, le système atmosphérique était à exclure, surtout du fait de son manque de flexibilité. Le bon fonctionnement de l'ensemble dépendrait de la performance parfaite de chaque élément de l'appareillage : la moindre panne arrivée à une machine fixe, à une pompe, au piston ou sur un point donné de la soupape longitudinale bloquerait toute circulation.

Stephenson, pourrait-on objecter - et l'objection lui fut faite lors d'une enquête parlementaire de mai 1844 , et par d'autres auteurs ensuite ${ }^{91}$-, n'était pas un témoin désintéressé : ses ateliers de construction de locomotives, fondés à Newcastle-uponTyne en 1824 et devenus en 1840 les plus importants de l'Angleterre, ne le disposaient guère à voir avec bienveillance un système qui annonçait la mort des locomotives. Mais, 
étant donné sa grande réputation, son rapport, diffusé dans la Mechanics' Magazine du 15 juin 1844, eut un impact considérable, que Jacob Samuda cherchait à contrer en contestant ses données expérimentales, et que Samuel Clegg réfutait en citant la satisfaction exprimée par les directeurs de la ligne irlandaise et les avis «impartiaux » d'autres témoins réputés ${ }^{92}$.

Parmi les ingénieurs favorables au système atmosphérique, on comptait, bien évidemment, le professeur Charles Vignoles, parti à Würtemberg peu après l'achèvement des travaux en Irlande pour soutenir un projet de ligne atmosphérique à Stuttgart. Le major-general Pasley, inspecteur-général des chemins de fer, annonça au Board of Trade qu'il trouvait le système parfaitement efficace et sûr ${ }^{93}$. James Walker, président de l'Institution of Civil Engineers, dans son adresse à l'assemblée générale annuelle du 16 janvier 1844, rendait compte de sa récente visite à Kingstown ${ }^{94}$ et, sans vouloir faire des pronostics généraux - autre que l'intérêt que devait sûrement susciter la question auprès des philosophes et des ingénieurs curieux des lois de la nature et de leur application aux besoins de l'homme -, notait quand même que les résultats des essais atmosphériques paraissaient bien mieux avancés que ceux des locomotives à vapeur à une époque comparable de l'histoire de leur introduction ${ }^{95}$. Même le Premier ministre, Sir Robert Peel, lors d'un débat sur les chemins de fer à la Chambre des communes, le 5 février 1844, se déclara favorable à l'invention - « due à deux frères de confession juive" -, constatant le succès de son application en Irlande et conseillant aux compagnies ferroviaires d'être attentives à ce système de pression atmosphérique ${ }^{96}$.

\section{William Cubitt et le London \& Croydon}

Deux autres ingénieurs aux réputations confirmées s'étaient déclarés partisans des chemins de fer atmosphériques, persuadés des avantages de l'application du système sur des lignes ordinaires avec des trains circulant dans les deux sens. Le premier était William Cubitt, ingénieur civil né en 1785, diversement connu pour ses travaux sur les ailes des moulins à vent, pour l'installation d'usines à gaz et pour son invention du « treadmill », le moulin à discipline employé dans des prisons anglaises à partir de 1819. Passé des canaux aux chemins de fer, il fut l'ingénieur du South Eastern Railway entre Londres et Douvres, et, en 1847, avec Bazaine, ingénieur consultant pour la ligne française d'Amiens à Boulogne, concédée à la compagnie de Charles Laffitte et Edouard Blount. Sa conversion atmosphérique datait des essais à Wormwood Scrubs et, en novembre 1841, son avis d'expert fut mis en exergue par James Pim dans sa campagne en faveur de l'introduction du système entre Kingstown et Dalkey: "Je ne vois aucune objection fondamentale à la théorie du Railway Atmosphérique, écrivit Cubitt, et, à mon avis, les difficultés mécaniques sont susceptibles d'être surmontées par les moyens proposés par les détenteurs du brevet ${ }^{97}$. "

49 Par la suite, conforté dans ces vues par l'apparente réussite du chemin atmosphérique irlandais, Cubitt parvint à convaincre le président de la London \& Croydon Railway Company, William A. Wilkinson, de tenter le procédé sur une ligne d'une vingtaine de milles au sud de Londres, partant de Forest Hill pour aboutir à Epsom, localité connue pour son grand rendez-vous hippique et mondain, le Derby, couru tous les ans vers la fin du mois de mai ${ }^{98}$. Suivant le rapport de la commission parlementaire de mai 1844 , cette ligne fut autorisée par un acte du Parlement du 6 juin. Secondé par son fils Joseph, 
William Cubitt fut nommé ingénieur de la ligne qui devait donc utiliser le système breveté par Clegg et Samuda. Avec Joseph Clegg lui-même comme ingénieur conseil, cinq milles de ligne furent d'abord réalisés, jusqu'à Croydon, employant, comme à Dalkey, un tube de quinze pouces de diamètre $(38 \mathrm{~cm})$, fourni par les célèbres fonderies de Coalbrookdale. Il s'agissait, cette fois-ci, d'une ligne de banlieue, anticipant des services fréquents pour desservir trois stations intermédiaires mais employant une voie unique, pour laquelle quatre stations de pompage d'air furent édifiées, dont trois seulement mises en activité. Pour passer par-dessus la ligne existante vers Brighton et Douvres, ce chemin de Croydon comportait à Norwood un saut-de-mouton construit en bois, l'une des premières structures de ce genre dans l'histoire des chemins de fer, permettant d'éviter des aiguillages compliqués ${ }^{99}$ (fig. $\mathbf{n}^{\circ} \mathbf{2 6}$ ).

Figure 26

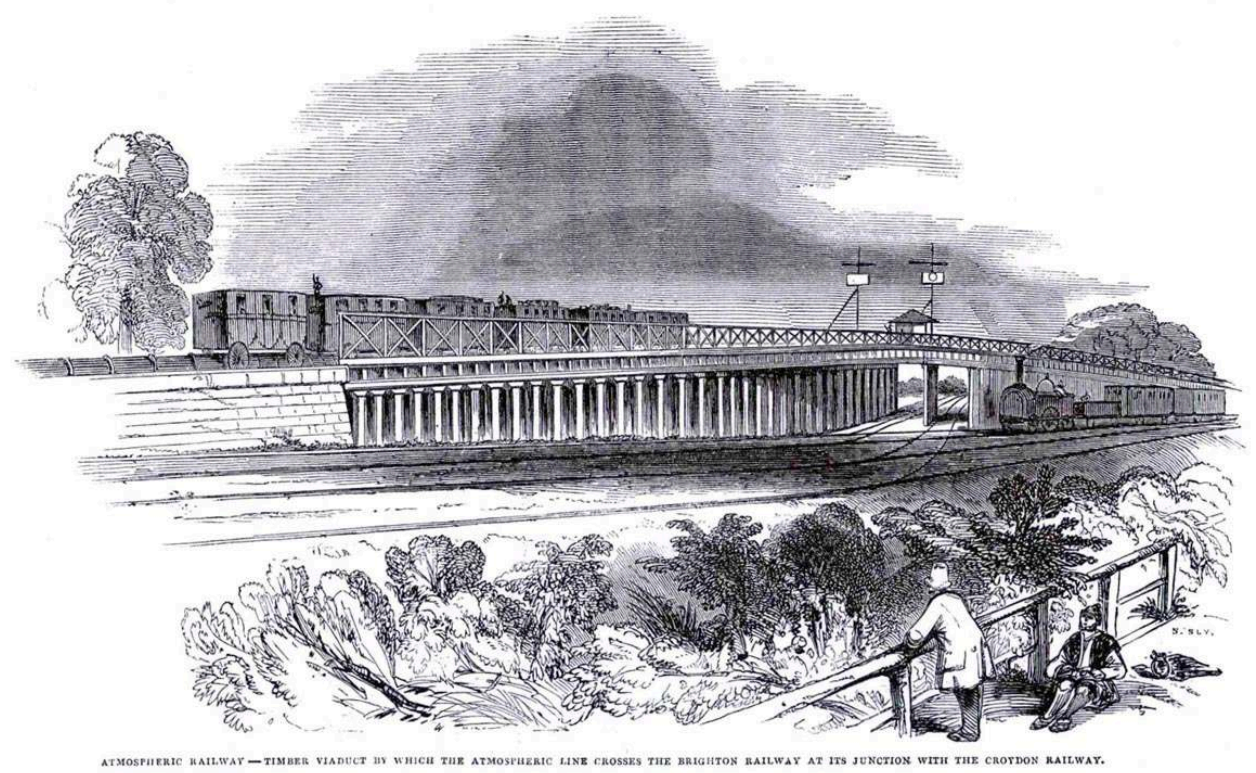

Le saut de mouton à Norwood Junction, un train atmosphérique passant par-dessus la ligne de Brighton et Douvres. Dossier sur le chemin de fer atmosphérique à Croydon Public Library.

(C) Croydon Public Library.

Les premiers essais eurent lieu en août 1845, notamment pour tester le croisement des convois dans les stations intermédiaires, où le tube devait être interrompu. Les voies d'évitement nécessitaient la conception par Cubitt d'aiguillages dont on imagine la complexité : six hommes étaient nécessaires pour en manœuvrer les leviers ${ }^{100}$. La ligne fut ouverte au public en janvier 1846, avec trente-deux trains par jour en mars. Mais, dès le mois de mai, le fonctionnement de la ligne montrait d'inquiétants signes de faiblesse : les plaques de fer protectrices de la soupape longitudinale résistaient mal à l'usure quotidienne, et, en se cassant, déchiraient le cuir de la soupape ; surmenées, les machines fixes connaissaient de nombreuses avaries ${ }^{101}$. Au cours de l'été, de fortes chaleurs rendaient inopérante la composition à base de cire d'abeilles et de suif. Pendant un arrêt des services atmosphériques de six semaines, Samuda fut contraint de remplacer le cuir de la soupape et d'étudier une nouvelle composition d'étanchéité. Enfin, au début du mois de mai 1847, la compagnie de London \& Croydon, dorénavant amalgamée dans une nouvelle compagnie, la London, Brighton and South Coast, 
annonça l'abandon définitif du système et son remplacement par un service à locomotives; elle redouta notamment les défaillances de l'appareillage atmosphérique lors de la journée des courses à Epsom à la fin du mois.

Pour de nombreux observateurs, cet arrêt soudain marqua déjà la fin définitive de l'aventure atmosphérique ${ }^{102}$. Le Mechanics' Magazine, revenu de son enthousiasme initial pour le système, publia une amusante notice de nécrologie scientifique, annonçant le décès, à Croydon, de Mademoiselle Vacuana Airy, la célèbre "Fainting Lady », la dame qui s'évanouissait. Sa santé n'avait jamais été très bonne car elle souffrait d'une espèce d'asthme constitutionnel qui la faisait respirer avec beaucoup de difficulté, ses lèvres toujours ouvertes. Dernièrement, son état avait présenté un état pitoyable de faiblesse mais ses lèvres, hélas, étaient dorénavant scellées pour toujours. Les obsèques étaient annoncées, justement, pour le prochain Derby Day. Le deuil devait être conduit par Jacob Samuda et James Pim et les cordons du poêle seraient tenus par William Cubitt, Isambard Kingdom Brunel et William A. Wilkinson ${ }^{103}$. Herepath's Railway Journal, hostile dès le départ à tout ce qui était atmosphérique, proposa de reconvertir en synagogue l'un des bâtiments de pompage: Joseph Samuda pourrait y prier pour se faire pardonner le gaspillage d'autant d'argent des actionnaires ${ }^{104}$.

Il convient ici, en passant, de noter l'habillage architectural donné à ces bâtiments de pompage. En effet, afin de rassurer « les amoureux de la douce rusticité des paysages anglais, dont le caractère pittoresque serait détruit par un aspect à cheminée [chimneyed aspect], si singulièrement indicatif des localités manufacturières ${ }^{105} 》$, les machines fixes de cette ligne de banlieue furent abritées dans des édifices dessinés par W. H. Breakspear, architecte de la compagnie, et Raphael Brandon, responsable des détails architecturaux des bâtiments. Ceux-ci présentaient l'apparence de petites églises néo-gothiques, la cheminée ayant la forme d'une flèche ornée de crénelures et d'un clocheton. De manière révélatrice, les gravures de l'époque qui représentent ces cheminées gothiques ne montrent pas l'épaisse fumée qui en sortait. Les stations de la ligne, quant à elles, étaient conçues dans le style des manoirs médiévaux (fig. $\mathbf{n}^{\circ} \mathbf{2 7}, \mathbf{2 8}$, 29). 
Figure 27

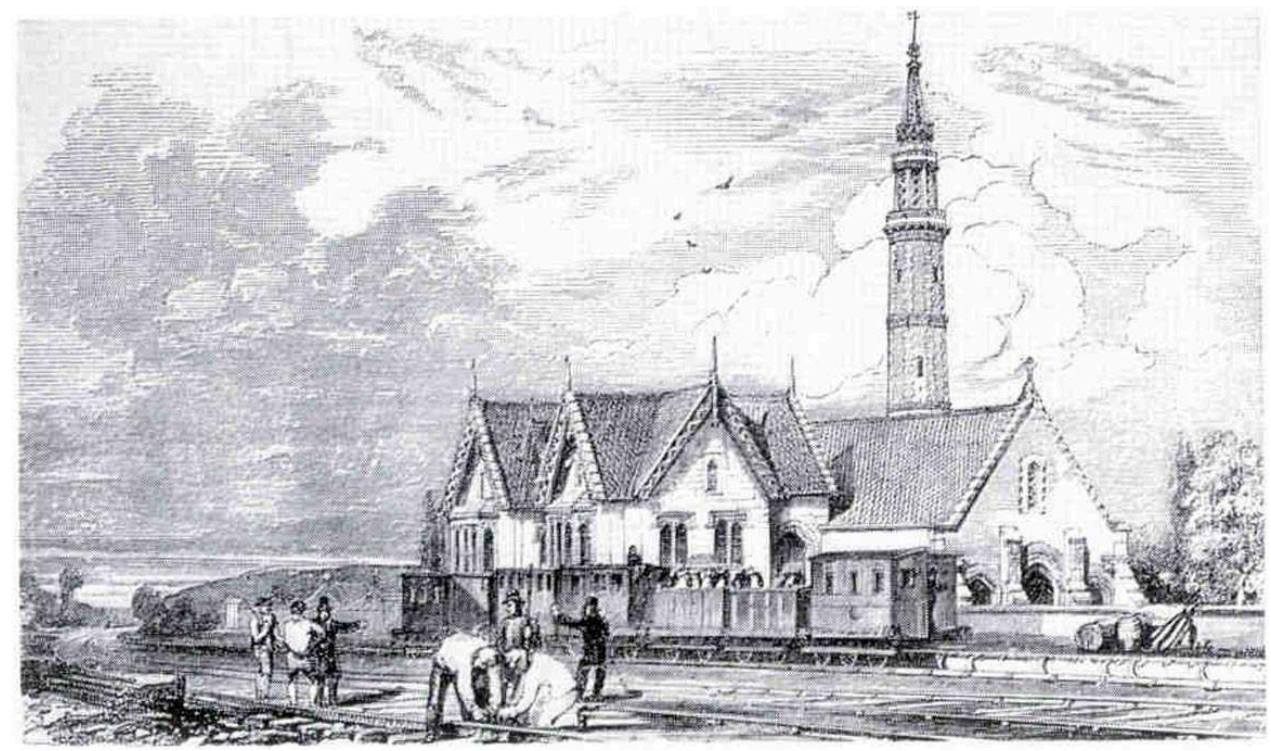

La gare de Forest Hill sur la ligne de Croydon, avec le bâtiment des machines et sa cheminée derrière. Dossier sur le chemin de fer atmosphérique à Croydon Public Library.

(c) Croydon Public Library.

\section{Figure 28}

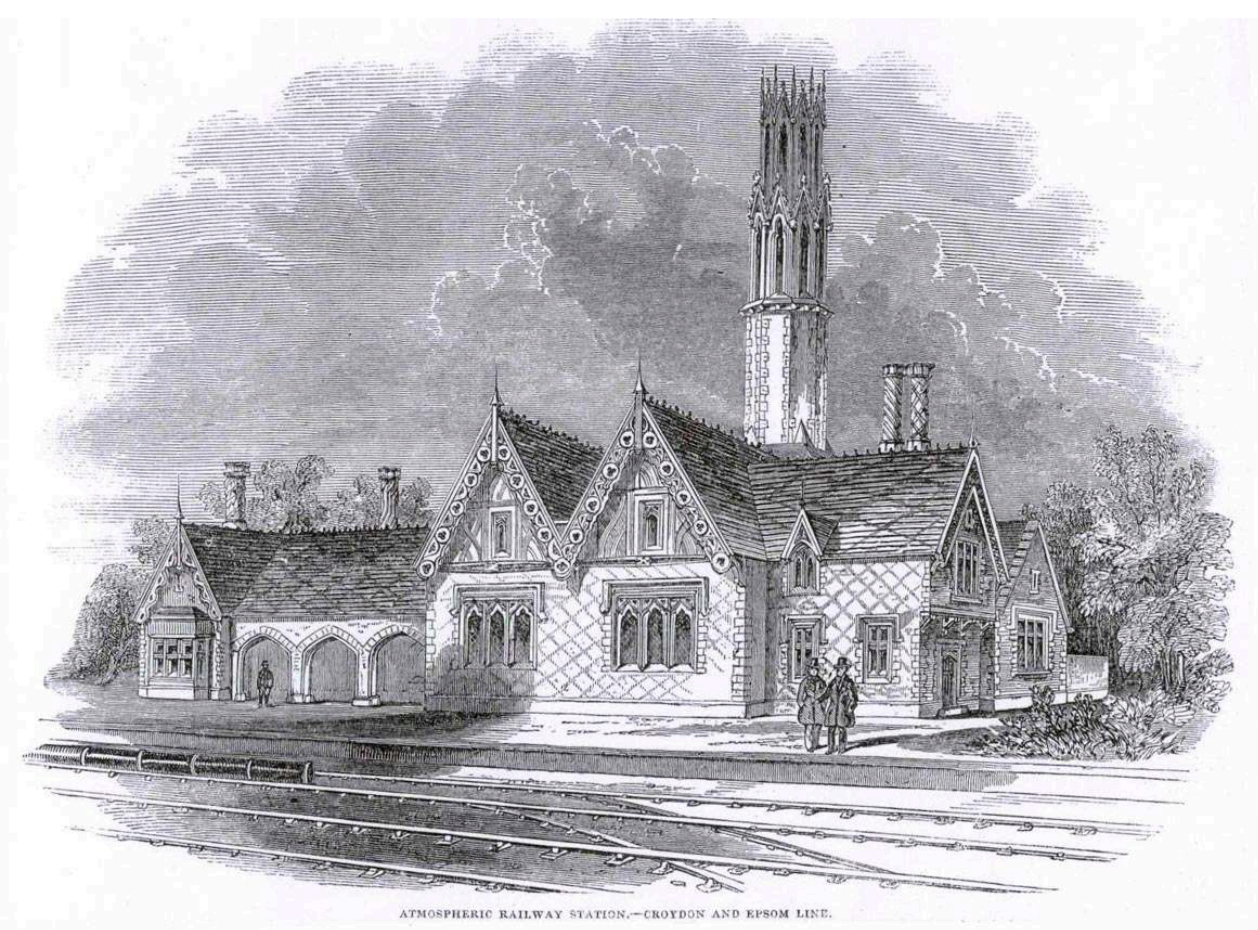

Une des gares sur la ligne de Croydon. Noter l'interruption du tube de propulsion en gare. Dossier sur le chemin de fer atmosphérique à Croydon Public Library.

(c) Croydon Public Library. 
Figure 29

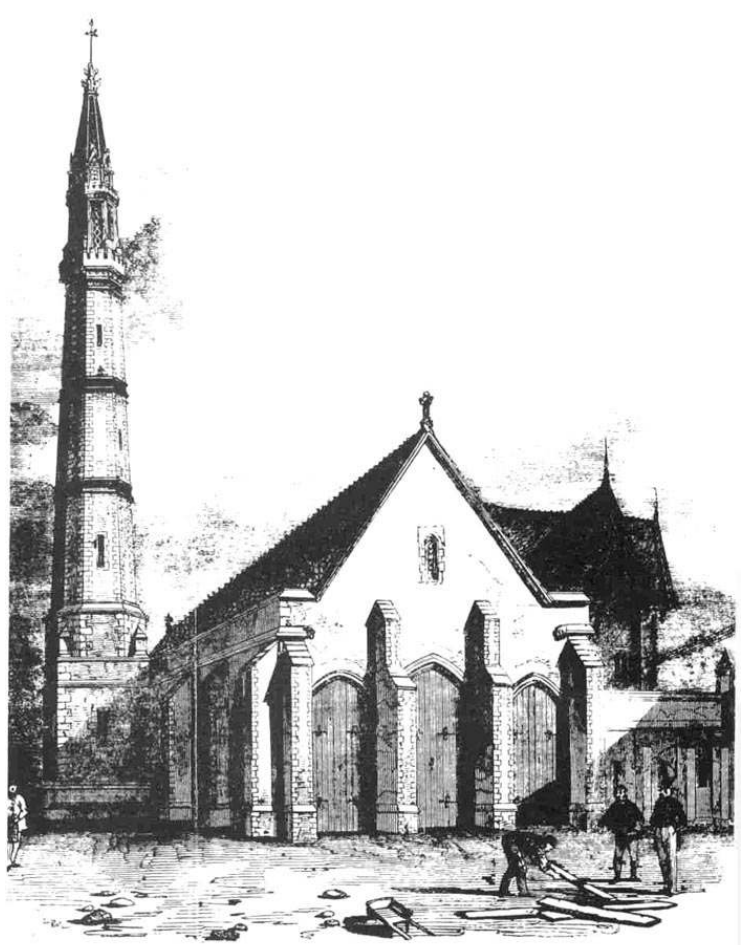

Le bâtiment des machines à Forest Hill sur la ligne de Croydon. Dossier sur le chemin de fer atmosphérique à Croydon Public Library.

(c) Croydon Public Library.

\section{Isambard Kingdom Brunel et le South Devon}




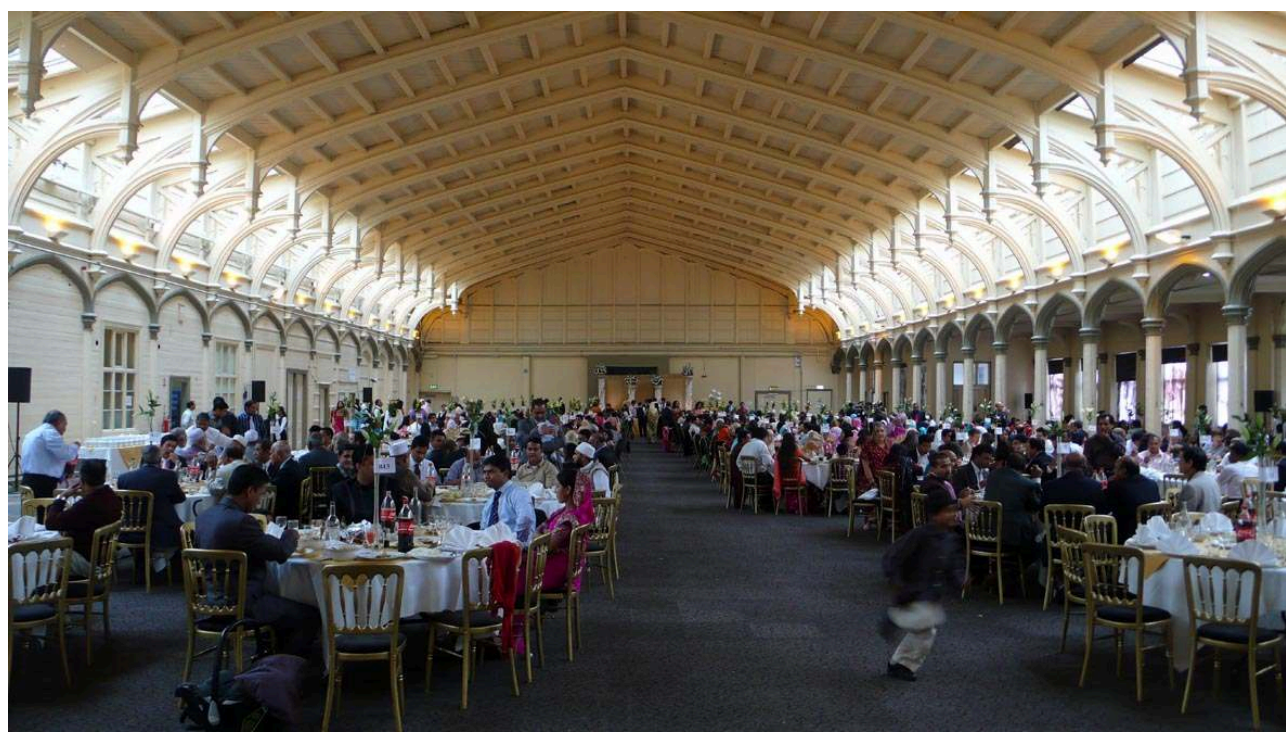

La gare de Temple Meads à Bristol, dessinée par Isambard Kingdom Brunel et ouverte en 1841. La halle aux trains avec sa charpente en bois est utilisée de nos jours pour des réceptions. Photo Paul Smith, 2008.

(c) Paul Smith.

Le second ingénieur converti à la cause atmosphérique était Isambard Kingdom Brunel. Malgré son jeune âge - il était né en 1806 -, Brunel était déjà célèbre pour le Great Western Railway, chemin de fer qui se distinguait par sa voie à grand écartement (7 pieds ou 2,133 m), préférée à l'écartement «standard » (4 pieds, 8 1/2 pouces ou 1,43 m) pour sa supériorité supposée en termes de vitesse et de confort. La ligne, ouverte sur toute sa longueur en 1841, s'étendait sur 118 milles (190 km) entre Londres-Paddington et Bristol-Temple Meads ${ }^{106}$ (fig. $\mathbf{n}^{\circ} \mathbf{3 0}$ ).

Brunel en avait été nommé ingénieur en 1833, sans aucune expérience préalable dans les chemins de fer mais déjà réputé pour sa participation aux travaux du tunnel sous la Tamise, conçu par son père, pour son projet de pont suspendu à Clifton, à Bristol, et pour son premier navire transatlantique, le Great Western. Sur les premiers tronçons du chemin de fer, en 1838, la ligne était exploitée avec des locomotives construites selon des spécifications dictées par Brunel lui-même. Ces locomotives s'avéraient exceptionnellement inefficaces, «la plus grande et plus inexplicable maladresse de sa carrière d'ingénieur" selon son biographe L.T.C. Rolt ${ }^{107}$. Laissant ensuite à Daniel Gooch, recruté en 1837, toute responsabilité pour les locomotives de la ligne, Brunel se montrait très attentif à l'émergence d'une technologie alternative de traction. Il connaissait les travaux de Pinkus et, à titre personnel, avait conduit quelques expériences sur le chemin d'essai à Wormwood Scrubs. En 1842, il préconisa l'usage du système atmosphérique sur certaines sections d'une ligne italienne. « M. Brunel, de la compagnie du Great Western, révéla le Journal des Chemins de Fer du 18 juin 1842, de retour de son voyage en Italie où il est allé étudier la voie de fer qu'on se propose d'établir entre Gênes, Milan et Turin, a l'intention d'appliquer le système atmosphérique à la partie de la ligne qui gravira les Apennins. Les chutes d'eau qu'on trouve en abondance dans ces montagnes seraient utilisées pour mettre en mouvement les pompes à air ${ }^{108}$ ». En novembre 1843, Brunel fit son premier pèlerinage à Dalkey, $y$ retournant en septembre 1844, accompagné de Daniel Gooch, qui se rangea aussitôt 
parmi les sceptiques ${ }^{109}$. En mai 1844, Brunel fut auditionné par la commission parlementaire à propos de la ligne de Croydon et Epsom, prenant la parole juste avant son ami Robert Stephenson, dont il venait de recevoir le rapport. Malgré les conclusions si négatives de celui-ci, Brunel persistait dans ses convictions quant à la supériorité du système atmosphérique en matière de vitesse, de sécurité et de confort. Il restait convaincu aussi qu'avec des machines fixes, et en l'absence de locomotives dépensant de l'énergie pour tracter leur propre poids mort, les coûts d'exploitation seraient moindres. Quant aux fuites dans la soupape longitudinale, elles étaient, selon ses propres observations à Dalkey, «si peu considérables qu'on pouvait sans danger ne pas s'en occuper ».

Le 4 juillet 1844, un acte du Parlement autorisa l'incorporation d'une nouvelle compagnie ferroviaire, le South Devon Railway, dont les directeurs, après l'envoi d'une députation à Dalkey, décidèrent d'appliquer le système atmosphérique sur toute la longueur de la ligne qu'ils projetaient, un parcours de 52 milles $(84 \mathrm{~km})$ entre Exeter et Plymouth. Longeant la rive droite de l'Exe et desservant les stations balnéaires de Dawlish et Teignmouth avant de passer par Newton ${ }^{110}$ et Totnes, cette ligne prolongerait une ligne existante qui bifurquait du Great Western à Bath. Une offre des frères Samuda pour la fourniture de la soupape longitudinale fut acceptée et Brunel nommé ingénieur. Dans un rapport adressé à la compagnie le 19 août $1844^{111}$, celui-ci prenait sur lui la pleine et entière responsabilité de la recommandation d'adopter le système atmosphérique, réitérant l'expression de sa confiance que les «simples difficultés mécaniques » dues à l'enfance du système pouvaient être surmontées. Dans des propos révélateurs de sa conception plutôt aristocratique des chemins de fer, il insista aussi sur les atouts du système en matière de confort: " Il me semble que la qualité du voyage sera grandement améliorée et que nous atteindrons des vitesses plus élevées avec moins de bruit et de secousses, et une absence de poussière de charbon, qui reste encore une nuisance considérable. Un encouragement sera ainsi créé pour ceux - la majorité des voyageurs - qui se déplacent soit pour de simples raisons de plaisir ou, du moins, pas par nécessité, et qui sont surtout influencés par le degré de confort dans lequel ils peuvent aller d'un endroit à l'autre.» Lors de la première assemblée générale des actionnaires de la compagnie, tenue au Royal Hotel à Plymouth le 28 août 1844, le président, Thomas Gill, annonça que les directeurs étaient persuadés qu'il y avait peu de chemins de fer en exploitation ou en projet qui offrissent de plus belles perspectives de réussite que leur ligne du South Devon ${ }^{112}$. 
Figure 31

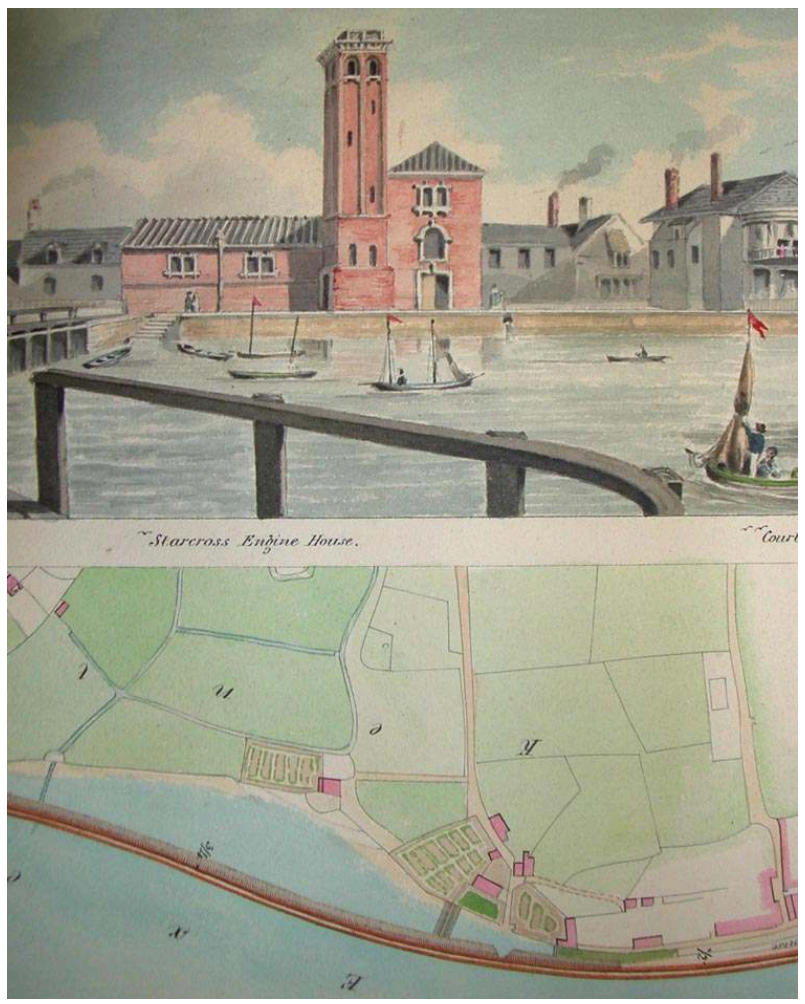

Le bâtiment des machines à Starcross. Détail d'une aquarelle de William Dawson, réalisée en 1847. Album conservé par l'Institution of Civil Engineers, London.

(C) Institution of Civil Engineers.

Épaulé par Joseph Samuda et ses collaborateurs venus de Croydon pour s'installer à Dawlish, à mi-parcours de la ligne, Brunel se lança dans les travaux avec la volonté manifeste d'attacher son propre nom au triomphe définitif du nouveau système de locomotion ferroviaire. Les machines fixes furent commandées à des firmes dont la réputation devait garantir la meilleure construction et qualité d'exécution: G. et J. Rennie, Maudslay Son \& Field, et Boulton \& Watt ${ }^{113}$. Pour abriter ces machines, avec leurs pompes et leurs chaudières, il dessina une série de bâtiments dans un style italianisant (fig. $\mathbf{n}^{\circ} \mathbf{3 1}$ ). Entourés d'un escalier en spirale, les fûts des cheminées, de section ronde, furent montés sans échafaudages à l'intérieur de tours carrées en forme de campaniles. Souvenir peut-être de son récent voyage transalpin, pendant lequel il avait pu visiter Rome et Venise ${ }^{114}$, ce style italien fut sans doute considéré comme approprié au littoral méridional que longeait la ligne, une Riviera anglaise ${ }^{115}$ (fig. $\mathbf{n}^{\circ}$ 32). 


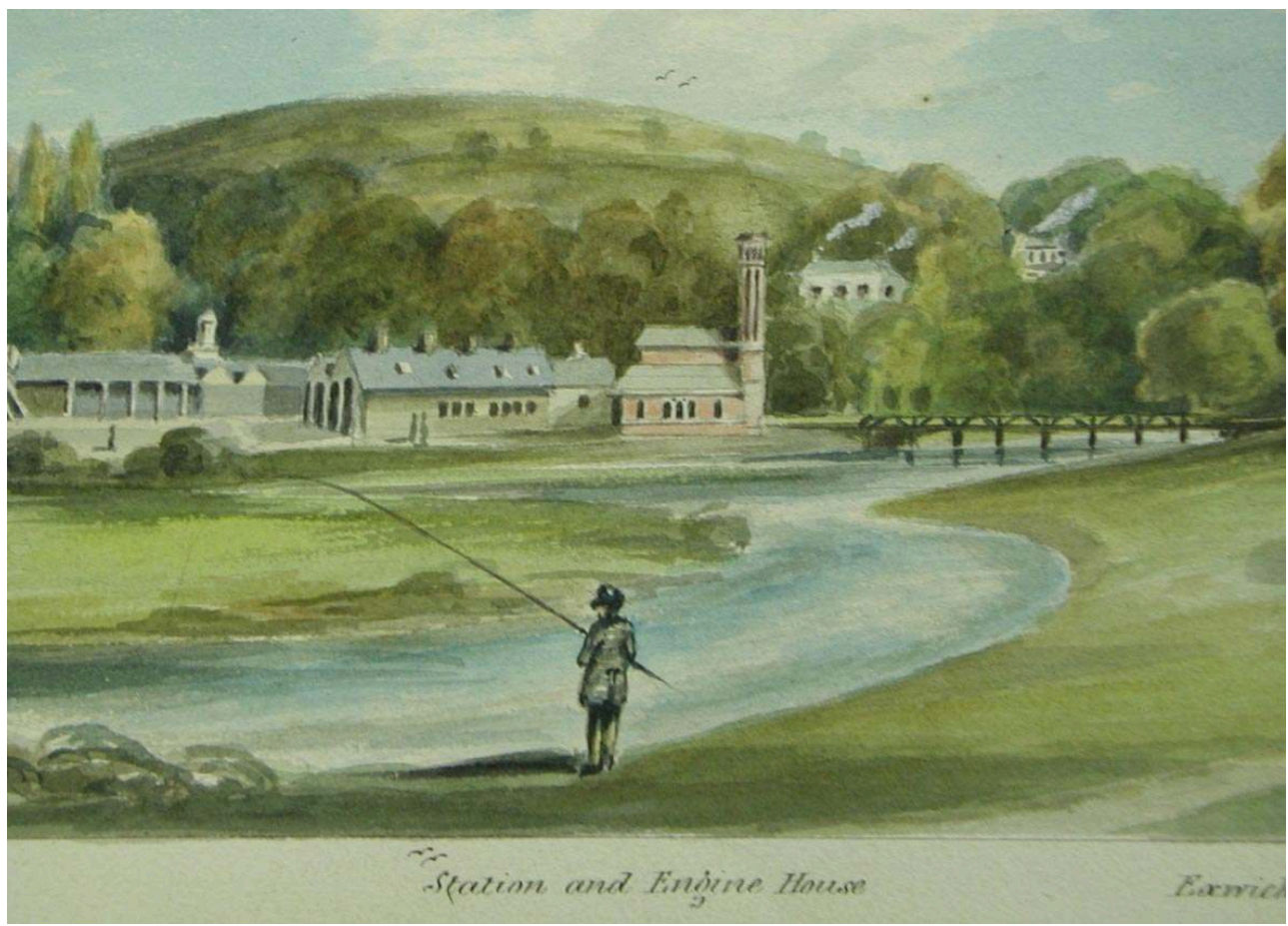

La gare et le bâtiment des machines à Exeter. Détail d'une aquarelle de William Dawson, réalisée en 1847. Album conservé par l'Institution of Civil Engineers, London.

(C) Institution of Civil Engineers.

Adoptant le grand écartement propre au Great Western et à ses compagnies subsidiaires, la ligne était essentiellement à voie unique mais avec quelques sections où la voie et le tube se dédoublaient. Dans un premier temps, et en fonction des différentes pentes à grimper le long du parcours, Brunel envisageait pour ce tube de propulsion des diamètres variables, de 13,15 ou 22 pouces de diamètre $(33,38$ et $56 \mathrm{~cm})$, ce qui aurait nécessité soit de fréquents changements de piston, soit un piston à expansion, s'adaptant à ces changements de format au fur et à mesure du voyage. En fin de compte, un tube d'un seul diamètre (15 pouces) fut installé mais sa livraison, par sections de dix pieds (trois mètres), fut retardée en raison des difficultés rencontrées dans la fabrication : à la différence du tube utilisé sur la ligne irlandaise, fondu en cylindres complets dans lesquels la fente longitudinale fut ensuite usinée par une machine à planer, les tubes du South Devon furent fondus avec la fente déjà incorporée. En attendant la mise en place de ce tube, un service utilisant des locomotives louées à la Great Western fut inauguré au mois de mai 1846, entre Exeter et Teignmouth. Les premiers essais atmosphériques eurent lieu en février 1846 mais ce n'est qu'à la fin du mois d'août 1847 que les premiers services entièrement atmosphériques furent ouverts au public. Certains crièrent victoire, le Besley's Exeter News, par exemple, cité par le Mechanics' Magazine du 28 août 1847, notant que le voyage d'Exeter à Teignmouth était accompli en 42 minutes, avec des arrêts à Starcross et Dawlish : « la distance fut avalée avec la facilité et la précision la plus totale. Tous les services seront atmosphériques d'ici quelques jours. Et tant pis pour les prophètes de malheur!» En novembre, les trains atmosphériques furent aspirés sur tout le parcours de vingt milles $(32 \mathrm{~km})$ entre Exeter et Newton. Le chemin de fer ici était sensiblement de niveau, suivant le bord de la mer. «Il est impossible de suivre une route plus pittoresque et plus sauvage; en 
quelques points, la vague de la mer agitée doit mouiller les impériales des wagons ${ }^{116}$.» Le premier train, avec un wagon-poste, partait d'Exeter à $4 \mathrm{~h}$ du matin; le dernier, un train de marchandises (souvent des poissons), ne revenait de Newton qu'à minuit. Il y avait quatre trains par jour dans un sens et trois dans l'autre. Aux stations intermédiaires, les trains redémarraient par propulsion atmosphérique grâce à des tubes auxiliaires. Mais, très rapidement, sous les effets de ce trafic soutenu, le système commença à montrer les faiblesses qui avaient déjà conduit à son abandon, quatre mois auparavant, à Croydon. Les machines fixes, trop peu puissantes, furent surmenées, devant donner jusqu'à 30 coups de piston par minute au lieu des 18 initialement prévus $^{117}$. Des retards dans l'installation du télégraphe électrique, opérationnel seulement en août $1848^{118}$, exacerbaient ce surmenage et la surconsommation concomitante de houille, car ces machines devaient marcher selon l'indicateur d'horaires imprimé. Les chauffeurs ne pouvaient être avertis d'éventuels retards dans le départ des convois, fréquents à Exeter en raison de l'arrivée tardive des trains en provenance de Bath. Mais les machines fixes devaient surtout lutter contre les entrées d'air de plus en plus sérieuses dans la soupape longitudinale. Au cours de l'hiver 1847-1848, le cuir, imbibé d'eau, gelait ; ensuite, pendant les chaleurs des mois de mai et de juin, desséché par les effets conjugués du soleil et du vide dans le tube, le cuir devint perméable et commença à se déchirer, le climat salin accélérant la corrosion autour des rivets attachant ce cuir au tube. L'eau accumulée dans le tube obstruait le passage du piston. On raconte aussi - selon Buchanan il s'agirait d'une légende locale ${ }^{119}$ - que des rats rongeaient le cuir, trouvant à leur goût la composition d'étanchéité, mélange apparemment enrichi, sur le South Devon, d'huile de foie de morue ${ }^{120}$. Malgré le remplacement par Samuda de sections entières de la soupape, représentant au total presque deux milles et demi, les directeurs de la compagnie, excédés par les retards dans un premier temps, Brunel avait annoncé l'ouverture de la ligne pour l'été 1845 - et par les incessants dépassements du budget ${ }^{121}$, prirent d'abord, en février 1848 , la décision de renoncer à l'application du système atmosphérique au-delà de Totnes, où des bâtiments de pompage étaient pourtant déjà construits et des machines installées (fig. $\mathbf{n}^{\circ}$ 33). 


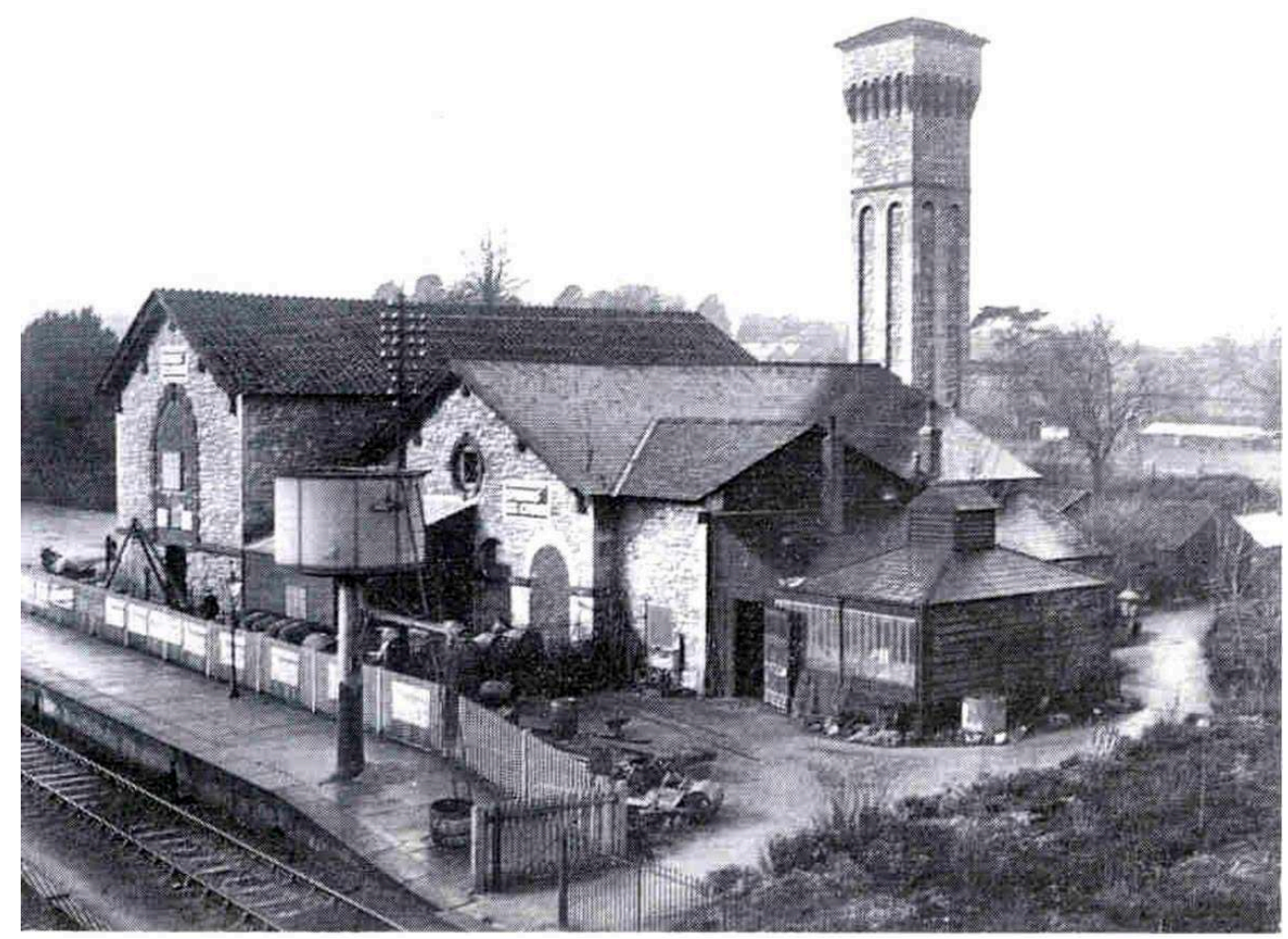

Le bâtiment des machines à Totnes, photo prise dans les années 1960, reproduite dans HADFIELD, Charles. Atmospheric Railways, a Victorian Adventure in Silent Speed, 1967, p. 145.

Puis enfin, le 29 août 1848, ils annoncèrent la suspension totale et définitive de la propulsion atmosphérique : l'ensemble de la ligne serait dorénavant exploité avec des locomotives ${ }^{122}$. Joseph Samuda s'entendit traiter de "visionnaire enragé ${ }^{123}$ » et Brunel dut renoncer à ses honoraires ${ }^{124}$. Thomas Gill, fidèle, en dépit de tout, au système atmosphérique, donna sa démission comme président du conseil d'administration, pensant que la période d'essai n'avait pas été assez longue pour fonder un jugement serein, croyant encore au succès éventuel du système et regrettant la perte du très grand investissement qu'il représentait ${ }^{125}$.

\section{Intérêt français}

Cet arrêt définitif du système atmosphérique dans le comté de Devon laissait encore en activité la petite ligne irlandaise, atmosphériquement exploitée jusqu'en avril 1854, ainsi que l'unique version française d'un chemin de fer atmosphérique, ouverte entre le bois du Vésinet et Saint-Germain-en-Laye en avril 1847. 
Figure 34

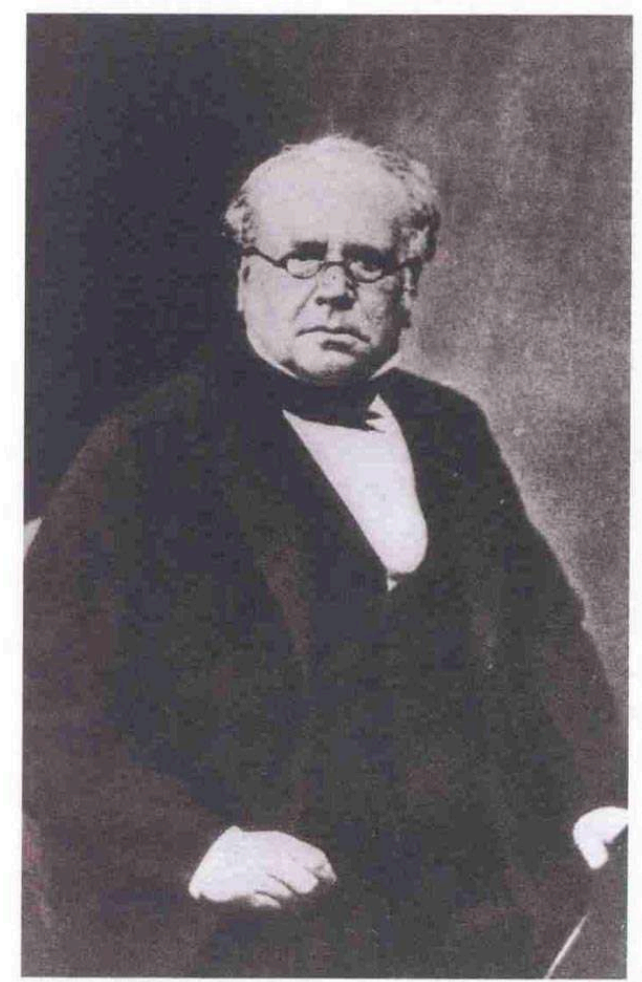

Émile Vuigner, portrait photographique reproduit dans PHILIPP, Elisabeth. HISTOIRE D'UNE ENTREPRISE DE SON TEMPS, LA COMPAgNiE des ENTREPôtS ET MAgASINS GÉNÉRAUX DE PARIS, 2000, p. 46. Repro Paul Smith, 2008.

(C) Paul Smith.

Pour apprécier l'histoire de cette dernière ligne, construite en extension de celle de Saint-Germain ouverte jusqu'au Pecq en août 1837, il faut remonter de nouveau aux premiers essais atmosphériques entre Kingstown et Dalkey à partir de l'automne 1843. À cette époque, parmi les nombreux ingénieurs qui vinrent visiter la nouvelle merveille ferroviaire, on compta plusieurs Français. Arrivé trop tard pour voir les expériences à Wormwood Scrubs, E. Hérard, l'élève-ingénieur des Ponts et Chaussées dont l'article a été cité, fit le voyage en Irlande où, malheureusement pour lui, les travaux sur la nouvelle ligne n'avaient pas encore démarré, la compagnie attendant encore l'arrivée à Dublin des Samuda. Émile Vuigner (fig. $\mathbf{n}^{\circ}$ 34), que nous avons déjà vu associé aux travaux de fondation du pont de Kehl, et que nous recroiserons bientôt à Saint-Ouen, eut davantage de chance ou s'était mieux préparé, faisant le voyage en Irlande, au début de l'année 1844, en compagnie de l'un des inventeurs du système (sans doute l'un des frères Samuda, mais il ne précise pas lequel). Ingénieur autodidacte nommé directeur du service de la voie du chemin de fer de l'Est à partir de juillet 1847, Vuigner travaillait alors comme directeur d'exploitation et d'entretien de la compagnie concessionnaire des canaux de l'Ourcq et de Saint-Denis, appartenant à Georges Tom Hainguerlot, dans laquelle il était entré en 1818. C'est à l'instigation de cette importante compagnie privée que Vuigner accomplit sa mission d'étude à Kingstown, en vue d'une possible application du système atmosphérique sur un chemin de fer envisagé entre Paris et Meaux, le long de l'un des chemins de halage du canal ${ }^{126}$. Vuigner revient en France entièrement convaincu par ce qu'il avait pu voir ${ }^{127}$, publiant un mémoire dans lequel il décrit le fonctionnement du système et fait le catalogue de 
ses avantages acquis en matière de sécurité et d'économie d'installation. Il note par exemple la légèreté des rails que le nouveau système autorise, et rappelle qu'en douze années de fonctionnement, les rails du Liverpool-Manchester avaient déjà dû être renouvelés trois fois. Il insiste surtout sur la supériorité du système pour la montée de ce que les Anglais appelaient «bad gradients », de « mauvaises pentes » de 1,5 et même $2 \mathrm{~cm}$ par mètre, inadmissibles dans "l'ancien système", et pour la négociation à grande vitesse de courbes ayant un rayon de moins de $500 \mathrm{~m}$, également interdites aux locomotives françaises. En effet, à l'époque, comme nous l'avons vu, l'administration des Ponts et Chaussées n'admettait pas dans le tracé des chemins de fer des pentes supérieures à cinq millimètres par mètre, ni des courbes inférieures à mille mètres ou, tout au plus, à 500 ou $600 \mathrm{~m}$ à l'approche des gares. La zigzagante ligne de Dalkey comprenait quant à elle des courbes successives, sans raccordements rectilignes, de seulement 173 et $231 \mathrm{~m}$ de rayon, ainsi que des rampes de $1,75 \mathrm{~cm}$ par mètre. Vuigner ne prétendait pas pour autant que le système atmosphérique était arrivé à un « degré complet de perfectionnement », rendant compte, justement, des dernières améliorations techniques apportées par les Samuda: un boulon en bois utilisé dans l'assemblage de la tige reliant le wagon directeur au châssis du piston, boulon qui se romprait sous l'effet d'un choc violent et éviterait - ce que redoutaient certains - que le piston ne parte comme un boulet pour jeter le désordre dans la prochaine station; ou encore, un dispositif (des trous) manœuvré depuis l'intérieur du wagon directeur pour laisser passer l'air à pression atmosphérique devant le piston, agissant ainsi comme un frein. Mais pour Vuigner, malgré de tels perfectionnements en cours, le chemin de Kingstown à Dalkey n'était pas à considérer comme une expérience. Hormis son mode de propulsion innovateur, c'était un chemin de fer commercial ordinaire, comptant 42 trains par jour; le jour de Noël 1844, selon les registres de la compagnie, il avait transporté plus de 10000 voyageurs en cinq heures! «La question de l'avenir et de l'utilité du système atmosphérique ne fait plus de doute aujourd'hui », écrivit-il, en se demandant si, de gaîté de cœur, des ingénieurs aussi distingués que Vignolles (sic), Brunel et Cubit (sic) engageraient les capitaux de leurs clients, et leur propre responsabilité et renommée, s'ils entretenaient le moindre doute quant à cet avenir.

Pour Vuigner, et pour le chemin d'une quarantaine de kilomètres le long du canal de l'Ourcq comportant un tracé très sinueux entre Claye et Meaux, l'idéal serait d'associer le système atmosphérique à l'irlandaise avec le système, français, des trains articulés, inventé par Jean-Claude Républicain Arnoux. Né en 1792, cet ingénieur polytechnicien de la promotion de 1811, chef des ateliers de construction des messageries Laffitte, Caillard et Compagnie, avait breveté, le 20 mars 1838, un système de wagons articulés dont les essieux mobiles, pivotant sur chevilles ouvrières, restaient placées normalement à la voie, les roues tournant librement sur des fusées. La position des essieux était commandée par les courbes mêmes de la voie, le premier essieu du train étant équipé d'un appareil directeur orienté par quatre galets obliques, en contact avec les bords intérieurs des rails (fig. $\mathbf{n}^{\circ}$ 35). Cette invention cherchait, justement, à autoriser les voies à courbures de court rayon, permettant à leur tracé de contourner des obstacles naturels ou fonciers, et le dispensant ainsi, comme devait le faire aussi le système atmosphérique, de réaliser de coûteux travaux d'art. Après des essais en grandeur réelle sur un chemin en circuit fermé de $1142 \mathrm{~m}$ de long établi à SaintMandé, Arnoux, en 1840, avait déjà proposé d'appliquer son invention à une ligne suivant les berges du canal de l'Ourcq jusqu'à Meaux. Suite à un rapport présenté par François Arago, une concession officialisée par la loi du 5 août 1844 l'autorisa à 
construire une ligne expérimentale, mais sans subvention de l'État. Longue de 10,6 km, elle joignait Paris à Sceaux et ses attractions - son marché et un célèbre bal - à partir d'un embarcadère construit à la barrière d'Enfer sur le territoire de la commune de Montrouge, l'actuelle station de métro et de RER de Denfert-Rochereau (fig. $\mathbf{n}^{\circ} \mathbf{3 6}$ ). Mais, en fin de compte, ce "chemin d'expérience» n'utilisait pas la propulsion atmosphérique, expérimentée à la même époque sur le Paris-Saint-Germain ${ }^{128}$.

Figure 35

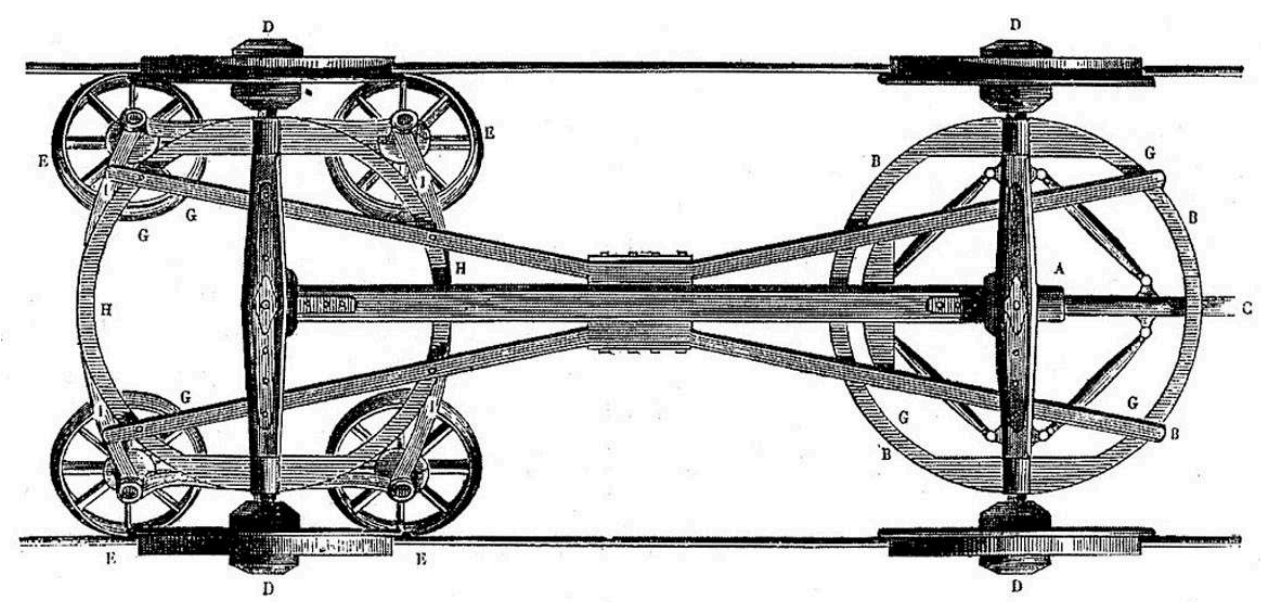

Le système des trains articulés de Jean-Claude Républicain Arnoux. Reproduit dans LANGLOIS, GillesAntoine. MONTPARNASSE Et LE XIVE ARRONDISSEMENT, 2000, p. 107. Repro Paul Smith, 2008.

(c) Paul Smith.

Figure 36

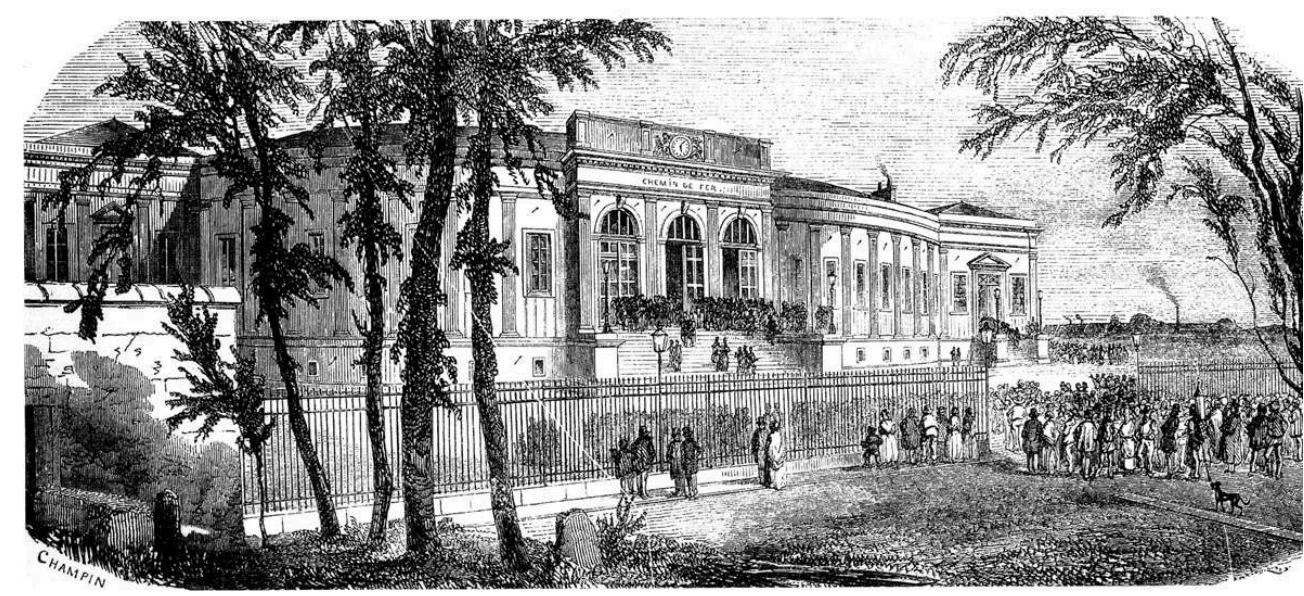

L'embarcadère de la ligne de Sceaux, l'actuelle gare Denfert-Rochereau. L'ILLUSTRATION, 6 juin 1846. Repro Paul Smith, 2008.

(C) Paul Smith.

62 Prenant vers 1840 la succession de son frère, le saint-simonien Stéphane Mony, Eugène Flachat (fig. $\mathbf{n}^{\circ} \mathbf{3 7}$ ), l'ingénieur de cette dernière ligne ${ }^{129}$ et bientôt chargé de la réalisation de son extension atmosphérique, figure également parmi les Français ayant fait le voyage en Irlande. 


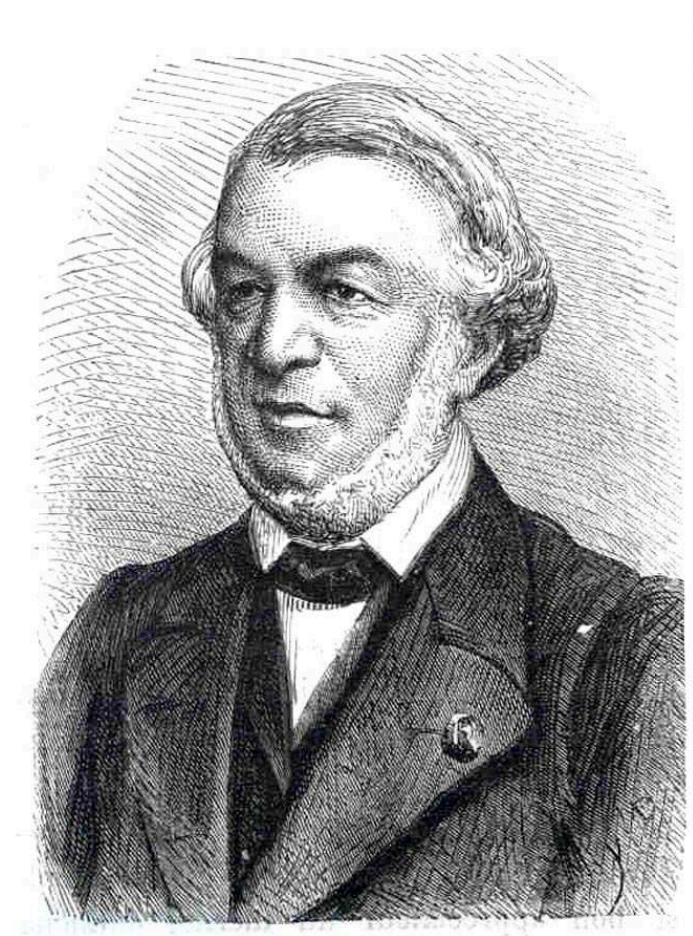

Fig. 140. - Eugène Flachat.

Eugène Flachat, portrait dans FIGUIER, Louis. Locomotive et chemins de fer. LES MERVEILLES DE LA SCIENCE OU DESCRIPTION POPULAIRE DES INVENTIONS MODERNES, 1867, p. 301. Repro Paul Smith, 2008.

(c) Paul Smith.

63 Un rapport lu par Émile Pereire à l'assemblée générale extraordinaire de la compagnie de Saint-Germain du 8 novembre 1844, informe les actionnaires que leur sousdirecteur, Isaac Pereire, « accompagné de M. Eugène Flachat, ingénieur, a été passer un mois en Angleterre et en Irlande, pour visiter le chemin atmosphérique de Dalkey et pour étudier les nouveaux travaux de même nature qui sont en cours d'exécution dans ce pays ; les plus grandes facilités leur ont été partout données avec une libéralité que nous proclamons ici avec une vive reconnaissance; partout ils ont trouvé un empressement bienveillant à leur fournir les renseignements dont ils ont pu avoir besoin. » Émile Pereire signale tout spécialement ici l'administration du chemin de Kingstown à Dalkey, M. Brunel fils et $\mathrm{M}$. Cubitt, « ingénieurs éminents dont le nom a acquis une grande autorité en Angleterre et sur le continent ${ }^{130} »$.

À côté de ces visites d'ordre privé, le Conseil des Ponts et Chaussées au ministère des Travaux publics envoya également en Irlande un spécialiste pour faire une enquête particulière et un rapport officiel sur le système. Il ne s'agissait pas, cette fois-ci, de Teisserenc de Bort - quoique ce dernier accompagnât le ministre, Pierre-Sylvain Dumon, lors d'une visite au chemin de Croydon en septembre $1846^{131}$ - mais de l'inspecteur divisionnaire adjoint, Jacques Mallet. Né à Dieppe en 1787, celui-ci avait acquis une certaine expérience ferroviaire en tant qu'ingénieur en chef des Ponts et Chaussées dans le département de la Seine-Inférieure, notamment dans les études entreprises à partir de 1830 relatives au chemin de fer de Paris à Rouen, au Havre et à Dieppe $^{132}$. En mars 1839, il avait été nommé ingénieur en chef directeur du service du port du Havre, où il a peut-être pu voir les essais du système atmosphérique menés par 
James Bonfil dans les ateliers des frères Nillus. Au début du mois de novembre 1843, Mallet reçut du sous-secrétaire d'État, Alexis Legrand, le courrier suivant :

"L'ouverture du chemin de fer atmosphérique de Kingstown à Dalkey va avoir lieu dans quelques jours. Un des principaux actionnaires de la compagnie qui va exploiter ce chemin m'a fait connaître que si l'administration croyait devoir envoyer sur les lieux un ingénieur français avant l'ouverture du chemin, la compagnie le mettrait de se [illisible] aux faits et aux études qu'il jugerait convenables. J'ai pensé, Monsieur, qu'il y avait une utilité d'accepter cette offre dans une circonstance aussi digne d'intérêt et j'ai l'honneur de vous annoncer que sur ma proposition, M. le Ministre des Travaux Publics ${ }^{133}$ vous a désigné pour aller en Irlande visiter le chemin de fer atmosphérique. » L'actionnaire ayant averti l'administration française de l'ouverture imminente de la ligne était, bien sûr, James Bonfil, demeurant à Paris, 1 rue Caumartin, avec qui Mallet devait se mettre aussitôt en rapport pour l'organisation de son voyage. «Je vous invite à partir immédiatement, poursuivait Legrand. Vous voudrez bien étudier avec soin tous les détails du système nouveau. Vous chercherez à vous rendre compte des frais de premier établissement, des frais d'entretien et d'exploitation. Vous comparerez ces frais avec ceux que comporte le système usité aujourd'hui. Vous examinerez si dans le cas où le nouveau mode de propulsion aurait des chances de succès, il serait possible de l'appliquer aux chemins de fer déjà exécutés. Vous ne négligerez en un mot aucun moyen de recueillir des notions exactes et précises sur les divers points qui pourraient éclairer l'administration et fixer son opinion sur les mérites de cette invention au point de vue pratique ${ }^{134}$."

Mallet se trouvait à Dalkey les 12, 13 et 14 novembre, accompagné par Joseph Samuda et James Bonfil, un «zélé et intelligent interprète». James Pim, qui, selon Mallet, jouissait à Dublin « d'une considération grande et méritée ", veillait, quant à lui, « à ce que rien ne me manquât pour les expériences.» Faites par beau temps (fait effectivement digne d'être noté), celles-ci consistaient essentiellement dans la mesure du temps nécessaire pour faire le vide dans le tube, la mesure du temps de montée pour des convois de poids différents (un maximum de 45 milles à l'heure, ou $72 \mathrm{~km} / \mathrm{h}$, fut enregistré pour un convoi de 38 tonnes au total, comportant sept voitures avec 200 passagers) et la mesure, enfin, du temps de descente par gravité - environ cinq minutes - avec le piston rangé de côté, « ce qui se fait avec la plus grande facilité ». Pendant les trois jours de son séjour, le chemin atmosphérique «n'a pas cessé d'être en activité. Une foule de peuple envahissait les wagons et un grand nombre de personnes de la haute société de Dublin avait été attiré par la curiosité (...) Pendant ce temps, il n'est pas arrivé le moindre accident. Sur un pareil chemin, la sécurité peut être regardée comme complète ${ }^{135}$.» Il semblerait qu'au cours de ses investigations, Mallet ait croisé Brunel $^{136}$. Le 14 novembre, il fut invité à un dîner par le maire de Dublin ${ }^{137}$. À Londres, il put rencontrer aussi Jacob Samuda dans ses ateliers à Southwark - il lui reconnaissait " un esprit remarquable et une grande loyauté de caractère » - pour converser avec lui de l'application du système atmosphérique à une longue ligne, et pour examiner des parties de la soupape utilisée à Wormwood Scrubs, encore susceptibles de servir, selon Mallet, malgré plusieurs années d'exposition à l'air et à la pluie.

Qu'il ait été charmé par la qualité de son accueil en Irlande et le beau temps qui y régnait, séduit par les explications technico-commerciales des Samuda, impressionné par les avis favorables du célèbre Brunel ou tout simplement convaincu par le fonctionnement efficace de la ligne, transportant des foules de curieux, Mallet, comme 
Teisserenc avant lui et Vuigner peu après, rentra en France complètement gagné au système ${ }^{138}$. À la suite d'un bref rapport préliminaire, diffusé dès son retour fin novembre, il rédigea un rapport plus détaillé, apportant des réponses étayées aux questions posées par Legrand dans sa lettre de mission. Daté du 10 janvier 1844, ce rapport fut publié in extenso par le Journal des Chemins de Fer dans ses numéros des 2, 9 et 16 mars, une traduction en langue anglaise paraissant peu après dans les Quarterly Papers on Engineering ${ }^{139}$.

Après un rappel des antécédents de l'invention de Clegg et Samuda - il mentionne Papin, Medhurst (qualifié d'ingénieur danois ${ }^{140}$ ), Valance (sic) et Pinkus -, Mallet explique le principe de la propulsion atmosphérique et décrit les conditions de son application sur la ligne de Dalkey, ainsi que les expériences qu'il avait pu y mener luimême. Sa seule critique à l'égard du système concerne le "heater", le chauffoir attaché à l'arrière du wagon conducteur et destiné à liquéfier la composition d'étanchéité. Même à de faibles vitesses, ce chauffoir, d'un mètre de longueur, ne serait en contact avec la soupape que pendant un dixième de seconde et ne serait d'aucun effet pratique. Lors des arrêts, en revanche, il stationnerait plus longtemps sur la soupape et pourrait l'endommager ou, du moins, faire couler la composition dans le tube. Sur les lignes de Croydon et du South Devon, comme sur la ligne française, le chauffoir fut effectivement abandonné, la soupape étant refermée par la seule pression d'une roulette en fer. Après la description de la ligne irlandaise, Mallet analyse, dans les chapitres suivants, les avantages d'une application plus générale de la propulsion atmosphérique sur les chemins de fer, avantages en terme de sécurité surtout - «ce système, n'admettant pas de locomotives, est exempt des dangers auxquels elles donnent lieu "-, mais aussi en regard des économies de première installation et d'exploitation. Ces avantages, conclut son rapport, étaient assez manifestes pour motiver un essai du système en France.

La diffusion du rapport Mallet fit sortir le débat des seuls cénacles des ingénieurs des chemins de fer. Au cours des années 1844 et 1845, L'Illustration revint à plusieurs reprises sur le système atmosphérique, tenant ses lecteurs au courant du triomphe de la ligne irlandaise et rendant compte d'autres systèmes atmosphériques dus à des inventeurs français. Dans son numéro du 25 mars 1844, le grand quotidien sérieux de l'époque, le Journal des Débats, loua le rapport Mallet pour sa "sagacité remarquable » et reprit ses calculs comparatifs pour les frais d'établissement du «système actuel » (qui prenaient pour base les dépenses faites pour les chemins de fer de Rouen, d'Orléans et de Montpellier à Nîmes) et ceux du système atmosphérique en Irlande : $248700 \mathrm{~F}$ par kilomètre pour le premier et $213600 \mathrm{~F}$ pour le second. Pour le transport des voyageurs l'économie serait dans le rapport de trois à cinq.

Comme en Angleterre cependant, tous les spécialistes, en particulier des hommes ayant une expérience pratique de la gestion d'un chemin de fer, n'étaient pas convaincus par les promesses du nouveau système. Jules Petiet ${ }^{141}$, par exemple, sorti major en 1832 de la première promotion de l'École centrale des arts et métiers, passé ensuite, de 1835 à 1842, par le bureau d'ingénieurs civils créé par Eugène Flachat ${ }^{142}$, et chargé à partir de 1842, peu après l'accident de Meudon, de l'exploitation de la ligne Paris-Versailles (Rive gauche), faisait publiquement état de ses doutes. Dans une série de lettres adressées au Journal des Chemins de Fer à partir du 6 janvier 1844, Petiet alignait de nombreux arguments contre le système, "tellement cher de premier établissement et tellement cher d'entretien même qu'il ne pouvait pas supporter la comparaison avec les moteurs 
à locomotives (...) Toute application de ce genre serait folie.» Il se chargea de la critique détaillée, point par point, du rapport Mallet, observant pour commencer que ce dernier, séduit par le système, s'en était fait le partisan au lieu d'en rester le juge. Pour Petiet, Samuda était un charlatan. Le 6 janvier 1844, le Journal publia également un courrier envoyé par un " pauvre conducteur de machines locomotives menacé dans son industrie par suite de l'engouement qui s'attache à l'apparition du système de railway atmosphérique. » Avec une aisance qui peut surprendre de la part d'un mécanicien, cet auteur anonyme fournit d'autres arguments pratiques contre l'application du système sur une grande ligne : les difficultés dans le service des stations ; l'impossibilité pour les trains atmosphériques de reculer; le fait qu'ils dépendent trop des freins (pour ralentir, les locomotives ne comptaient que peu sur les freins, mais renversaient plutôt la vapeur) ; l'interruption de tout service en cas de panne d'une machine fixe: «Si le service est interrompu, pour ramener le train à la station, il faudrait avoir recours à des chevaux, si l'on ne veut pas absolument se servir de nos pauvres machines locomotives. »

71 À Arras (dont nous verrons bientôt les liens particuliers avec les initiatives atmosphériques), le colonel du Génie Claude-François Répécaud démonta les prétendus avantages économiques du système basés sur le principe d'une voie unique avec gares d'évitement pour le croisement des convois ${ }^{143}$. Le nouveau système, avec son coussin d'air dans le tube, empêcherait certes toute collision mais rendrait les mouvements de ces convois extrêmement difficiles à coordonner. Si, par exemple, deux convois se présentaient simultanément aux deux extrémités de la même section du tube sous vide, l'un des deux serait obligé de reculer jusqu'à la gare précédente, qui serait peut-être déjà occupée. Pour ce faire, d'ailleurs, le wagon-conducteur devrait être remis en tête de son convoi: "pendant que se ferait cette longue et pénible manœuvre, tout mouvement serait forcément interrompu dans toute l'étendue du chemin; si ce n'est pour les convois qui s'éloigneraient du point de concours, tous les autres devraient faire halte dans les gares; il y aurait donc une grande perturbation dans le service de ce chemin, et la cause pourrait s'en renouveler fréquemment, si, comme on l'a supposé, le chemin était très fréquenté. » Répécaud contestait aussi les économies supposées en matière de travaux d'art, estimées par Mallet à $800000 \mathrm{~F}$ par kilomètre, une "exagération évidente ». Sur de fortes pentes, le tube de $38 \mathrm{~cm}$ de diamètre utilisé en Irlande ne donnerait pas assez de force. Un tube de plus grand diamètre pourrait être employé sur certaines sections difficiles d'une ligne, mais, outre l'accroissement des dépenses en fonte, une telle variation de diamètres demanderait des changements de piston; le vide plus important sur ces sections à rampe s'étendrait aussi aux sections à niveau, créant une accélération incontrôlée des convois. Ainsi, selon, Répécaud, «le système de propulsion atmosphérique ne doit pas être substitué à celui des locomotives, parce que le premier, appliqué à un chemin à une seule voie, présenterait de graves inconvénients et coûterait plus que le deuxième appliqué à un chemin à double voie ; et si, pour éviter ces inconvénients, on doublait la voie, l'augmentation de la dépense, comparativement à ce que coûtent les chemins à locomotives, serait telle que les partisans les plus prononcés des chemins atmosphériques y renonceraient forcément ${ }^{144}$.»

72 En même temps que Répécaud faisait ces observations devant la Société académique d'Arras, dans ses séances du 19 juillet et du 2 août 1844, les conclusions similaires de Robert Stephenson sur le peu de viabilité du système commençaient à être connues en France. Le rapport de Stephenson fut intégralement traduit en langue française par 
Alphonse Lauvray et publié, comme nous l'avons vu, par le Journal des Chemins de Fer, en juillet 1844. En août, Mallet lui-même communiqua l'essentiel des conclusions de Stephenson à la Société d'Encouragement pour l'Industrie nationale. Un membre de la société fit observer, toutefois, que ces conclusions n'étaient pas suffisantes en ellesmêmes pour faire apprécier les avantages ou les inconvénients du système, et qu'une étude plus approfondie, ainsi qu'une plus longue pratique seraient nécessaires pour porter sur le système un jugement définitif ${ }^{145}$.

\section{Inventions françaises : Andraud, Arnollet, Hédiard, Hallette...}

À cette époque, en effet, en France, et pas seulement à Paris, les chemins de fer atmosphériques suscitaient plutôt l'enthousiasme, voire même l'engouement que redoutait le pauvre conducteur de locomotives, et qui semblait bien prévaloir outreManche. Entre 1844 et 1846, selon le recensement de Charles Hadfield, près de 80 projets de chemins de fer atmosphériques virent le jour au Royaume-Uni, et deux autres en Jamaïque ${ }^{146}$. En France, le système atmosphérique fut préconisé par François Arago, en juillet 1844, pour une partie du chemin de fer de Paris à Strasbourg ${ }^{147}$, tandis que pour la ligne de Dijon à Genève et à Lausanne, passant par le Jura, des fortes pentes de deux à quatre centimètres par mètre semblaient également commander, selon l'ingénieur Antoine-Rémy Polonceau dans une étude de février 1845, le recours à un système atmosphérique, celui de Monsieur Andraud ayant ses préférences ${ }^{148}$.

\section{Antoine Andraud}

Les différentes inventions d'Antoine Andraud ne relevaient pas, en fait, du système atmosphérique proprement dit, étant fondées sur le principe non pas de l'air raréfié et de la pression atmosphérique, mais sur celui de l'air comprimé. Reprenant, sans doute sans le savoir, une idée esquissée dans les premiers brevets de Medhurst, Andraud réfléchissait au cours des années $1830^{149}$ à une manière d'exploiter l'énergie de l'air en le comprimant dans des récipients ou des "vases " qui pouvaient être transportés comme réserves de force motrice. La lecture de ses premiers textes, de 1839 et de $1840^{150}$, publiés «sous la pression de la grande question des chemins de fer », le fait apparaître comme un théoricien visionnaire de l'air comprimé. Pour Andraud, l'air comprimé était une énergie dont la force était destinée à changer la face du monde matériel et, par suite, du monde moral. C'était un signe représentatif de toutes les forces, comme l'argent était un signe créé par le commerce pour représenter toutes les valeurs. La production de l'air comprimé, par la marche des eaux ou la course de vents, était pour ainsi dire gratuite : l'air est donné par la nature, et avec profusion. Ainsi proposait-il d'établir partout où il le faudrait des roues "éoliques » et hydrauliques adaptées à des pompes foulantes qui comprimeraient l'air dans des récipients. "Les autorités municipales établiront dans les villes de vastes réservoirs d'air comprimé où tout le monde ira, pour les menus besoins domestiques, puiser de la force, devenue objet d'utilité première, comme on va aujourd'hui puiser de l'eau à nos fontaines publiques. "

Dans les différentes anticipations " aérodynamiques » d'Andraud, cette nouvelle force s'appliquerait tout d'abord aux machines fixes : un moteur gratuit trouverait sa place 
chez tous les artisans où on avait recours à la force brute, tels que tourneurs, menuisiers, potiers, etc: "je laisse aux mécaniciens le soin d'étudier les meilleurs moyens d'application ; ce sont affaires de détail. » L'air comprimé s'appliquerait, bien sûr, aux chemins de fer, mais aussi à la locomotion sur voies ordinaires : on aurait des récipients de force en magasin comme on a des chevaux dans l'écurie. Il s'appliquerait à la navigation fluviale, ainsi qu'à l'agriculture, pour le labourage des terres, le battage des grains et le charriage des récoltes. Par des canons à air comprimé, il servirait à la défense des villes de guerre. Il serait utilisé pour la perforation de la terre : «il nous sera possible d'aller ouvrir le passage à des eaux souterraines qui jailliront bouillantes à la surface et viendront en aide à nos diverses industries ${ }^{151}$." Par des conduites souterraines hermétiquement fermées, l'air comprimé pourrait envoyer des lettres contenues dans des cylindres: "on pourra écrire de Paris à Marseille et recevoir la réponse dans la même journée. » L'air comprimé s'appliquerait à la navigation aérienne d'un oiseau artificiel : un récipient chargé à soixante atmosphères pouvait donner cinq mille coups de piston, c'est-à-dire cinq mille battements d'aile. Il s'appliquerait enfin à la construction d'orgues gigantesques, d'immenses harmonies donnant « des concertscolosses qui domineraient les cités comme les roulements sublimes du tonnerre ${ }^{152}$. »

À notre époque, sensibilisée au besoin d'énergies renouvelables, certains propos d'Andraud, formant la conclusion de son texte de 1840 et repris dans une autre publication de $1846^{153}$, paraissent particulièrement prémonitoires. "Sur quelle base, demande-t-il, est assise aujourd'hui l'industrie des peuples? Sur la houille. Mais en aurez-vous toujours? N'est-ce pas là une source de richesse qui doit se tarir en peu d'années? Savez-vous que la nature a mis deux ou trois mille ans à la formation de ces couches de charbon que vous brûlez en quelques années? Que sera-ce donc lorsque toutes nos villes s'éclaireront au gaz comme Paris, lorsque nous aurons couvert notre territoire d'un réseau de chemins de fer ? [...] L'intention manifeste de la providence était que l'homme trouvât un jour toutes les puissances mécaniques nécessaires à ses besoins dans les immenses et intarissables réservoirs où il puise la vie, et non dans le déchirement avide des entrailles de la terre, travail impie contre lequel la nature proteste chaque jour par quelque horrible catastrophe.» Doctrinaire exclusif de l'énergie de l'air comprimé, qualifiée d'énergie « de tous les pays et de tous les temps ${ }^{154}$ ", Andraud redoutait l'assimilation de ses propositions au système dit atmosphérique, toujours tributaire de la machine à vapeur pour faire le vide, vide d'ailleurs dont on ne pouvait faire ni accumulation ni réserve: " on doit absolument rejeter le système atmosphérique par le vide dont on fait tant de bruit, parce que ce système, esclave de la vapeur, loin d'apporter une amélioration, ne ferait qu'accroître les difficultés de l'avenir ${ }^{155}$.»

77 Mais Andraud n'était pas qu'un doux rêveur sous influence fouriériste, un sympathique visionnaire aux paroles en l'air. Son mémoire de 1839 attira l'attention d'un autre ingénieur, Tessié du Motay, qui travaillait alors à Cholet à la construction de locomotives fondées sur les mêmes principes. Les deux hommes s'associèrent pour entreprendre une série d'expériences destinées dans un premier temps à trouver la forme et la matière des récipients de l'air comprimé, «la pièce capitale » de leur nouveau système dynamique. Ces expériences eurent lieu dans «l'ancienne fonderie de la pompe à feu de Chaillot », ateliers où les deux inventeurs avaient peut-être pu voir l'échantillon du système atmosphérique de Clegg et Samuda. Utilisant une machine à vapeur de six chevaux mettant en jeu une pompe foulante de grande puissance, et se protégeant, par prudence, derrière un mur épais, Andraud et Tessié expérimentèrent 
des récipients en cuivre, en toile de coton doublée de caoutchouc et repliée six fois sur elle-même, en bois doublé de zinc et garni de bons cercles de fer, et en tôle de fer de deux millimètres d'épaisseur. Ce dernier matériau paraissait le plus convenable; des récipients de cent litres de capacité, de forme cylindrique terminée par deux hémisphères, résistaient constamment à des pressions de trente à trente-cinq atmosphères et ne cédaient qu'à plus de soixante-quinze atmosphères : «les vases ont cédé, mais sans explosion; la tôle se déchire et l'air s'échappe par une fente à peine visible, avec un sifflement très aigu ${ }^{156}$. »

En mai 1840, les deux ingénieurs prirent un brevet d'invention de quinze ans comprenant, d'une part, leur principe nouveau d'une force unique dite air comprimé, susceptible d'être mise en réserve, transportée et dépensée à volonté, soit comme moteur soit comme force de compression, et, d'autre part, l'ensemble des appareils mécaniques destinés à rendre l'air comprimé ou dilaté applicable à toute espèce d'industrie : pompe à effet progressif, récipient à force multiple capable de supporter l'air comprimé à 90 atmosphères, régulateur, dilateur, machines rotatives ou à mouvement de va-et-vient, piston métallique, dynamomètre, fluomètre, presse aérohydraulique, robinet autoclave ${ }^{157}$. Ils se préoccupaient aussi de la conception des turbines éoliques et des roues fluviales, les agents mécaniques appelés à remplacer la machine à vapeur pour opérer gratuitement la compression de l'air ${ }^{158}$. Ils travaillaient enfin à différentes applications de leur énergie: une pompe aéro-hydraulique dans laquelle l'air comprimé, faisant office de piston, pouvait élever l'eau «à des hauteurs illimitées »; un canon à force multiple dans lequel l'air comprimé pouvait dépasser la force de la poudre ; et, objet principal de leurs expériences, une voiture à air comprimé qui pourrait se mouvoir sur un chemin de fer.

Une première voiture d'essai, mesurant trois mètres de long, deux mètres de haut et 1,50 mètre de large, fut lancée pour la première fois sur un petit chemin de fer d'une centaine de mètres de long le 9 juillet 1841, parvenant, deux jours plus tard, à transporter huit voyageurs sur un peu plus d'un kilomètre. Cette voiture comportait sept récipients d'air comprimé dont un récipient spécial contenant de l'air très fortement pressé. Baptisé par ses créateurs «le cheval de montagne », ce dernier était destiné à donner un coup de collier pour franchir une côte. Vu la légèreté de leur locomotive, les inventeurs avaient pensé tout de suite à son usage sur des chemins à forte pente, de 1,5 à $2 \mathrm{~cm}$ par mètre. Une fois sur les rails, la direction de leur voiture se résumait à l'ouverture et la fermeture d'un robinet: « un enfant intelligent peut la conduire. » D'après ces premiers essais, Andraud et Tessié du Motay estimaient à trois ou quatre lieues la distance que pouvait franchir la voiture sans être réapprovisionnée. Pour cela, et dans l'hypothèse d'un service organisé, de vastes réservoirs placés de distance en distance sur le bord des chemins permettraient de renouveler la force motrice en « quelques secondes ${ }^{159}$ ». 
Figure 38

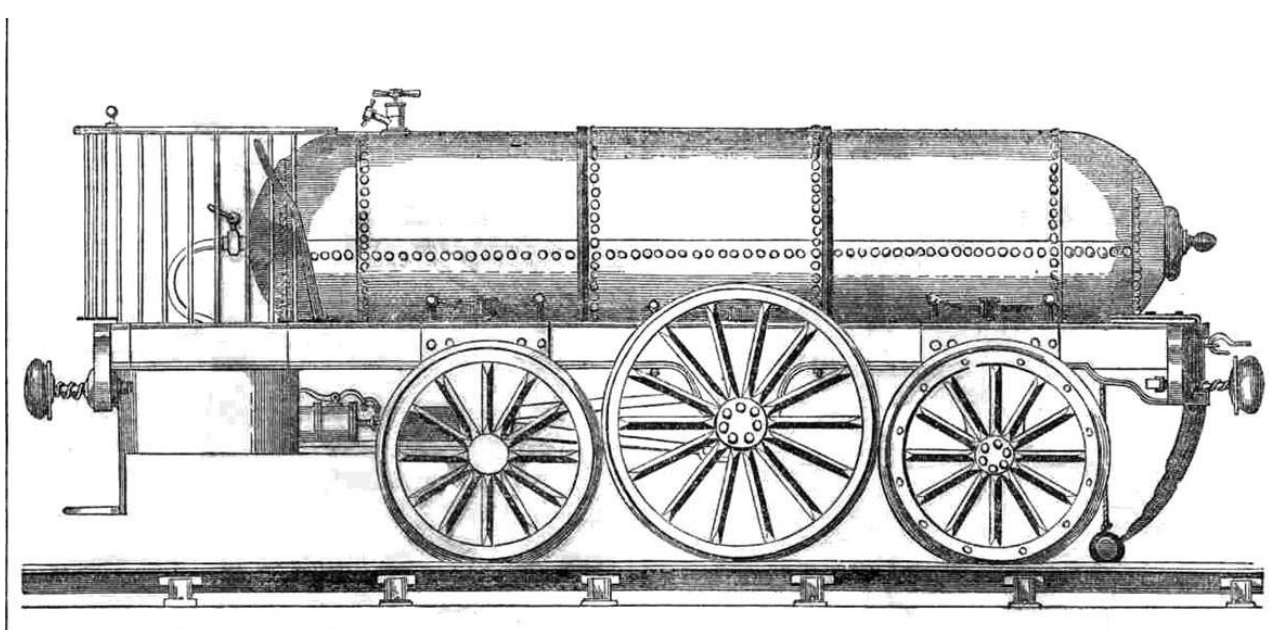

(Fig. 5. - Chemin atmosphérị̣ue. - Locomotive à air comprimé de M. Andraud,)

La locomotive à air comprimé de Monsieur Andraud. L'IlLustration, 2 novembre 1844. Repro Paul Smith, 2008.

(C) Paul Smith.

Le 26 août 1844, Andraud et Tessié du Motay lancèrent sur la ligne de Paris-Versailles (Rive-gauche) une nouvelle « locomotive-waggon » à air comprimé. Pesant maintenant cinq tonnes et ressemblant à une chaudière cylindrique posée sur une plate-forme à six roues, son mécanisme était d'une simplicité extrême. Son aspect ne manquait pas "d'élégance ${ }^{160}$ » (fig. $\mathbf{n}^{\circ}$ 38). Dans ce récipient unique de 3,3 $\mathrm{m}$ cubes, construit en tôle de fer de 13 millimètres d'épaisseur, l'air devait être comprimé de quinze à vingt atmosphères pour faire marcher les pistons des cylindres moteurs montés à l'arrière du véhicule. Avant d'entrer dans les cylindres, il passait par un manchon garni de fer chaud pour opérer une dilatation de l'air comprimé et augmenter sa force. Dans un premier temps, l'administration de la compagnie de la Rive gauche avait mis à la disposition d'Andraud une locomotive à vapeur inoccupée, utilisée pour comprimer l'air. Mais celle-ci ayant été vendue, l'expérimentateur fut réduit à faire marcher ses pompes foulantes à bras d'homme, force à peine suffisante pour obtenir une pression de cinq atmosphères. La voiture parvint néanmoins à faire ses premiers pas avec un certain succès, acquérant une vitesse de six à sept lieues à l'heure et parcourant une distance d'environ un kilomètre. Deux commissaires du gouvernement, Baude et Bineau, eurent le courage de monter sur la machine avec l'inventeur qui la dirigeait. Le 21 septembre un nouvel essai eut lieu, cette fois-ci avec un certain apparat et devant une nombreuse assistance de savants, d'administrateurs et de curieux. Toujours contraint de comprimer l'air à bras, la faible pression (sept atmosphères au lieu de vingt) permit néanmoins au véhicule de parcourir seul une distance de trois kilomètres à une vitesse régulière évaluée à $30 \mathrm{~km} / \mathrm{h}$. Andraud demanda d'emblée à élever un moulin à vent pour comprimer l'air, et pour utiliser la force inépuisable et gratuite du vent. Comme toute nouvelle invention, observait L'Illustration ${ }^{161}$, la locomotion à air comprimé rencontra des admirateurs enthousiastes et des détracteurs passionnés. Pour le Journal des Chemins de fer, le but d'Andraud était quand même atteint : «son premier succès a déjà convaincu beaucoup d'incrédules : la locomotive à air peut marcher... » 
81 Au cours des années 1870 et 1880 , le principe de propulsion par l'air comprimé fut adapté avec succès à des tramways urbains, conçus par l'ingénieur Louis Mékarski et exploités à Paris, à Nantes et dans de nombreuses autres villes ${ }^{162}$. Mais, après sa réussite pionnière dans ce domaine, reconnue par Mékarski, Andraud semble avoir abandonné l'air comprimé dans des récipients embarqués pour expérimenter un autre mode de propulsion par l'air comprimé utilisant plutôt des tubes propulseurs flexibles, confectionnés avec de fortes étoffes préparées dans des dissolutions de caoutchouc. Le wagon directeur serait équipé d'un "piston-laminoir ", deux rouleaux verticaux en bronze poli, serrant le tube. De l'air comprimé produit, en principe, par des forces hydrauliques ou des moulins à vent, serait injecté dans le tube qui se gonflerait jusqu'aux deux rouleaux. S'opposant au passage de l'air, ceux-ci prendraient un mouvement de rotation pour pousser tout le convoi en avant. À l'usage des villes, Andraud proposait une version suspendue de ce chemin, portée de distance en distance sur des poteaux. Le tube propulseur serait placé en haut sur une poutre-rail avec les wagons, composés de deux compartiments, suspendus en dessous comme une sorte de double palanquin ${ }^{163}$. Le 10 janvier 1846, Andraud réussit à obtenir une concession pour l'expérimentation de son procédé de locomotion utilisant l'air comprimé dans un tube élastique: une ligne de $4,2 \mathrm{~km}$, destinée aux passagers mais aussi au transport du plâtre, des fruits et des produits maraîchers, devait relier Asnières à Argenteuil. Les souffleries d'air devaient être établies à la gare d'Argenteuil. Cependant, n'étant pas parvenu à réunir les fonds nécessaires, l'inventeur concéda tous ses droits pour cette ligne à la compagnie du Saint-Germain. Mais en avril 1849, Andraud était de retour, cette fois-ci avec un nouveau "chemin éolique ", établi à titre expérimental sur les Champs-Élysées pendant la durée de l'Exposition de l'Industrie. Ce nouveau système, breveté le 6 avril 1846, comportait une espèce de serpent gonflable fixé sur les traverses entre les rails d'une voie ordinaire. Ce serpent était composé de trois parties : un tube intérieur en toile de coton à cinq ou six épaisseurs, enduit de caoutchouc; un fort tube en toile de chanvre, tissée sans couture et pouvant supporter une pression de cinq ou six atmosphères; et, enfin, une toile de recouvrement protectrice. Mis en communication avec un réservoir d'air comprimé, le tube se gonflait, poussant un «cylindre-tracteur » en cuivre attaché horizontalement sous la première voiture d'un convoi. Le poids de la voiture communiquait au cylindre un mouvement de rotation, entraînant le convoi comme s'il était continuellement sur le penchant d'une côte inclinée à 50 degrés. L'Illustration fit des vœux pour que le public, en venant examiner le spécimen de $300 \mathrm{~m}$ de longueur, pût juger par lui-même de l'avenir qui serait réservé au nouveau système du fervent inventeur: "On peut dire de lui que s'il ne vit pas seulement de l'air qu'il respire, au moins il vit tout entier pour lui ${ }^{164}$. »

Les multiples projets d'Andraud peuvent faire ressentir quelque chose de l'effervescence inventive qui régnait autour de systèmes de propulsion pouvant remplacer les locomotives sur les chemins de fer. Le nombre de brevets déposés pour le système atmosphérique est un autre indice de cet engouement général. Dans une analyse rétrospective très complète, publiée au début de l'année 1848, Armengaud aîné passe en revue pas moins de 73 brevets d'invention, de perfectionnement ou d'importation demandés en France, jusqu'en juin 1847, pour les chemins de fer atmosphériques, remarquant lui-même que ce nombre très élevé pouvait donner une idée de l'importance qu'on y attachait ${ }^{165}$ (fig. $\mathbf{n}^{\circ}$ 39). 


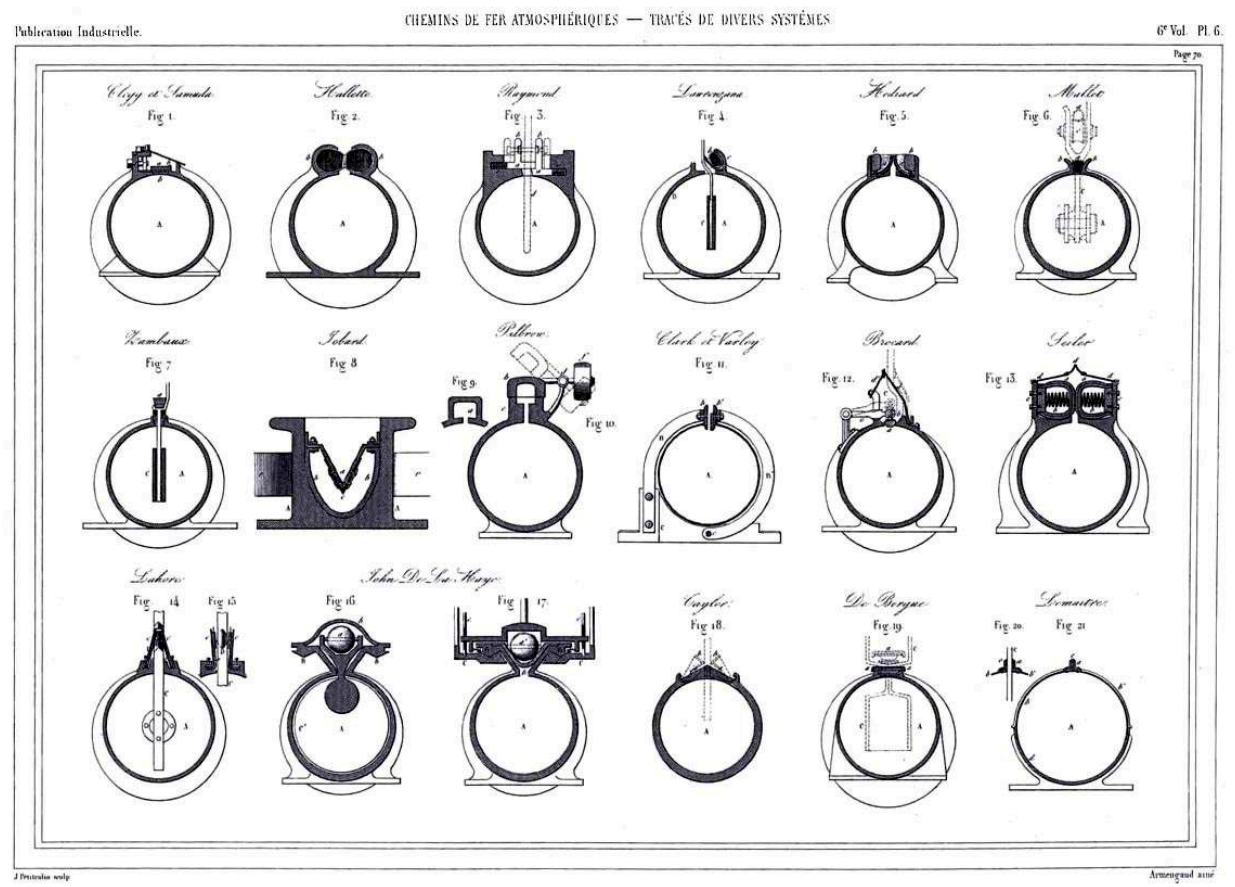

Chemins de fer atmosphériques, tracés de divers systèmes. Armengaud Aîné, Chemins de fer atmosphériques. Publication industrielle des machines, outils et appareils les plus perfectionnés et les plus récents employés dans les différentes branches de l'industrie française et étrangère, 1848. Planche 6.

(c) Société d'Encouragement pour l'Industrie nationale, Paris.

De tels brevets, il faut peut-être le souligner ici, étaient à la portée d'inventeurs n'ayant pas nécessairement une expérience pratique des chemins de fer. "L'horizon scientifique, remarqua L'Illustration, a été un instant obscurci par une nuée effrayante de projets qui n'avaient d'atmosphériques que le nom et dont le silence public a fait bonne justice ${ }^{166}$. $\gg$ Bon nombre de ces projets restaient en effet sur papier au ministère des Travaux publics et de l'Agriculture, où Armengaud les compulsait ${ }^{167}$, mais certains arrivèrent à l'attention de l'Académie des Sciences, à celle de la Société d'Encouragement ou encore à celle du Conseil des Ponts et Chaussées. L'emploi du temps de ce dernier, au cours des années 1844 et 1845, fut considérablement alourdi par leur examen. Quelques-uns semblaient mériter une enquête par des commissions ad hoc, d'autres devant se contenter de simples témoignages d'estime, d'autres encore comme le procédé "mal expliqué et peu intelligible» de M. Geoffroy pour une locomotive à réaction ${ }^{168}$ - étant simplement mis de côté et oubliés.

\section{Pierre Arnollet}

Les " perfectionnements apportés au système atmosphérique des chemins », brevetés le 23 juillet 1844 par Pierre Arnollet, ingénieur en chef des Ponts et Chaussées en retraite ${ }^{169}$, sortaient un peu du lot en proposant une solution à l'un des inconvénients majeurs du système atmosphérique, à savoir l'intermittence de marche des machines fixes, ne faisant un travail utile que pendant une dizaine de minutes par heure mais nécessitant le maintien de la température des chaudières pour que les pompes soient toujours prêtes à fonctionner. Le chaudronnier Louis-Nicolas Lemaître, beau-frère et 
associé de François Cavé, constructeur mécanique bien connu sur la place parisienne, installé dans le faubourg Saint-Denis ${ }^{170}$, faisait des expériences sur ce point dans ses ateliers de La Chapelle-Saint-Denis, utilisant un ventilateur pour activer ponctuellement la combustion sous une chaudière de sa fabrication. Quant à Arnollet, son invention consistait dans l'interposition, entre la machine fixe et le tube de propulsion, de réservoirs "de vide». Ceux-ci auraient une capacité égale à trois fois celle du tube et l'air en serait épuisé par des machines à vapeur fonctionnant de manière continue. À la différence des machines très puissantes requises par le système de Clegg et Samuda, exerçant leur action directement sur le tube, les machines du système Arnollet pouvaient être de puissance bien plus faible - de dix chevaux seulement -, d'où d'importantes économies de combustible. Les réservoirs, selon l'inventeur, pouvaient être élevés en maçonnerie de pierre meulière et ciment romain avec une voûte de ciment romain, le tout enduit de goudron. Encore mieux, ils seraient « de profondes caves accolées, construites à chaux hydraulique, avec précaution, et revêtues extérieurement de ciment romain et de bitume ou de goudron ${ }^{171}$ ». Dans son rapport sur ce système, Gabriel Lamé formula de sérieuses réserves quant à la solidité et l'imperméabilité de tels réservoirs, devant résister à une pression atmosphérique qu'il estimait à environ sept tonnes par mètre carré. Les dépenses de construction d'une structure d'un genre si nouveau seraient-elles moindres, en fin de compte, que celles des grosses machines et pompes à air exigées par le système atmosphérique à l'anglaise?

Figure 40

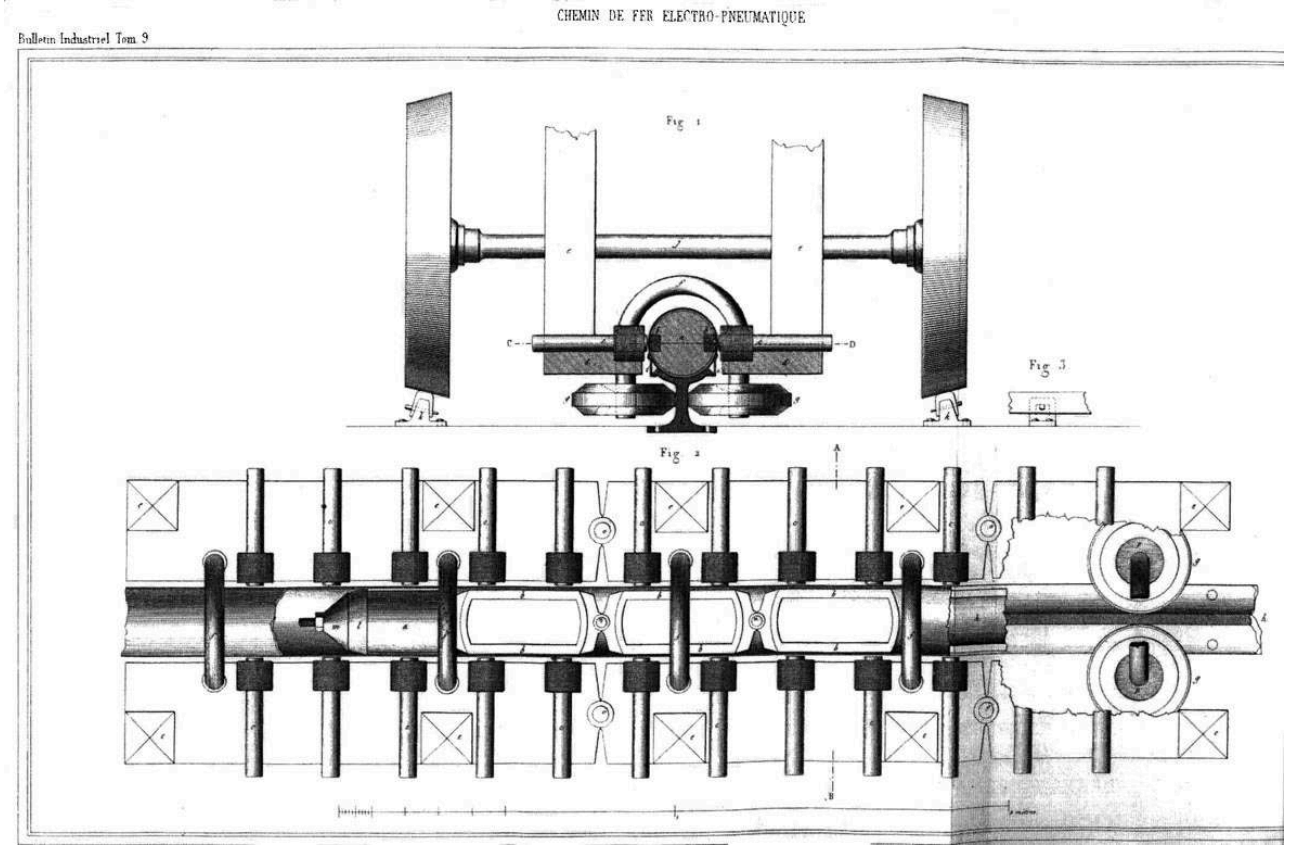

Chemin de fer électro-pneumatique proposé par M. Jobard. BULLETIN INDUSTRIEL, tome 9.

(c) Institution of Civil Engineers.

La plupart des inventeurs, cependant, cherchaient un remède à l'autre faiblesse majeure du système de Clegg et Samuda, les fuites d'air par la soupape longitudinale. Parmi ces inventions, deux peuvent retenir ici notre attention pour avoir donné lieu à des essais pratiques en grandeur réelle et suscité de grands espoirs, teintés de fierté 
nationale. Mentionnons d'abord, toutefois, et plutôt pour son intérêt littéraire, le brevet pris par Jean-Baptiste Jobard, de Bruxelles, le 30 juin 1846, pour un «chemin de fer électro-pneumatique dont le principe peut aussi recevoir d'autres applications » (fig. $\mathbf{n}^{\circ} \mathbf{4 0}$ ). Convaincu, comme tant d'observateurs anglais et français, que les locomotives représentaient «le chancre des chemins de fer ", mais conscient aussi des faiblesses inhérentes à la soupape de Clegg et Samuda, Jobard reprit et développa une idée sommairement énoncée dans le brevet Pinkus de $1834^{172}$, celle d'un tube de propulsion en cuivre, en zinc ou en tôle de fer de quelques millimètres d'épaisseur sans aucune ouverture longitudinale. Propulsé par air comprimé, préféré par Jobard à la pression atmosphérique, le piston dans le tube serait garni d'électro-aimants pouvant entraîner, par un lien "immatériel ", les électro-aimants attachés sous le wagon directeur ${ }^{173}$. Rien n'indique qu'un tel procédé, sur lequel travaillaient apparemment d'autres inventeurs en Italie, en Suisse et à Mulhouse, ait eu une quelconque application $^{174}$ mais elle capta l'attention du jeune Jules Verne. Dans son roman d'anticipation Paris au XX ${ }^{e}$ siècle, rédigé vers 1863 et dont l'action se déroule en 1960, le chemin de fer métropolitain de la capitale établi, selon Verne, depuis 1913, utilise le système préconisé par le célèbre ingénieur belge « qui florissait vers le milieu du dixneuvième siècle ${ }^{175}$ ». L'air comprimé du roman est fourni par la Société des Catacombes de Paris et de la force motrice à domicile, société disposant de centaines de moulins à vent dans la plaine de Montrouge et stockant dans les souterrains de la capitale l'air comprimé par ces moulins...

\section{Hédiard}

Figure 41

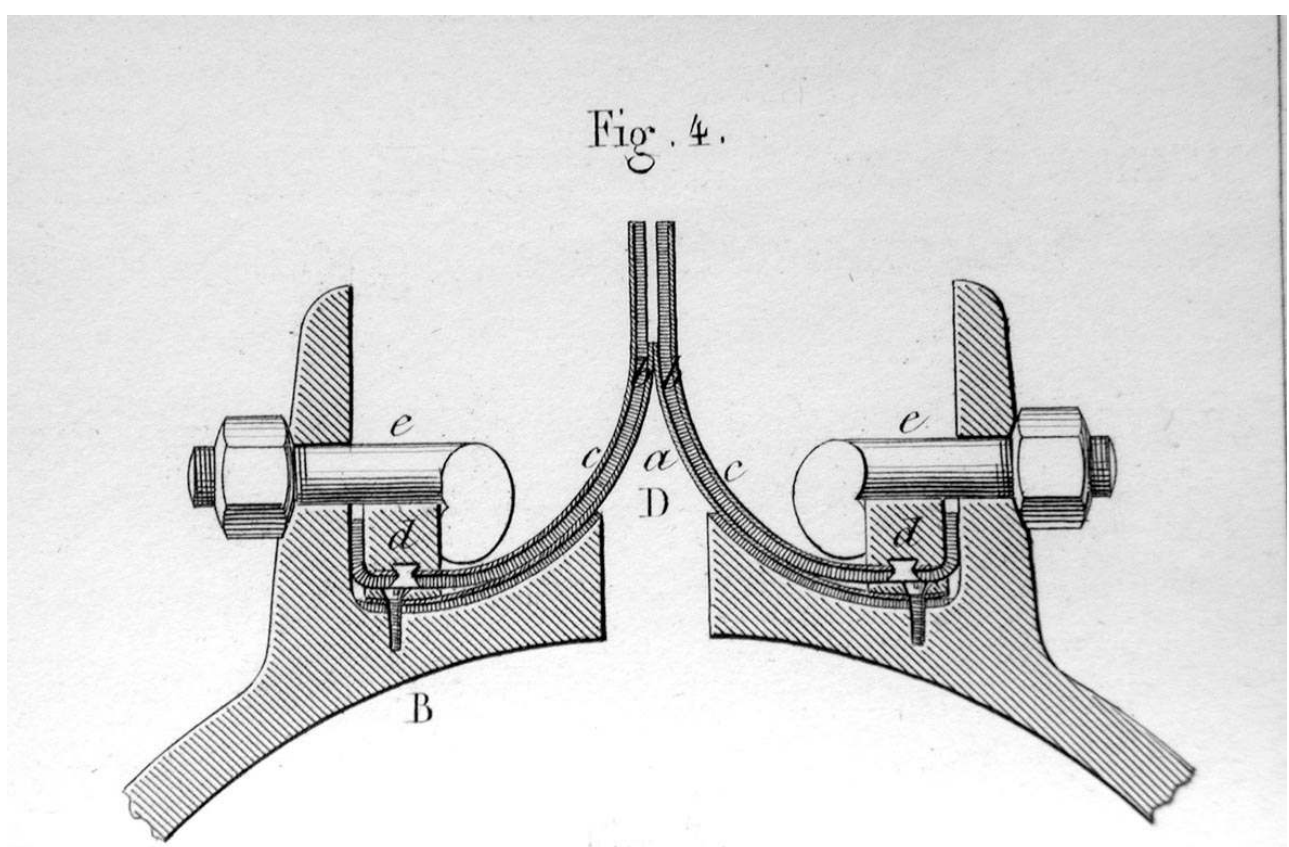

Coupe transversale sur la soupape aux lèvres d'acier d'Hédiard. BULLETIN DE LA SOCIÉTÉ D'enCOURAgement POUR L'INDUSTRIE NATIONALE, 1848, planche 1078.

(C) Société d'Encouragement pour l'Industrie nationale, Paris. 
Le 27 novembre 1844, sous le titre de « Tube fermant hermétiquement, applicable au système atmosphérique des chemins de fer ", Hédiard, qualifié d'« industriel », prit un brevet pour un nouveau système de fermeture par une soupape longitudinale en ressorts d'acier, système également esquissé par Pinkus dans un de ses brevets, en 1836. Dans ce système, la rainure pratiquée dans le tube était fermée par deux lames d'acier d'une très faible épaisseur et d'une largeur de onze à douze centimètres. Ces lames étaient recourbées en arc de cercle et fixées au tube de propulsion au moyen de vis taraudées dans la fonte et de tringles pressant sur toute leur longueur. Ainsi s'appuyaient-elles l'une contre l'autre sur une faible surface de leur partie supérieure, le ressort du métal assurant, en théorie du moins, un joint étanche (fig. $\mathbf{n}^{\circ} \mathbf{4 1}$ ). Faite de fortes feuilles de tôle percées de trous et réunies en biseau à leurs extrémités, la « tige directrice ", reliant le piston dans le tube au wagon directeur, fraierait elle-même son passage entre ces lames, en les faisant bâiller légèrement. Pour montrer sa simplicité d'exécution, Hédiard fit fabriquer un spécimen de sa soupape dans les ateliers de Bernard, canonnier, avenue de la Motte-Piquet à Paris (fig. $n^{\circ} 42, n^{\circ} 43$ ), puis réussit à convaincre quelques capitalistes d'investir dans un chemin d'essai pour faire des expériences sur une grande échelle et pour apprécier les avantages de sa soupape par rapport à celle de Clegg et Samuda. Parmi ces investisseurs figurait un certain H. A. Dubern, membre du conseil de surveillance de la compagnie. À la suite d'une visite en Angleterre à la recherche des derniers documents officiels sur la question, Dubern publia en août 1846 un résumé des opinions des ingénieurs français et anglais sur les chemins de fer atmosphériques. Tout en faisant une place aux avis hostiles de Stephenson et de Locke, cette brochure en offre bien davantage à ceux des experts favorables - Cubitt, Brunel, Vignoles, Samuda, Field, Gibbons, Teisserenc, Mallet, Arago - et termine, sans surprise, en vantant les lames d'acier de M. Hédiard ${ }^{176}$. 


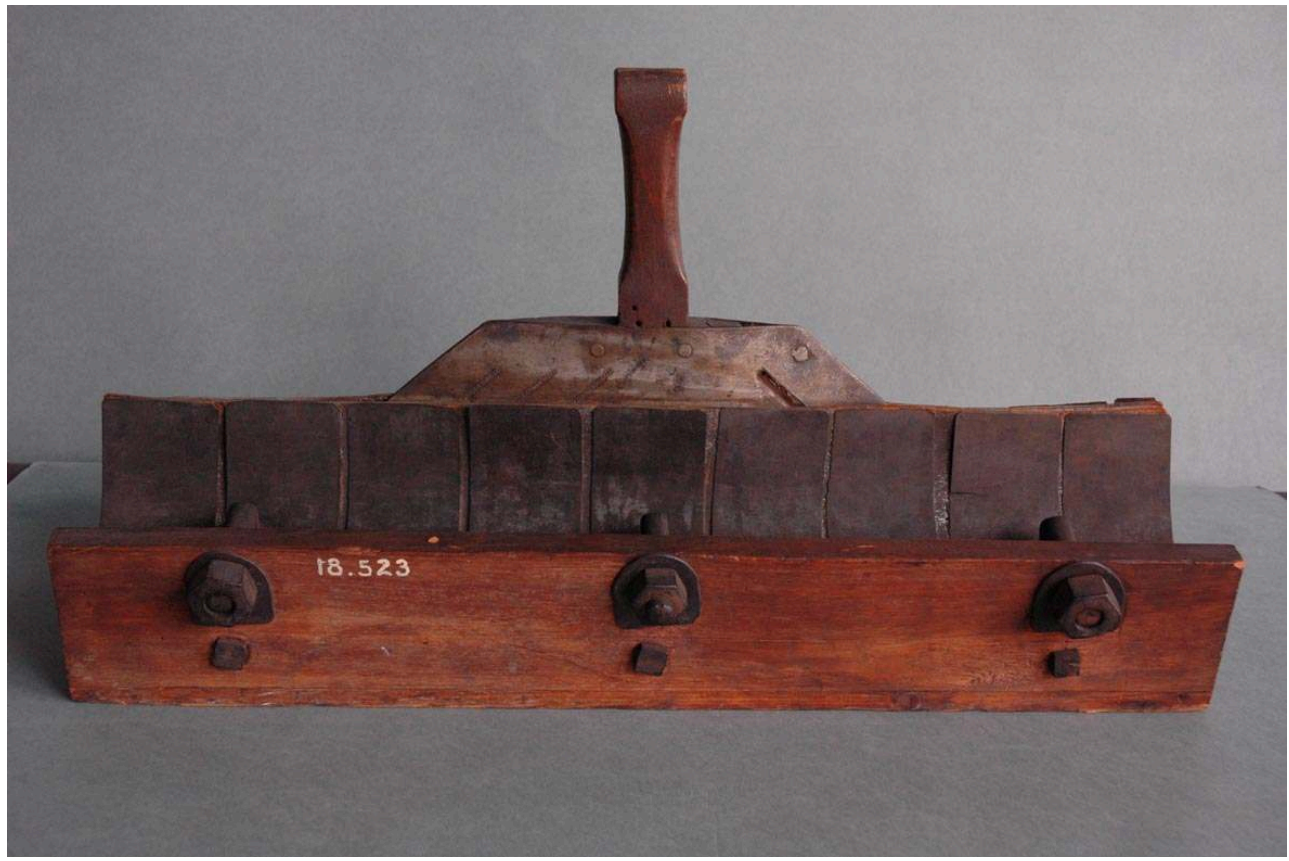

Vue d'un élément de soupape du chemin de fer atmosphérique de Saint-Ouen, entré dans les collections de CNAM en 1907. II s'agit peut-être de l'échantillon fabriqué pour Hédiard en 1844, ne montrant que le principe de la soupape, sans le tube de propulsion. CNAM, Inventaire 18523, réserves de Saint-Denis.

(C) Musée des arts et métiers.

Figure 43

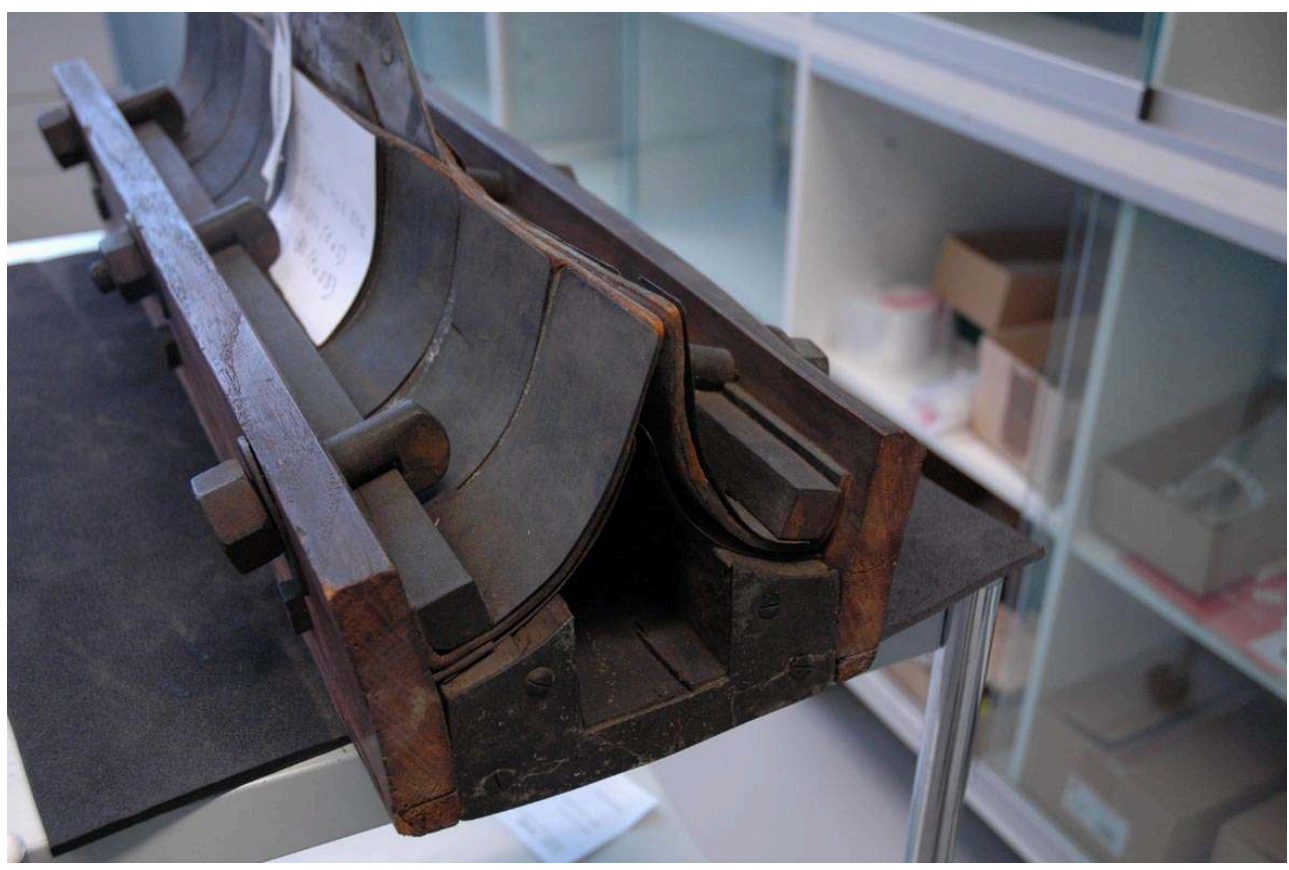

Autre vue d'un élément de soupape du chemin de fer atmosphérique de Saint-Ouen.

(c) Musée des arts et métiers.

Constituée avec l'appui de la banque Ardoin ${ }^{177}$, la Compagnie du Chemin de fer d'essai de Saint-Ouen, placée sous la direction d'un ancien magistrat, Bernède, fit appel à Émile 
Vuigner, déjà connu par son étude de la ligne irlandaise et considéré comme étant capable de diriger les travaux d'établissement du chemin et de son nouveau système de propulsion. Grâce à Jules Ardoin, entrepreneur du port et de la gare d'eau de SaintOuen, un chemin d'essai en boucle, d'une longueur totale de $1697 \mathrm{~m}$ et comportant un tube de propulsion de $595 \mathrm{~m}$ de long, put être établi sur un terrain dépendant de cette gare $^{178}$ (fig. $\mathbf{n}^{\circ} \mathbf{4 4}$ ).

Figure 44

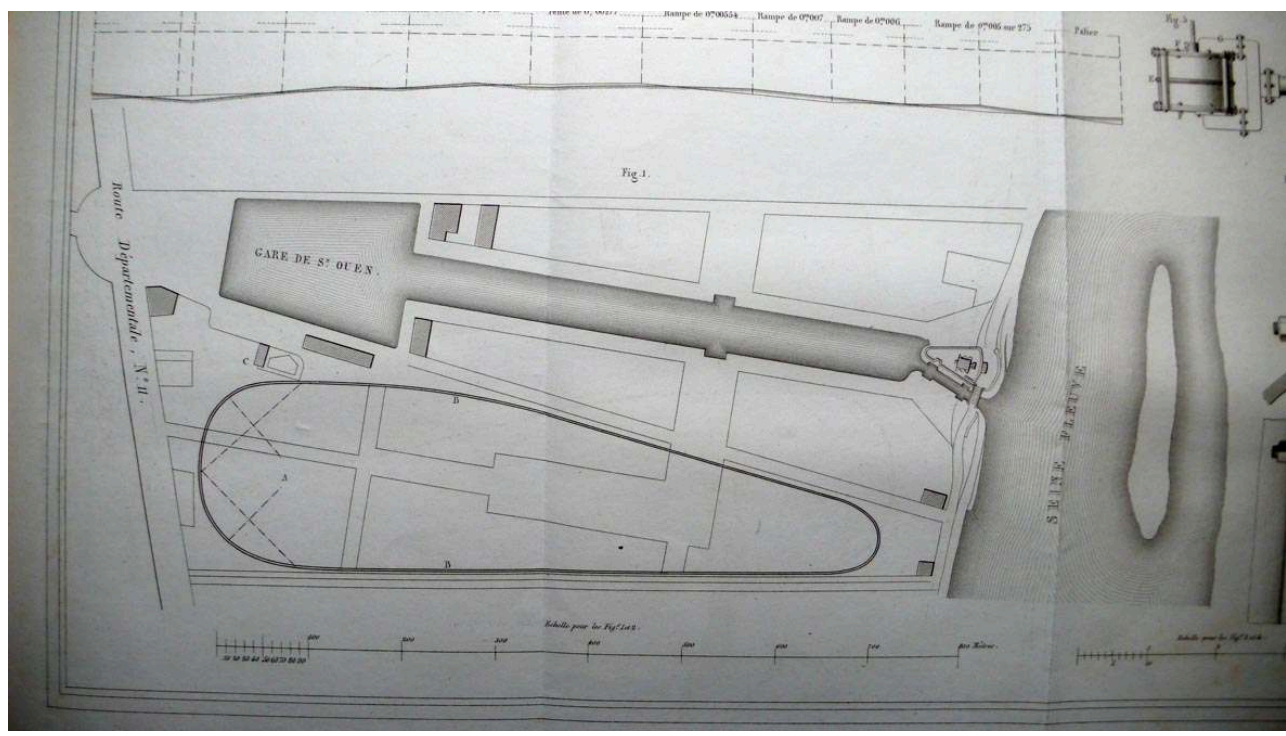

Chemin de fer d'essai établi à Saint-Ouen. A : Polygone fermé de rails. B : Tube propulseur. C : Machine à vapeur. BULLETIN DE LA SOciéTÉ d'ENCOURAgEMENT POUR L'INDUSTRIE NATIONALE, 1848, planche 1078.

(c) Société d'Encouragement pour l'Industrie nationale, Paris.

Le tube, en fonte de deuxième fusion, fut coulé par longueurs de 2,85 m par la maison parisienne de Calla ${ }^{179}$. Par son diamètre intérieur de $40 \mathrm{~cm}$, il était comparable à celui de Dalkey, portant les mêmes nervures de renforcement en forme de croissant, garnies de pattes pour les fixer sur les traverses de bois. Mais à la différence du tube de Dalkey, celui de Saint-Ouen fut fondu ouvert, c'est-à-dire en formant lors de la coulée la rainure longitudinale de trois centimètres de largeur. Comme pour le tube de Dalkey, enfin, l'intérieur du tube ne fut pas allésé, opération trop coûteuse, mais simplement raboté puis enduit d'un mélange de suif et de stéarine. Joly d'Argenteuil, connu en tant que "serrurier", c'est-à-dire constructeur métallique - il participait dans ces mêmes années à la construction des halles de la gare Saint-Lazare -, contribua au chantier en fournissant un chariot roulant pour transporter les tubes, préparés par Calendeau, entrepreneur de fontainerie à Passy. Ce tube fut établi en partie sur palier horizontal et en partie sur une rampe avec une déclivité de six millimètres par mètre. La machine à vapeur à cylindre oscillant, de 40 chevaux, louée à François Cavé, faisait fonctionner une pompe à double effet. Pour le matériel roulant de ce chemin d'essai, étant donné son tracé aux courbes à rayons très courts $(500,80$ et $40 \mathrm{~m})$, imposé par les limites du terrain, Vuigner, conformément par ailleurs à sa volonté de combiner le principe atmosphérique avec celui des trains articulés, put utiliser trois voitures mises à sa disposition par Claude Arnoux, avec l'accord de la compagnie du chemin de fer de Sceaux, et provenant du chemin d'expérience à Saint-Mandé. Le premier wagon, 
portant les galets directeurs du système Arnoux, fut adapté pour porter la tige du piston. Ainsi équipé, le chemin fut expérimenté au cours du printemps de l'année $1846^{180}$. En interposant des bandes de feutre entre les lames et la fonte du tube, et en munissant celles-ci de baguettes horizontales remplies de graisse végétale, le système donna quelques résultats encourageants. Sur la longueur du tube de propulsion, une vitesse moyenne de $36 \mathrm{~km}$ à l'heure fut atteinte, et, en poussant le vide, une vitesse de $72 \mathrm{~km}$ à l'heure fut même enregistrée. La tige ne s'échauffait pas de manière sensible, et les ressorts formant la soupape reprenaient sans altération leur position première. Mais, malgré le soin apporté à la fabrication et à la pose de ceux-ci, des rentrées d'air considérables eurent lieu lorsque la graisse ne couvrait pas leur partie supérieure. Quand les ressorts étaient en revanche bien lubrifiés, la graisse s'introduisait dans le tube lors du passage de la tige directrice, et, chassée par le piston, venait jaillir en masse au bout du tube. Par temps ensoleillé, la dilatation de celui-ci compromettait l'herméticité des joints entre les sections, faits d'abord avec de la corde bitord imprégnée de goudron, ensuite avec de la glu marine. Le 7 novembre 1847, au nom du comité des arts mécaniques de la Société d'Encouragement, Louis-Alphonse Baude conduisit quelques expériences pour évaluer l'efficacité de la soupape Hédiard, mais, tout en louant la persévérance et le dévouement avec lesquels les travaux avaient été menés, son rapport à la Société, publié en octobre 1848, constata que le chemin d'essai n'existait plus ${ }^{181}$

\section{Alexis Hallette}

\section{Figure 45}

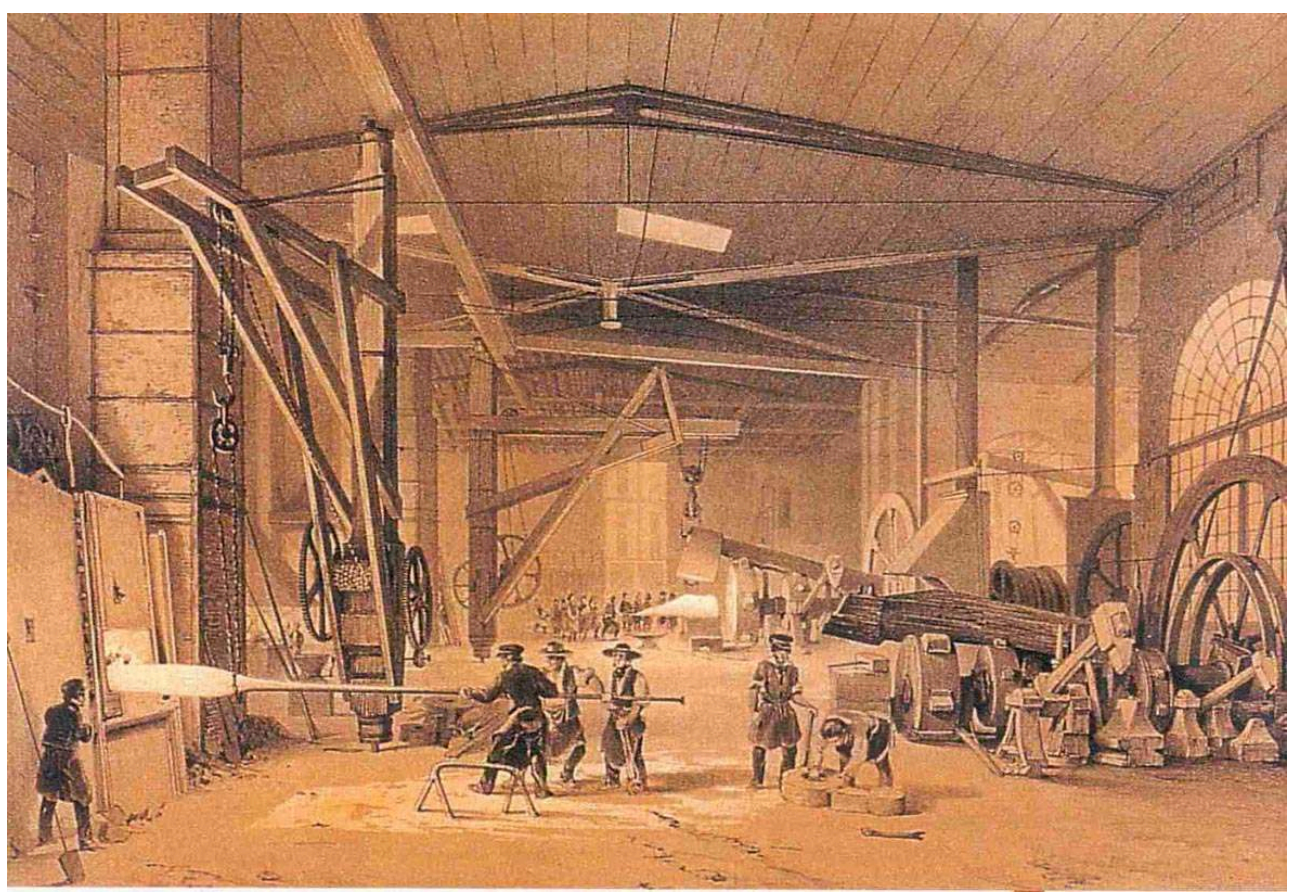

Ateliers de construction de M. Hallette à Arras, Grandes Forges, 1847, lithographie de Nicolas Chapuy d'après François Bonhommé.

(c) BnF 
Bien plus que ces «lèvres d'acier » d'Hédiard, ce sont les «lèvres pneumatiques » d'Alexis Hallette qui concentrèrent sur elles, à cette époque, les meilleurs espoirs d'une solution française aux problèmes d'étanchéité de la soupape longitudinale. Alexis Hallette, selon François Caron, était l'un des mécaniciens les plus brillants de son temps ${ }^{182}$. Né à Lille en 1788 , fils d'un fabricant de lacets, il fit, en 1806, un court passage dans les ateliers de peinture de la manufacture de Sèvres avant de créer, en 1813, à Arras, un atelier de construction mécanique. Équipant l'industrie naissante du sucre de betterave, construisant des moulins à vent puis de grandes machines à vapeur fixes et de navigation maritime et fluviale, ainsi que des mécaniques pour les filatures de Lille et de Roubaix, les « Fonderie et ateliers de construction de machines à vapeur et autres, forges de grosses œuvres et fabrication de fer de mitrailles " sont connus par la description qu'en fit Calla en $1842^{183}$ et par une monographie, dans la série "Grands établissements industriels de la France", publiée par L'Illustration ${ }^{184}$ (fig. $\mathbf{n}^{\circ}{ }^{45}$ ). L'entreprise était devenue dans les années 1840 l'une des principales usines de construction mécanique en France, s'étendant sur plus de deux hectares et faisant travailler jusqu'à six cents ouvriers. En janvier 1827, Hallette avait accompagné Marc et Charles Seguin en Angleterre pour l'achat de deux locomotives à vapeur chez les Stephenson à Newcastle, une de ces locomotives étant livrée à Arras ${ }^{185}$. Ces liens ferroviaires avec les Seguin n'eurent pas de suite mais, en 1842, les ateliers d'Arras furent réorganisés en vue de la construction de locomotives, une douzaine par an, commandées cette fois-ci pour la Compagnie du Nord. Dès cette époque, toutefois, et peut-être avant, Hallette s'était intéressé à la propulsion atmosphérique et pensait avoir trouvé la parade aux fuites d'air de la soupape de Clegg et Samuda. Le 5 février 1844, il fit présenter par François Arago, à l'Académie des Sciences, un modèle réduit destiné à faire comprendre son nouveau moyen de fermeture de la soupape. De part et d'autre de la fente, deux gouttières, faisant corps avec le tube et se regardant par leur concavité, logeaient chacune un boyau en tissu souple et parfaitement étanche, pour l'air comme pour l'eau. Gonflés à l'air comprimé, ces boyaux se pressaient l'un contre l'autre, empêchant l'air extérieur de rentrer dans le tube (fig. $\mathbf{n}^{\circ}{ }^{46)}$. La tige reliant le piston aux wagons avait une section horizontale en forme de lentille pour mieux glisser entre les boyaux qui étaient renforcés de manchons de cuir à leur point de contact (fig. $\mathbf{n}^{\circ}$ 47). Dans un article publié dans Le Progrès du Pas-de-Calais le 10 juillet 1846, Frédéric Degeorge, directeur de ce journal et ami de l'industriel, fit un récit assez romanesque des circonstances de cette invention: Hallette, assis dans son cabinet, médite sur le système à clapets de Clegg et Samuda, plongé dans une rêverie, un crayon à la bouche... "sa main promène machinalement son crayon sur ses lèvres, un moment d'immobilité survient, l'idée, la grande idée a jaillii ${ }^{186}$ !» 
Figure 46

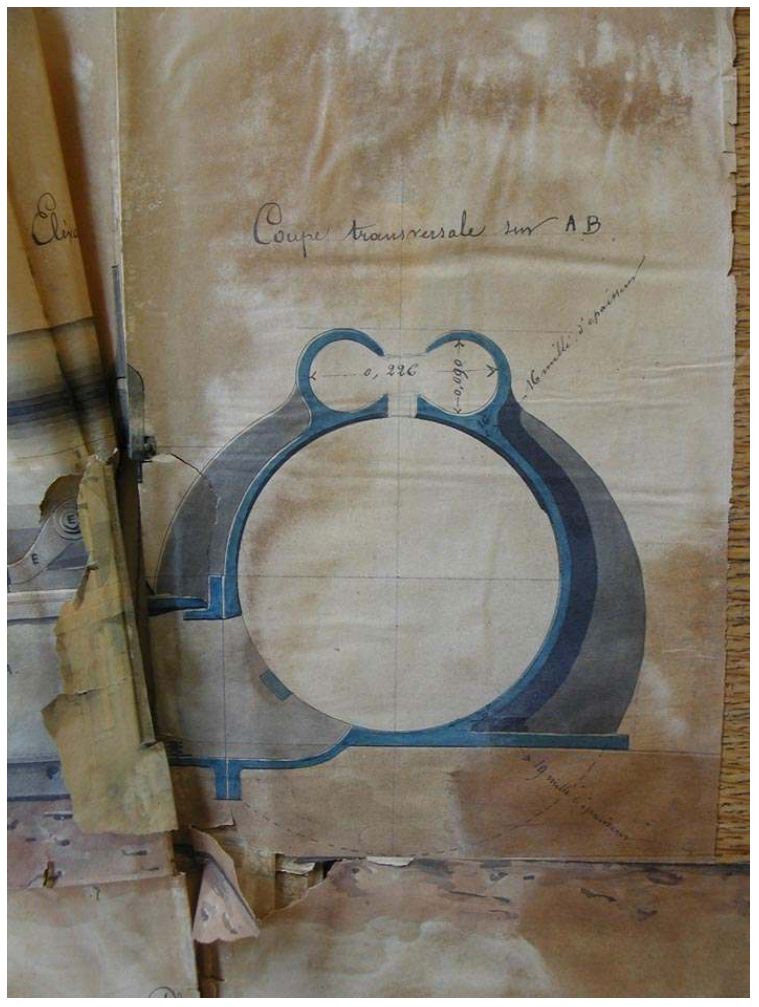

Coupe transversale sur le tube de propulsion de Hallette, montrant gouttières destinées à contenir les boyaux gonflés à l'air comprimé. Ce dessin, et les quatre suivants, sont vraisemblablement de la main de Hallette lui-même. Archives nationales F/14 11171.

(c) Archives nationales. 


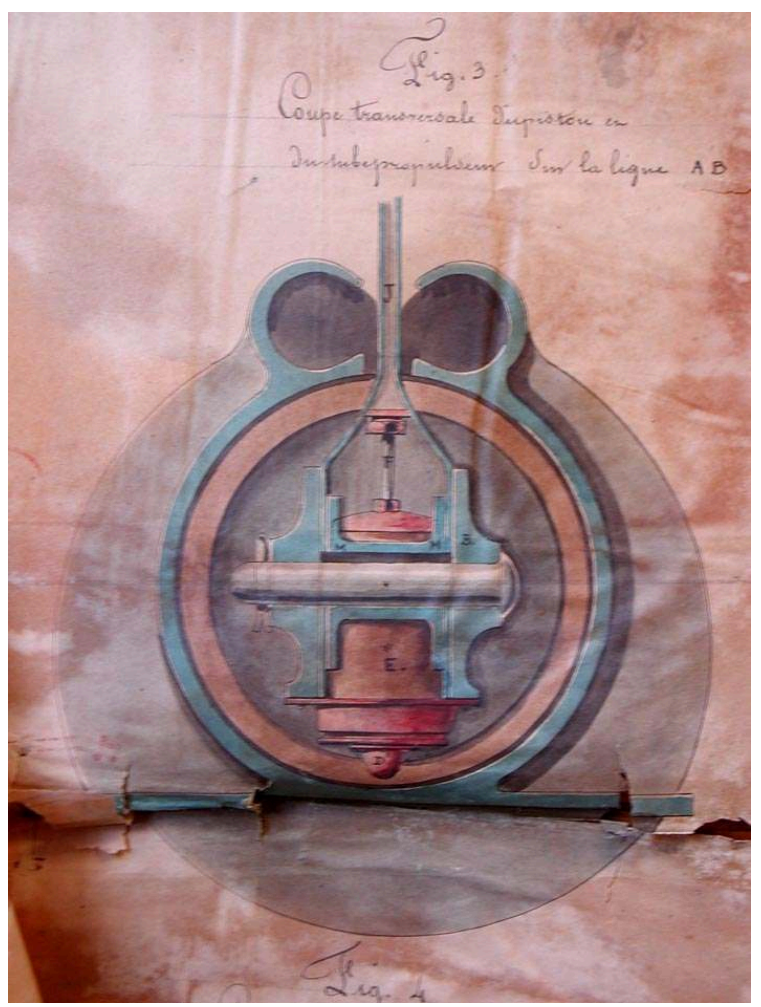

Coupe transversale du tube de propulsion au moment du passage du piston, la tige de connexion $\mathrm{J}$ passant entre les lèvres pneumatiques. Archives nationales F/14 11171.

\section{(c) Archives nationales.}

Sous le titre de "système de tube propulseur à fermeture hermétique ", l'invention fut brevetée le 6 mars 1844, quelques semaines après la diffusion par l'inventeur d'une première note sur son nouveau tube propulseur ${ }^{187}$. Hallette, bien évidemment, est convaincu de la supériorité de son système, qu'il dit avoir mûri depuis huit ans. Ce qui le caractérise, c'est la simplicité, comparée à la caractéristique générale de l'invention de Clegg et Samuda, sa complication. Au lieu d'être mécanique, cette simplicité empruntait à la nature : « les lèvres, humides, élastiques, susceptibles de compression partielle, permettent entre elles le jeu d'une tige de fleur, d'un crayon, sans que le mouvement laisse introduire de l'air dans la bouche. C'était le modèle à suivre, il fallait faire des lèvres à la bouche longitudinale du tube [...] Les boyaux agissent alors absolument comme les lèvres; ce sont des lèvres artificielles, mais véritables, qui permettent sans difficulté et presque sans frottement le jeu le plus rapide du bras communicateur du piston, sans que l'air puisse en profiter pour entrer dans le tube propulseur [...] Il y a ni galets, ni roulettes, ni soupapes ferrées, ni boulons, ni composition fusible, ni réchaud pour la fondre [...] Ceux qui croient à l'efficacité des moyens anglais ne pourront s'empêcher de reconnaître la supériorité de notre système, dont nous sollicitons vivement l'examen et la première application en France, pour que le nom que nous lui avons donné de système français ne puisse lui être disputé sous aucun rapport. » 
Figure 48

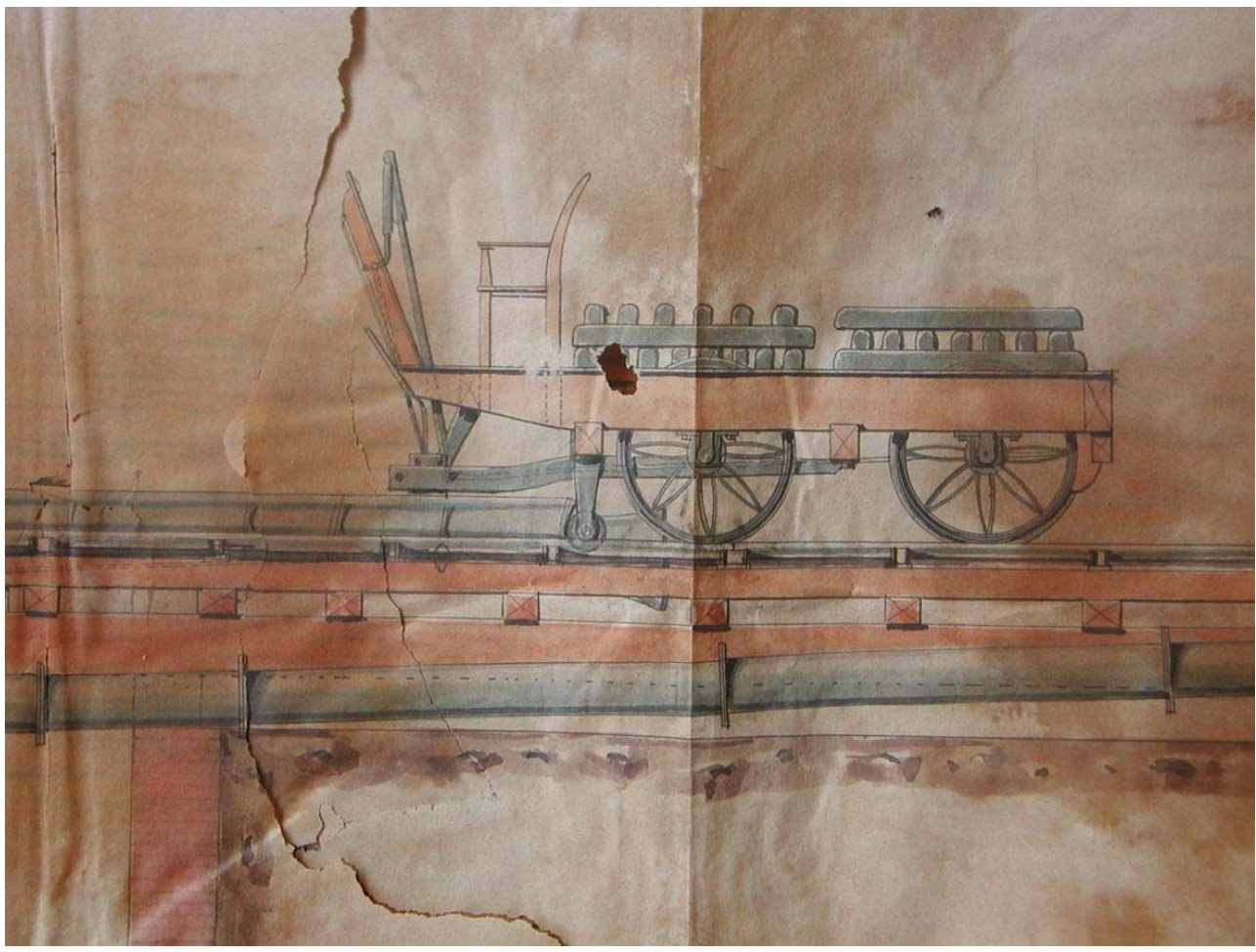

Wagon directeur du chemin de fer de Hallette à Arras, la tige de connexion entrant dans le tube. Archives nationales F/14 11171.

(c) Archives nationales. 


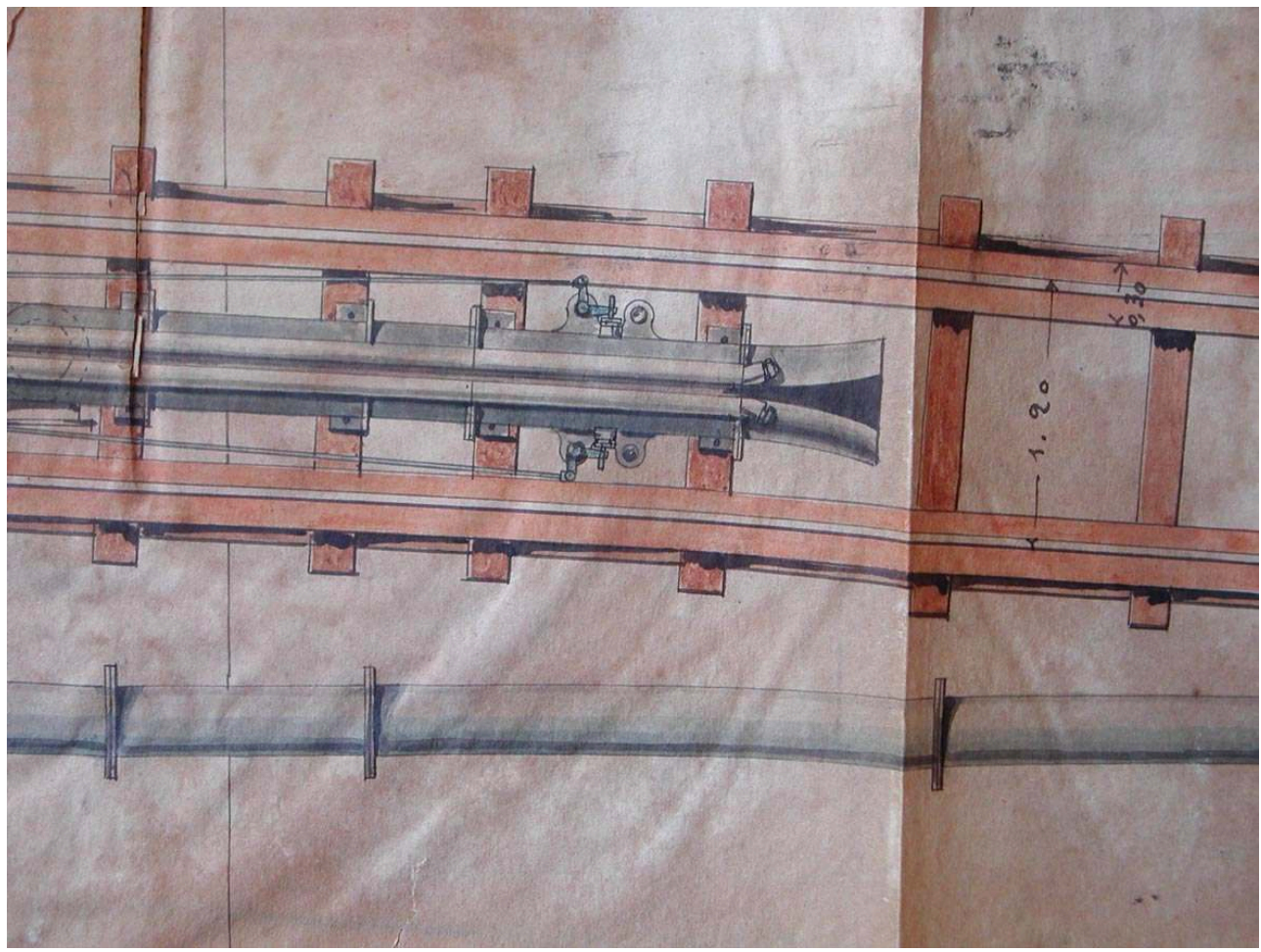

Plan d'une partie du chemin de fer de Hallette à Arras, montrant l'entrée du tube de propulsion. Archives nationales F/14 11171.

(c) Archives nationales.

Dans ses ateliers d'Arras, sous un hangar près de l'une des quatre machines à vapeur de l'usine, Hallette se dépêcha d'établir un spécimen en grandeur réelle de son système " français » de chemin de fer atmosphérique, y investissant $30000 \mathrm{~F}$ au total. Long de $122,43 \mathrm{~m}$, ce chemin de fer était équipé d'un tube de propulsion de $26,85 \mathrm{~m}$ de long et de $38 \mathrm{~cm}$ de diamètre intérieur, le "standard » de Dalkey. Les gouttières servant à loger les boyaux gonflés d'air avaient un diamètre intérieur de $9 \mathrm{~cm}$. Après un passage, sans tube, à travers une rue qui séparait les ateliers, un deuxième tube, de $17 \mathrm{~m}$ de long, sans soupape, était destiné à simuler l'interruption du tube dans les gares d'évitement. Avec un wagon pesant $5410 \mathrm{~kg}$, non compris les hommes qui le montaient (fig. $\mathbf{n}^{\circ} \mathbf{4 8}$ ), ce chemin de fer fit une première démonstration de l'efficacité "parfaite» de son joint hermétique en juin 1844, devant une commission nommée par l'Académie d'Arras ${ }^{188}$ (fig. $n^{\circ}$ 49). Par la suite, des commissions parisiennes défilèrent dans les ateliers d'Arras, où le public était également admis le dimanche, entre midi et $14 \mathrm{~h}$. Au nom de la commission nommée par l'Académie des Sciences, le baron Séguier rendit compte, dans la séance du 9 mars 1846, de sa visite à Arras. Quoique grossièrement exécuté, « l'organe principal du système, le mode de fermeture, ne laisse, dès à présent, rien à désirer au point de vue de l'occlusion. » Grâce à des lèvres pneumatiques gonflées un peu plus fortement que sur le tube horizontal, un tube placé verticalement pouvait être rempli d'eau sans qu'aucune fuite ne se produise. Composée de T. Olivier, J. F. Saulnier et F. C. Calla, la commission envoyée par le comité des arts mécaniques de la Société d'Encouragement pour l'Industrie nationale rendit son rapport en mai $1846^{189}$, donnant au système de fermeture de $\mathrm{M}$. Hallette son "approbation tout entière, comme résolvant d'une manière satisfaisante le problème théorique, savoir, maintenir le vide 
dans un long tuyau. » Mais le rapporteur, Théodore Olivier, ne voulait pas se prononcer sur la valeur industrielle de cette solution, en attendant, pour formuler ses doutes à cet égard, qu'une exploitation réelle et suivie pendant deux ou trois ans permette de juger en connaissance de cause.

Pour le Conseil des Ponts et Chaussées, une commission composée de Kermaingant, inspecteur général, Le Masson, inspecteur divisionnaire-adjoint, Bélanger, ingénieur en chef, et Mallet, inspecteur divisionnaire, séjourna à Arras du 23 au 26 décembre 1844. Rédigé par Mallet, décidément l'expert en matière atmosphérique, le rapport de cette commission fut rendu le 5 avril $1845^{190}$. Il était également favorable à la soupape Hallette : "Nous la regardons comme supérieure, pour la conservation du vide, à la soupape anglaise [...] M. Hallette a trouvé le moyen de paralyser une partie des rentrées d'air dans les chemins de fer atmosphériques. » La preuve ? Lorsque la pompe faisait le vide dans le tube, «une flamme de chandelle promenée le long de la fente restait verticale. Elle n'était pas non plus attirée vers les bords de l'enveloppe en fonte. » Mais Mallet ne dissimulait pas non plus les accidents qui pouvaient arriver aux lèvres: «Pendant que la machine pneumatique continuait d'agir, nous avons été surpris par un bruit semblable à une détonation. C'est que les lèvres sur une certaine partie de leur longueur étaient rentrées dans le tube: qu'elles avaient été, selon l'expression des ouvriers, avalées. Pour que cet effet se produise, il faut qu'elles passent à travers l'ouverture de $4,5 \mathrm{~cm}$ qui forme la rainure longitudinale. " La résistance du boyau gonflable, était, en effet, problématique, et Hallette se plaignait de ne pas trouver de tisserand capable de confectionner des boyaux courbes ${ }^{191}$. Ce boyau était fabriqué d'une étoffe composée de plusieurs tissus superposés, comprenant entre eux une couche de caoutchouc à laquelle ils adhéraient fortement. Extérieurement et intérieurement, il était ensuite couvert d'un autre enduit de caoutchouc. Hallette utilisait à Arras un tissu fabriqué selon un procédé de M. Wetzlar, de Leipzig, mais regardait aussi outre-Manche où un $\mathrm{M}$. Brockedon, de Londres, commençait à fabriquer un caoutchouc imperméable, conservant en toute saison son élasticité et son inaltérabilité à l'huile, par un nouveau procédé appelé "vulcanisé ${ }^{192}$. Hallette hésitait aussi sur la meilleure manière d'attacher aux boyaux les bandes de cuir protectrices : par un lacet très mince, mais qui pourrait créer des interstices facilitant des entrées d'air? Ou par collage, plus lisse, mais rendant plus difficile le renouvellement des cuirs? Il cherchait surtout à améliorer le fonctionnement de son piston, qui mesurait deux mètres de long, comparé à six mètres pour le châssis de piston Clegg et Samuda. La tige de connexion du piston Hallette était verticale, et ne devait plus jouer un rôle d'ouverture de la soupape, l'appel d'air atmosphérique s'effectuant plutôt au moyen de soupapes spéciales placées sur le côté latéral du tube propulseur à 500 ou $1000 \mathrm{~m}$ les unes des autres et ouvertes par l'effet de galets attachés au wagon-directeur. La tige devenait dès lors plus mince, de deux centimètres d'épaisseur au lieu de 3,8. Elle devait servir néanmoins de passage à quatre tubes, l'un pour la communication du baromètre placé dans la voiture directrice, un autre pour le système de soupape donnant passage à l'air atmosphérique devant le piston, un autre amenant l'huile destinée à prévenir son échauffement et à lubrifier les lèvres, et un quatrième, enfin, attaché à une petite pompe à air également placée dans la voiture directrice. Cette dernière servait à enfler une garniture pneumatique autour du piston, un coussin annulaire retenu entre deux coquilles. Protégé par un cuir graissé, ce coussin en légère saillie devait assurer une meilleure étanchéité entre le piston et la paroi intérieure du tube ${ }^{193}$ (fig. $\mathbf{n}^{\circ}$ 50). Ce dernier dispositif séduisit William Cubitt : « Le célèbre ingénieur anglais a visité dernièrement 
le chemin d'essai d'Arras et la disposition de cette garniture de piston lui a semblé devoir donner des résultats tellement supérieurs sur celle employée habituellement qu'il n'a pas hésité à donner à M. Hallette la commande d'un piston complet sur les dimensions qu'il lui enverrait ${ }^{194}$."

Figure 50

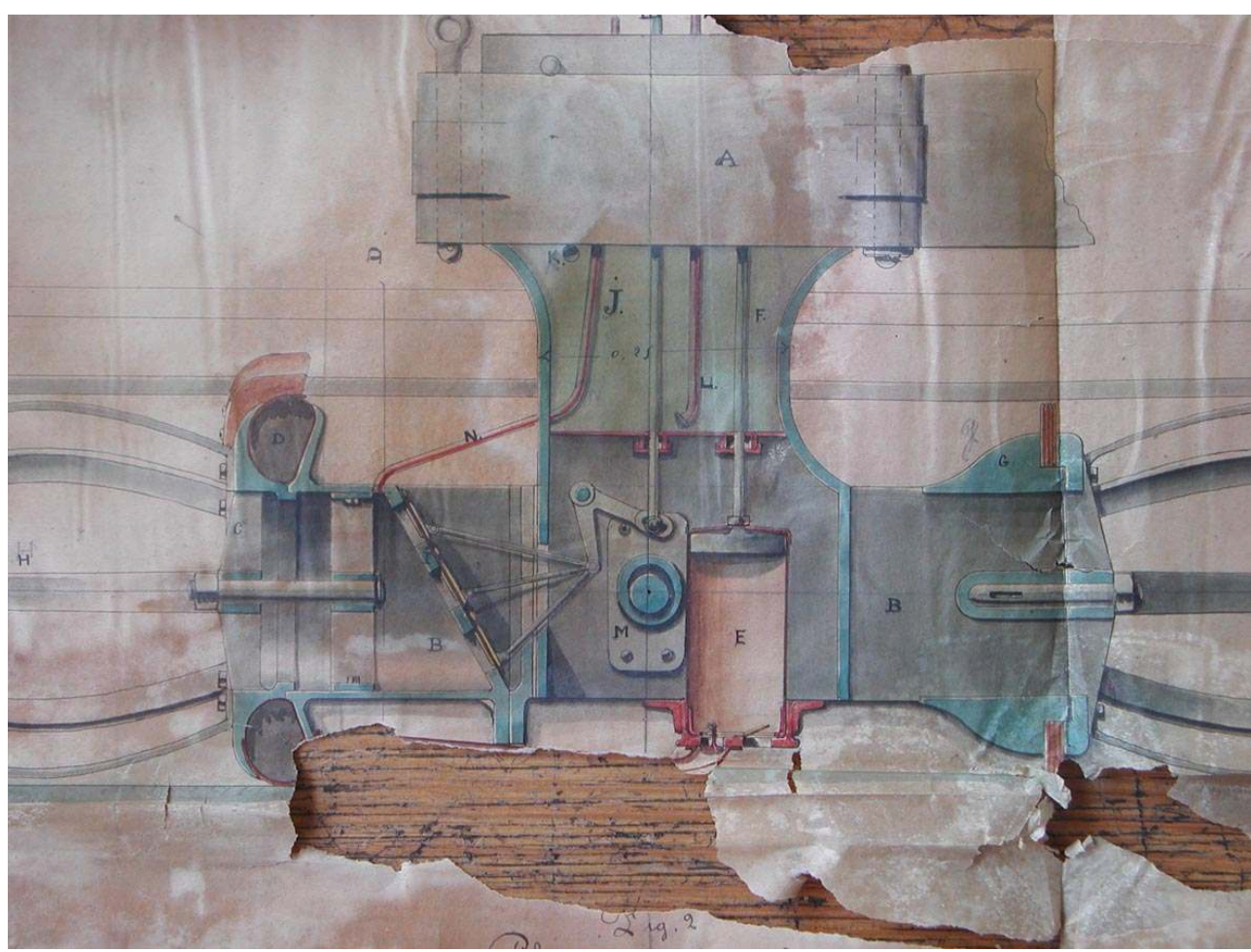

Coupe longitudinale de la partie centrale du piston de Hallette, montrant les quatre tubes contenus dans la tige de connexion et le coussin annulaire D autour du piston. Archives nationales F/14 11171.

(c) Archives nationales.

Bricoleur impénitent, Hallette ne cessait pas d'imaginer des améliorations pour les différentes parties de son chemin d'essai, les amenant de fait à une complexité technique assez éloignée de l'inspiration «naturelle» d'origine. Mais les incessants perfectionnements apportés aux organes essentiels - le piston, les clapets d'entrée d'air et les lèvres - apparaissaient comme autant de preuves de sa propre insatisfaction de l'invention, compromettant, à la longue, ses chances de participer à l'essai officiel d'un chemin atmosphérique qui se mettait alors en place. Pour ce dernier, Hallette n'était pas encore à même de fournir des dessins ne varietur de son système ${ }^{195}$. Ainsi, malgré l'appui de l'Académie d'Arras et de la Chambre de Commerce de la ville, des députés du Pas-de-Calais ${ }^{196}$ et de la presse du département, du savant François Arago, de certains membres du Conseil des Ponts et Chaussées et, enfin, de L'Illustration ${ }^{197}$, le système de Hallette, on le verra, ne reçut pas d'autres essais pratiques en France. Dépité, Hallette se résolut à devenir prophète outre-Manche, cédant la propriété de son invention à deux entrepreneurs anglais qui, en mai 1846, fondèrent à Londres Hallette's Atmospheric Railway and Canal Propulsion Company. D'après Hadfield, une ligne expérimentale de 400 pieds ( $122 \mathrm{~m}$ ) fut construite sur le terrain d'un pub à Peckham, en banlieue sud-est de Londres, ligne ouverte au public trois jours par semaine, avec des tickets gratuits disponibles au bureau de la compagnie, à Winchester House, 52 Old 
Broad Street ${ }^{198}$. En juin 1846, dans le cadre de cette affaire, Hallette partit pour Londres accompagné de son secrétaire M. Gibon qui raconta par la suite son accueil chaleureux par les ingénieurs les plus distingués de l'Angleterre, les Cubitt, les Vignole, les Brunel, les Stephenson... ${ }^{199}$ Mais à son retour en France, Hallette tomba malade et mourut le 3 juillet 1846, laissant son usine à son fils unique, Alfred-Augustin. Ce dernier participa à la réalisation du chemin de fer atmosphérique de Saint-Germain en tant que fournisseur de deux de ses machines fixes, mais les lèvres pneumatiques de son père tombèrent dans l'oubli avec le discrédit général du système atmosphérique, après les échecs à Croydon et dans le Devon. Sa santé financière compromise par tant d'expériences coûteuses, l'entreprise arrageoise ne résista pas à la crise de 1847. Elle fut mise en faillite et son matériel vendu en novembre 1847.

\section{NOTES}

1. Sur les origines des chemins de fer en France, voir CARON, François. Histoire des chemins de fer en France, tome $1^{\mathrm{er}}, \mathbf{1 7 4 0 - 1 8 8 3}$. Paris : Fayard, 1997; RIBEILL, Georges. La révolution ferroviaire, la formation des compagnies de chemins de fer en France (1823-1870). Paris : Belin, 1993. ; PALAU, François et Maguy. Le rail en France, les 80 premières lignes, 1828-1851. Paris : F. et M. Palau, 1995. Pour les chemins de fer de la région de Saint-Étienne, voir plus particulièrement COTTE, Michel. Le choix de la révolution industrielle, les entreprises de Marc Seguin et ses frères (1815-1835). Rennes : Presses universitaires de Rennes, 2007.

2. BINEAU, Jean-Martial. Chemins de fer d'Angleterre, leur état actuel, législation qui les régit, conditions d'art de leur tracé, leur mode et leurs frais d'établissement, leur système et leurs frais d'exploitation, leur circulation, leurs tarifs et leurs produits ; application en France des résultats de l'expérience de l'Angleterre et de la Belgique. Paris : Carillan-Gœury et $\mathrm{V}^{\mathrm{ve}}$ Dalmont, 1840.

3. RONCAYOLO, Marcel. Préludes. BOWIE, Karen et TEXIER, Simon (dir.). Paris et ses chemins de fer. Paris : Action artistique de la Ville de Paris, 2003, p. 31-35.

4. LE CHATElieR, L., FLACHAT, E., PETIET, J. et POLONCEAU, C. Guide du mécanicien constructeur et conducteur de machines locomotives. Paris : Paul Dupont, 1851, et atlas de 74 planches, p. 16.

5. MORIN, Général. Machines motrices et moyens locomoteurs. DUPIN, Baron Charles. Exposition universelle de 1851, Travaux de la Commission française sur l'industrie des nations, publiés par ordre de l'Empereur, Tome III, Première partie, Première section. Paris : Imprimerie impériale, 1857, p. 33-34.

6. RIBEILL, Georges. La Révolution ferroviaire, op. cit. (note 1), p. 273-275, et CARON, François. Histoire des chemins de fer en France, op. cit. (note 1), p. 101-102; voir aussi MERCIER, Pierre. L'opinion publique après le déraillement de Meudon en 1842. Paris et île-de-France, Mémoires publiés par la Fédération des Sociétés historiques et archéologiques de Paris et de l'île-deFrance, 1993, tome 44, p. 153-174. Cet auteur fixe le nombre de victimes à 200.

7. Journal des Chemins de fer, 15 mai 1842.

8. Il s'agit, bien évidemment, des craintes de voyageurs ou des propos d'observateurs hostiles aux locomotives. Sur les cheminots, voir VINCENOT, Henri. La vie quotidienne dans les chemins de fer au XIX ${ }^{\mathrm{e}}$ siècle. Paris : Hachette, 1975, et RIBEILL, Georges. Le personnel des compagnies de 
chemins de fer, matériaux pour une contribution à la sociologie historique des professions, 1 - des origines à 1914. Paris: Rapport, Mission de la Recherche, A.T.P. socio-économie des transports, 1980.

9. ZAMBAUX D'AMBLY, J. Description d'un nouveau système de chemin de fer atmosphérique suivie d'une note sur l'emploi de l'air comprimé avec le même mode de locomotion. Paris : Typographie Lacrampe et Cie, s.d. [1845], p. 4.

10. MARECHAL, Virginie. La construction des lignes de chemin de fer de Paris à Rouen et de Rouen au Havre, 1839-1847. Mémoire de maîtrise en histoire. Paris : Université de Paris I, 1993-1994; BOCARD, Hélène, introduction à l'ouvrage collectif. De Paris à la mer, la ligne de chemin de fer Paris-Rouen-Le Havre. Paris : A.P.P.I.F., 2005, p. 9-45. (Images du Patrimoine $n^{\circ} 239$ ).

11. Voir la préface de Louis Bergeron à l'ouvrage d'AUCLAIR, Alain. Les ingénieurs et l'équipement de la France, Eugène Flachat (1802-1873). Le Creusot: Écomusée de la Communauté urbaine Le Creusot-Montceau-les-Mines, 1999, p. 13. Une première version de ce présent article sur les chemins de fer atmosphériques a été présentée dans une session de recherche sur les échanges ferroviaires dans l'Europe du XIX ${ }^{e}$ siècle, organisée par Karen Bowie au colloque «Inventing Europe : Technology and the Making of Europe », tenu à Rotterdam en juin 2007.

12. Papin est mort en 1712 ou en 1714 - on ne sait trop - dans un état "voisin de la misère » (LAROUSSE. Grand dictionnaire universel $\mathrm{du} \mathrm{XIX}^{\mathrm{e}}$ siècle): son «immortalité » est une construction du XIX ${ }^{e}$ siècle. À Paris, des statues de Papin ornent les cours du Louvre et du Conservatoire des arts et Métiers.

13. PAPIN, Denis. Recueil de diverses pièces touchant quelques nouvelles machines et autres sujets philosophiques dont on voit la liste dans les pages suivantes. Cassell : Chez la Veuve de Jean George, imprimeur de la cour, 1695. Papin semble entrevoir ici une notion de «galérienvapeur » mais c'est le horse-power, le « cheval-vapeur » de James Watt, qui s'imposera.

14. L'effervescence philosophique, scientifique et inventive autour de cette manufacture matricielle -spécialisée d'abord dans la quincaillerie fine mais connue surtout pour la construction de machines à vapeur - est bien évoquée dans l'ouvrage de UGLOW, Jenny. The Lunar Men, The Friends who made the Future, 1730-1810. London : Faber \& Faber, 2002. Les très riches archives de la manufacture sont présentées par JONES, Peter M. Les inventeurs et l'activité inventive dans les archives de Soho. COURCY, Marie-Sophie, Douyère-Demeulenaere, Christiane et HILAIRE-PEREZ, Liliane (dir.). Les archives de l'invention, écrits, objets et images de l'activité inventive, Actes du colloque international organisé au Conservatoire national des arts et métiers et au Centre historique des Archives nationales, 26-27 mai 2003. Toulouse : CNRS-Université de Toulouse - Le Mirail, 2006, p. 203-210.

15. GRIFFITHS, John. The Third Man, The Life and Times of William Murdoch, 1754-1839, the Inventor of Gas Lighting. London: André Deutsch, 1992. Cette biographie est une réévaluation de l'importance du rôle de Murdoch dans le succès de l'entreprise formée en 1774 par Boulton et Watt, d'où son titre, d'inspiration cinématographique.

16. THURSTON, R.-H. Histoire de la machine à vapeur, revue, annotée et augmentée d'une introduction par $\mathrm{J}$. Hirsch, professeur de machines à vapeur à l'École des Ponts et Chaussées de Paris. Paris : Librairie G. Baillière et Cie, 1880, p. 138.

17. TRIGER. Mémoire sur un appareil à air comprimé pour le percement des puits de mine et autres travaux sous les eaux et dans les sables submergés. Comptes rendus de l'Académie des Sciences. Séance du mardi 2 novembre 1841, tome XIII, p. 884-896.

18. NEPVEU, Charles. Note sur les fondations en rivière. Mémoires et compte rendu des travaux de la Société des Ingénieurs Civiles. Vol VIII, année 1855, p.173-215. L'auteur qui décrit les travaux en Angleterre sera chargé, en 1858, de la réalisation, à air comprimé, des fondations de la "passerelle » ferroviaire à travers la Garonne à Bordeaux, mieux connue 
aujourd'hui sous le nom de « passerelle Eiffel », Eiffel étant entré dans l'entreprise de Nepveu en 1856.

19. Sur les ponts de Brunel, voir la biographie «classique » de ROLT, L. T. C. Isambard Kingdom Brunel, A Biography. London: Longman, 1957, la biographie «révisionniste » de VAUGHAN, Adrian. Isambard Kingdom Brunel, Engineering Knight Errant. London : John Murray, 1991, et la récente collection d'essais publiée à l'occasion du bicentenaire de la naissance de l'ingénieur: KELLY, Andrew and KELLY, Melanie (eds). Brunel, in Love with the Impossible, a Celebration of the Life, Work and Legacy of Isambard Kingdom Brunel. Bristol: Bristol Cultural Development Partnership, 2006. Ce bicentenaire a également vu la publication d'un guide du patrimoine brunellien, CHRISTOPHER, John. Brunel's Kingdom, in the Footsteps of Britain's Greatest Engineer. Stroud (Gloucestershire) : Tempus Publishing Limited, 2006, ainsi que la réédition de la biographie de l'ingénieur publiée par son fils en 1870 : BRUNEL, Isambard. The Life of Isambard Kingdom Brunel, Civil Engineer. Stroud (Gloucestershire) : Nonsuch Publishing Ltd, 2006, et la publication d'une nouvelle biographie, luxueusement illustrée, de BRINDLE, Steven. Brunel, The Man who Built the World. London : Weidenfeld \& Nicolson, 2005. Sur le tunnel sous la Tamise entre Wapping et Rotherhithe, tunnel emprunté aujourd'hui par une ligne du tube, voir, KENTLEY, Eric (ed.). The Brunels' Tunnel, Rotherhithe, London : The Brunel Museum, 2006.

20. VUIGNER, Émile. Pont sur le Rhin à Kehl (embranchement de Strasbourg à Kehl), Note relative aux travaux de fondations des piles et culées. Paris : Typographie Hennuyer, 1862.

21. MARREY, Bernard. Les ponts modernes, 18 $^{\mathrm{e}} \mathbf{1 9}^{\mathrm{e}}$ siècles. Paris : Picard, 1998, p. 173-178.

22. CORNET, F.-L. Description des machines à air comprimé employées par la société des charbonnages des Sars-Longchamps et Bouvy à St.-Vaast. Sars-Longchamps : (Extrait du $12^{\mathrm{e}}$ Bulletin de l'Association des Anciens Élèves de l'École des Mines du Hainaut), 1865.

23. PERNOLET, M. A. L'air comprimé et ses applications; production, distribution et conditions d'emploi. Paris : Dunod, 1876, p. 1.

24. L'histoire des chemins de fer atmosphériques a fait l'objet de deux ouvrages en langue anglaise dans les années 1960: CLAYTON, Howard. The Atmospheric Railways. Lichfield: published by the author, 1966, et HADFIELD, Charles. Atmospheric Railways, a Victorian Adventure in Silent Speed. Newton Abbot : David \& Charles, 1967 (fig. note 24). François Caron, qui contribua aux recherches pour ce dernier livre, traite du sujet dans son Histoire des chemins de fer en France, op. cit. (note 1), p. 295-298. Plus récemment, l'historien Henry Atmore est revenu sur le dossier en analysant l'échec du système atmosphérique dans le cadre des «intérêts " ferroviaires des années 1840 et de l'effondrement de la "railway mania » en 1845 : ATMORE, Henry. Railway interests and the 'rope of air', 1840-1848. British Journal for the History of Science, septembre 2004, 37 (3). p. 245-279. Sur le chemin de fer atmosphérique français, voir aussi RATCLIFFE, Barrie M. The Building of the Paris - Saint-Germain Railway, some entrepreneurial and financial problems in the launching of railways in France in the 1830s and 1840s. Journal of Transport History, février 1973, p. 20-40, et CARRIERE, Bruno. Le chemin de fer atmosphérique: une expérience sans lendemain. Rail Passion, septembre 1996, $\mathrm{n}^{\circ} 11$, p. 53-59. Plusieurs sites Web évoquent les chemins de fer atmosphériques ou pneumatiques, dont, notamment, celui de BRADER, Mark : http://www.davros.org/rail/atmospheric.html (01/12/08).

25. Les brevets, numéros 2299 et 2431 (dont des copies sont conservées par l'Institution of Civil Engineers à Londres [ICE], J. G. James collection) décrivent une machine susceptible de "condenser le vent» à l'intérieur d'un récipient appelé "magazine». Elle permettrait l'accumulation des forces irrégulières du vent pour les appliquer à d'autres machines ou à des transports pour lesquels on utilise la vapeur, le vent, l'eau ou des chevaux. L'un des avantages de l'engin souligné par l'inventeur consistait dans le fait qu'il évitait la cruauté exercée à l'égard des animaux. 
26. MEDHURST, George. A new method of conveying letters and goods with great certainty and rapidity by air [Une nouvelle méthode pour le transport des courriers et des marchandises avec grande certitude et célérité, par l'air]. London : Printed by D.N. Shury, Soho, 1810.

27. MEDHURST, George. Calculations and remarks tending to prove the practicability, effects and advantages of a plan for the rapid conveyance of goods and passengers upon an iron road through a tube of 30 feet in area by the power and velocity of air [Calculs et remarques tendant à faire la preuve de la faisabilité, des effets et des avantages d'un plan pour le transport rapide des marchandises et des voyageurs sur une route en fer à travers un tube de 30 pieds de surface par la puissance et la vélocité de l'air]. London : D.N. Shury, Soho, 1812.

28. MEDHURST, George. A new system of inland conveyance, for goods and passengers, capable of being applied and extended throughout the country and of conveying all kinds of goods, cattle and passengers, with the velocity of sixty miles in an hour, at an expense that will not exceed the one-fourth part of the present mode of travelling without the aid of horses or any animal power [Un nouveau système de transport terrestre pour des marchandises et des voyageurs, capable d'être appliqué et étendu à travers tout le pays pour le convoyage de toutes sortes de marchandises, du bétail et des voyageurs, à une vitesse de soixante milles en une heure, et pour un coût qui ne dépassera pas le quart des modes de transport actuels, sans recours aux chevaux ni à la traction animale]. London : T. Brettell, 1827.

29. VALLANCE, John. On Facility of Intercourse [Sur la facilité des rapports]. London: W. Clowes, 1824. Exemplaire avec annotations de l'auteur dans les collections de l'ICE. L'ouvrage met en exergue la définition donnée par un dictionnaire imaginaire de l'année 1924 pour le mot « impossible : mot autrement très usité, même par des gens intelligents, mais que l'on considère aujourd'hui comme indiquant une pauvreté d'information, une limitation de l'intellect et une absence de toute grandeur de conception."

30. Dans une brochure de 1827 , rédigée en réponse à un détracteur de son système, Vallance prétend qu'il n'avait, en 1824, aucune connaissance des travaux de Medhurst, publiés en 1812, et que, de toute manière, son système à lui, s'opérant par le vide, se distinguait des projets de Medhurst, marchant à l'air comprimé : VALLANCE, John. A letter to M. Ricardo Esq., in reply to his letter to Dr Yates on the proposed method of pneumatic transmission or conveyance by atmospheric pressure [Lettre au Sieur Ricardo en réponse à sa lettre au docteur Yates sur la méthode projetée de transmission pneumatique ou convoyage par la pression atmosphérique]. Brighton : Creasy and Baker, 1827, (appendix, p. 101-106).

31. Cette visite par le comte de Flahaut, le père du duc de Morny, est attestée par SEGUIN, Marc. Note sur les chemins de fer atmosphériques, en employant comme moteur l'action de l'air dans les tunnels d'une longue étendue, dont la section est égale à l'espace que les convois y occupent. Comptes rendus de l'Académie des Sciences. Séances du 5 juin et du 4 septembre 1854, tome 38, p. 993-998, et tome 39, p. 456-462, p. 994.

32. RICARDO, M. Esq. A letter addressed to Dr. Yates on the proposed plan of travelling by atmospheric pressure. Brighton: C. and R. Sickelmore, 1827, p. 6. C'est cette brochure qui tourne en dérision le projet de Vallance en demandant si, pour économiser quelques heures, un voyageur se laisserait volontiers enfermer dans un tunnel, privé de la lumière du jour, des vues de la campagne environnante et de tous les effets agréables et exhilarants d'un voyage. La première ligne du chemin de fer métropolitain, inaugurée à Londres une trentaine d'années plus tard, donna une réponse à cette question.

33. Le prospectus est publié en annexe de la lettre de Vallance à Ricardo (note 30).

34. PINKUS, Henry. An improved method of or apparatus for communicating and transmitting or extending motive power, by means whereof carriages or waggons may be propelled on railways or common roads and vessels may be propelled on canals [Une méthode améliorée ou appareil pour la communication et le transport ou l'extension de la puissance motrice, par laquelle des voitures ou wagons peuvent être propulsés sur des chemins 
de fer ou sur des routes ordinaires et des vaisseaux mus sur des canaux], 30 août 1834, exemplaire dans la collection J. G. James aux archives de l'ICE. D'autres brevets comportant des améliorations du système sont pris par Pinkus en 1836, 1840 et 1841, le dernier décrivant l'application de son système à l'agriculture, avec les tuyaux d'air raréfié, reliés à une machine fixe et enterrés sous les champs. Pinkus publia un prospectus pour ce système en 1840 : PINKUS, Henry. Prospectus of a new agrarian system. The pneumatic-atmospheric and gasopneumatic railway, common road and canal transit. London : 1840.

35. Brevet $n^{\circ} 7920$, aux noms de Samuel Clegg et Jacob Samuda pour «a new improvement in valves and the combination of them with machinery "; brevet demandé en France le 27 juillet 1838 et accordé le 29 septembre 1838 à James Bonfil, rentier, demeurant à Paris : «brevet d'invention et de perfectionnement de quinze ans pour des soupapes perfectionnées qui combinées avec d'autres dispositions mécaniques permettent d'utiliser pour le transport des chariots sur les rails-routes, une force motrice dérivée de la pression atmosphérique ", archives de l'Institut national de la Propriété industrielle, Paris. On note la traduction directe de railways en « rails-routes».

36. Memoir of Mr. Samuel Clegg. Quarterly Papers on Engineering. London : John Weale, vol II, part III, Eng. III, 1844. Écrit avec les papiers de son père sous les yeux, le traité pratique du fils de Samuel Clegg sur l'éclairage au gaz cite de nombreux exemples de réalisations de son père dans ce domaine : CLEGG, Samuel, Jun. A practical treatise on the manufacture and distribution of coal-gas; its introduction and progressive improvement, illustrated by engravings from working drawings, with general estimates. London : John Weale, 1841.

37. On lui connaît, également, une machine à vapeur portative, inventée vers 1807 : Machine à vapeur portative inventée par M. Samuel Clegg, élève de MM Watt et Boulton, Bulletin de la Société pour l'encouragement de l'Industrie nationale. Juillet 1807, vol. 6, $\mathrm{n}^{\circ}$ XXXVII. p. 8-9 et planche 37 , texte traduit du journal anglais de Nicholson. La machine se distinguait par son nouveau va-et-vient, communiquant le mouvement du piston à une roue par l'intermédiaire de deux crémaillères dentées.

38. HADFIELD, Charles. Atmospheric Railways, op. cit. (note 24), p. 29.

39. Une collaboration entre Clegg et les Samuda est attestée en 1838 autour de l'invention d'un compteur à gaz : Samuda en fit la présentation à la huitième réunion annuelle, à Newcastle, de la British Association for the Advancement of Science: SAMUDA, On Clegg's dry gas-meter, Mechanics' Magazine, $\mathrm{n}^{\circ} 788,15$ septembre 1838.

40. http://www.jewishencyclopedia.com. (01/12/08).

41. Journal des Chemins de Fer, 17 février 1844.

42. Dans sa biographie de son père, Isambard Brunel déclare que la mort de Jacob Samuda en 1844 devait être considérée comme une perte irréparable non seulement pour son frère Joseph mais pour tous ceux concernés, comme l'était alors son père, par la maîtrise des difficultés techniques que présentaient les chemins de fer atmosphériques; BRUNEL, Isambard. op. cit. (note 19), p. 131.

43. SAMUDA, Jacob, The Atmospheric Railway. Institution of Civil Engineers, session 1844, vol III. p. 256-283, session du 14 mai 1844 présidée par William Cubitt. Selon le «Memoir of Mr. Samuel Clegg ", en revanche, les dimensions du tube, la forme de la soupape et les dispositifs imaginés pour son ouverture, sa fermeture et son scellement, revenaient tous à Samuel Clegg, Samuda ayant surtout contribué au perfectionnement de certains détails du système. Son expérience dans l'industrie de l'éclairage au gaz avait certainement donné à Clegg une grande familiarité avec la fabrication et l'installation de tubes en fonte. D'après son fils, les techniques employées pour assurer des joints élastiques étanches entre des sections d'un tuyau à gaz techniques ayant recours à un mélange de suif russe et d'huile végétale ordinaire, coulé entre deux cordes de calfatage - furent directement employées sur les chemins de fer atmosphériques ; CLEGG, Samuel, Jun. op. cit. (note 36), p. 23. 
44. Terme utilisé dans le brevet français de James Bonfil de 1838 ; par la suite on parlera plutôt de wagon directeur.

45. Selon Samuel Clegg fils, un compteur à gaz breveté par son père en 1834 n'était pas utilisé en Angleterre mais était « universellement » adopté à Paris ; CLEGG, Samuel, Jun. op. cit. (note 36), p. 164. Notons ici la présence dans l'industrie parisienne du gaz des entrepreneurs britanniques Aaron Manby et Daniel Wilson, créateurs des forges de Charenton ; en 1821, ils avaient participé à la création d'une usine à gaz installée aux Ternes. Voir BELHOSTE, Jean-François. Les forges de Charenton. CINQUALBRE, Olivier. Architecture d'usines en Val-de-Marne, 1822-1939. Paris : Inventaire général, APPIF et ADAGP, 1988, p. 25-32 (Cahiers de l'Inventaire, $\mathrm{n}^{\circ}$ 12, réimpression en 2004).

46. TEISSERENC, Edmond. Du chemin de fer atmosphérique, La Presse, 7 août 1843. Polytechnicien touché par le saint-simonisme, Teisserenc de Bort avait passé deux ans, de 1835 à 1837, à la toute nouvelle École d'application des manufactures des tabacs, au Gros Caillou, mais, ayant donné sa démission de cette administration en 1837, il s'était intéressé aux chemins de fer, dont il devint un expert reconnu, publiant de nombreux ouvrages à leur sujet au cours des années 1840. Membre du conseil d'administration du PLM sous le Second Empire, il fut ministre de l'Agriculture entre 1872 et 1877, terminant sa carrière comme vice-président du Sénat. Voir LENOBLE, Jean. P.-E. Teisserenc de Bort, gentilhomme limousin, sénateur, ministre, ambassadeur (1814-1892). Limoges : S.E.L.M., 1977.

47. Voir SMITH, Paul. Les pompes à feu, le dossier iconographique. L'Archéologie industrielle en France, décembre 1999, n 35, p. 53-62.

48. PAYEN, Jacques. Capital et machine à vapeur au XVIII ${ }^{\mathrm{e}}$ siècle. Les frères Périer et l'introduction en France de la machine à vapeur de Watt. Paris : Mouton, 1969.

49. Voir CALLITE, Anne. La construction du matériel roulant ferroviaire en France, l'exemple nordiste (1828-1939). Thèse de doctorat en histoire. Lille : Université de Lille III, s.d., [2006], deux tomes, ff 113-114; et PAYEN, Jacques. Technologie de l'énergie vapeur en France dans la première moitié du XIX $^{\mathrm{e}}$ siècle, La machine à vapeur fixe. Paris : C.T.H.S., 1985, p. $90-93$.

50. Comptes rendus hebdomadaires de l'Académie des Sciences. 1839, tome VIII. p. 238-240. À la suite de ce compte rendu, le Bulletin de la Société d'Encouragement pour l'Industrie nationale fit également une présentation des nouveaux chemins de fer pneumatiques dans une notice industrielle de mars $1839, \mathrm{n}^{\circ}$ CCCXII, p. 95-96.

51. Ces premiers essais français sont également mentionnés par Mallet. Rapport sur le chemin de fer établi, suivant le système atmosphérique, de Kingstown à Dalkey, en Irlande, et sur l'application de ce système aux chemins de fer en général. Paris, 10 janvier 1844 ; ainsi que dans Armengaud Aîné, Chemins de fer atmosphériques. Publication industrielle des machines, outils et appareils les plus perfectionnés et les plus récents employés dans les différentes branches de l'industrie française et étrangère. Tome sixième, Paris. 1848, p. 70-194, p. 73. L'entreprise des frères Michel et Claude Nillus - Atelier de construction de machines à vapeur de terre et de mer et autres mécaniques, dock Vauban, au Havre - était l'un des fournisseurs pour la construction en France de la ligne de chemin de fer de Paris à Rouen et au Havre. Sur cette entreprise, voir ÉTIENNE-STEINER, Claire. Le Havre, un port, des villes neuves. Paris : Monum, Éditions du Patrimoine, 2005, p. 297.

52. CLEGG, Samuel. Clegg's Patent Atmospheric Railway. London : Richard Kinder, 1839. 20 p. (Le Mechanics' Magazine en date du 15 juin 1839 cite cette brochure " récemment publiée. »). C'est de ces essais à Southwark dont il s'agit dans une brève communication faite à l'Académie des Sciences dans sa séance du 6 mai 1839: «M. Bonfil annonce qu'il a assisté à Londres à une expérience du nouveau système pneumatique de M. Clegg. ", Comptes rendus de l'Académie des Sciences, 1839, tome VIII, p. 714.

53. D’après HADFIELD, Charles. Atmospheric Railways, op. cit. (note 24), p. 31. 
54. Vitesses bien supérieures aux vitesses moyennes sur les chemins de fer à locomotives de l'époque, d'entre vingt et trente milles à l'heure, selon SCHIVELBUSCH, Wolfgang. The Railway Journey, The Industrialization of Time and Space in the $19^{\text {th }}$ Century. Berkeley and Los Angeles : The University of California Press, 1986, p. 33.

55. Dans une lettre à sa femme en date du 15 mai 1842, Airy décrit sa visite à Wormwood Scrubs en compagnie de l'ingénieur Charles Vignoles, un ingénieur nommé Smith, James Pim, secrétaire de la Compagnie du chemin de fer de Dublin à Kingstown, les frères Samuda et Samuel Clegg ( «l'homme qui a inventé le gaz dans ses dispositions actuelles »); malheureusement, le jour de sa visite, la machine fixe tomba en panne mais il put quand même voir une voiture atmosphérique en mouvement, sans cause visible, comme si elle était conduite par le diable. Pour cet observateur, il n'y avait pas de doute quant à la praticabilité mécanique du système, mais il doutait de sa rentabilité. AIRY, George Biddell. Autobiography. Cambridge: Cambridge University Press, 1896, p. 155-157.

56. Journal des Chemins de Fer, 3 décembre 1842.

57. HERARD, E. Railway atmosphérique, ou emploi de la pression atmosphérique comme force motrice sur les chemins de fer. Annales des Ponts et Chaussées. 1843, $2^{\mathrm{e}}$ série, Mémoires, Tome V, premier semestre 1843, p. 47-77. L'auteur utilise comme source principale la brochure publiée en mars 1841 par Joseph Samuda: SAMUDA, Joseph d'Aguilar. A Treatise on the Adoption of Atmospheric Pressure to the Purposes of Locomotion on Railways. London: John Weale, 1841. (exemplaire dans AN F 14 11171). Cette brochure fut publiée en langue française, en 1842 : SAMUDA, Joseph d'Aguilar. Railways atmosphériques ou application de la pression atmosphérique à la traction sur les railways, par Samuda, traduit et annoté par $\mathrm{C}$. du Perron. Paris : L. Mathias, 1842, 48 p.

58. Teisserenc fut accompagné à Wormwood Scrubs par l'ingénieur d'origine irlandaise Charles Vignoles, l'un des principaux promoteurs du nouveau système atmosphérique, mieux connu en France pour ses rails en forme de T inversé ; voir VIGNOLES, K. H. Charles Blacker Vignoles : romantic engineer. Cambridge : Cambridge University Press, 1982. 187 p. (note p. 176 sur la visite de Teisserenc de Bort). Sur place, Teisserenc eut également des contacts avec Thomas Bergin, venu examiner la ligne expérimentale pour le compte de la compagnie de Dublin à Kingstown.

59. TEISSERENC, Edmond. Rapport adressé à M. le Ministre des Travaux Publics sur les chemins de fer. Paris Imprimerie royale, 1843, p. 119. Voir notamment la troisième partie, Rapport à M. le Ministre secrétaire d'État des Travaux publics, sur le nouveau mode de locomotion, dit système atmosphérique. p. 105-140. Ce rapport, qui ne semble pas être conservé à la BnF, peut être consulté dans le fonds Chemins de fer du Centre de ressources documentaires, administratives et juridiques du ministère de l'Écologie, du développement et de l'aménagement durables (Grande Arche de la Défense, cote CHEM 0400).

60. Depuis novembre 1841, Charles Vignoles était professeur de «Civil Engineering " à l'University College de Londres. Dès sa conférence inaugurale, dont le Mechanics' Magazine du 13 novembre 1841 fit un compte rendu, il parla du nouveau système atmosphérique, convaincu de son imminente application, étendue et lucrative.

61. Mechanics' Magazine, 9 juillet et 12 novembre 1842, et Journal des Chemins de Fer, 9 juillet et 5 novembre 1842 .

62. TEISSERENC, Edmond. Du chemin de fer atmosphérique, La Presse, 7 août 1843.

63. Rebaptisée Dublin Pearse Station en 1966, du nom de Patrick Pearse, l'un des dirigeants de l'insurrection de Pâques 1916, exécuté par les autorités militaires britanniques.

64. Dún Laoghaire de nos jours, ou Dunleary dans sa forme anglicisée. Le nom de Kingstown date de la visite du roi George IV en 1821. Voir IRELAND, John de Courcy. History of Dun Laoghaire Harbour. Dublin : Caisleán an Bhúrcaigh, 2001. 
65. Assemblée générale ordinaire de la Compagnie Dublin et Kingstown, 25 novembre 1831, archives de la compagnie conservées par Córas Iompair Éirann, Heuston station, Dublin. Sur l'histoire de la compagnie de Dublin \& Kingstown, voir MURRAY, K. A. Ireland's First Railway. Dublin : Irish Railway Record Society, 1981.

66. BERGIN, Thomas F. The Atmospheric Railway, Observations on the report of Lieut. Colonel Sir Frederick Smith, R. E. and Professor Barlow, on the Atmospheric Railway. Dublin : Hodges and Smith, 1843. [ICE].

67. PIM, James. Irish Railways, The Atmospheric Railway, A Letter to the Right Hon. Lord Viscount Morpeth. London : J. L. Cox \& sons, 1841, et PIM, James. The Atmospheric Railway, A Letter to the Right Hon. The Earl of Ripon, President of the Board of Trade. London : Printed for private circulation, 1841. Une deuxième édition de cette brochure, datée du 24 juin 1842, se trouve dans AN F 1411171.

68. Memorial of Samuel Clegg and Jacob Samuda to the Right honourable the Lords Commissioners of Her Majesty's Treasury, 10 June 1841, House of Commons, Copies of Correspondence and Papers relative to the Dublin and Kingstown Railway. London : ordered to be printed by the House of Commons, 28 February 1843, [ICE].

69. Dans son rapport du 19 mars 1843, Edmond Teisserenc donne les détails suivants sur le marché signé entre les inventeurs et la compagnie. Voir le fichier en annexe. « Ce qui fait, par mètre courant, cent francs en nombre rond.». TEISSERENC, Edmond. Rapport adressé à M. le Ministre des Travaux Publics sur les chemins de fer. op. cit. (note 59), p. 139.

70. Mechanics' Magazine, 19 March 1842.

71. Dublin and Kingstown Railway, assemblées des actionnaires des 6 avril 1842 et 29 mars 1845, archives Córas Iompair Éirann, Dublin.

72. William Fairbairn (1789-1874), l'un des plus célèbres constructeurs mécaniques de l'époque, est surtout connu en France pour ses bâtiments industriels « fireproof » à poutres et poteaux en fonte. Il est notamment le concepteur de la célèbre filature de coton dite «La Foudre ", édifiée au Petit-Quevilly, près de Rouen, en 1846.

73. Un dessin de cette machine, signé « Samuda Bros. Engineers, London, May 1843 », se trouve dans AN F14 11171.

74. Du moins selon le Dublin Evening Mail, cité par le Mechanics' Magazine en date du 4 novembre 1843 .

75. En 1849 , ces tarifs furent abaissés à $2 \mathrm{~d}$ en deuxième classe et $1 \mathrm{~d}$ en troisième classe, $1 \mathrm{~d}$ étant à l'époque, à titre de comparaison, à peu près le prix d'une pinte de Guinness.

76. Dublin and Kingstown Railway, assemblée générale des actionnaires du 27 mars 1844, archives Córas Iompair Éirann, Dublin.

77. The Dublin Evening Mail, cité par le Mechanics' Magazine du 4 novembre 1843. La rédaction de cette dernière revue précisait que les mérites de l'invention étaient généralement attribués à Samuel Clegg.

78. The Dublin Evening Post, 31 octobre 1843, cité par le Mechanics' Magazine du 11 novembre 1843. Coupure de presse également dans AN F14 11171.

79. Témoignage de Jacob Samuda, Système atmosphérique, Enquête devant la commission de la chambre des Communes pour le chemin de fer de Londres à Epsom, accompagnée du rapport de $M$. Stephenson sur le chemin de fer de Dalkey à Kingstown, et de plusieurs lettres en réponse à ce rapport, traduits de l'anglais par $\mathrm{M}$. Alphonse Lauvray et publié par le Journal des Chemins de Fer. Paris : Journal des Chemins de Fer, 1844, p. 28. Dans les archives de la compagnie (registre de minutes de courrier, 1844-1847), il est question aussi d'une pétition de la veuve Read, le 4 avril 1845 : son mari avait été tué sur la ligne de Dalkey.

80. ROLT, L.T.C. Isambard Kingdom Brunel, op. cit. (note 19), p. 165.

81. Suivant les calculs de Thomas Bergin, le coût d'exploitation par mille sur la ligne atmosphérique était de $8 \frac{1}{4} \mathrm{~d}$ (environ 53 centimes par kilomètre) comparé à $11 \mathrm{~d}$ ( 70 centimes par 
kilomètre) sur la ligne à locomotives entre Dublin et Kingstown. SAMUDA, Jacob, The Atmospheric Railway. Institution of Civil Engineers. 1844, vol. III, session du 14 mai 1844, p. 276. 82. Dans son assemblée générale du 29 mars 1845, la compagnie annonça que pendant les onze premiers mois de son exploitation, l'extension atmosphérique entre Kingstown et Dalkey avait transporté 240225 passagers au total. En 1846, 240731 passagers furent transportés par les trains atmosphériques, mais les chiffres commençaient à baisser l'année suivante, conséquence de « la terrible calamité » dont souffrait le pays, c'est-à-dire la famine résultant de la maladie de la pomme de terre. Mais le nombre de voyageurs se rétablissait en 1849 pour atteindre 342471 en 1851. Voir le bilan chiffré de l'exploitation de la ligne, dans la conclusion de cet article. Les actionnaires exprimaient leur satisfaction à James Pim en lui accordant, le 16 septembre 1844, la somme de mille livres - l'équivalent de deux années de ses rétributions en tant que trésorier - en reconnaissance de ses services en rapport avec le chemin atmosphérique. Archives de la compagnie, Córas Iompair Éirann, Dublin.

83. ROLT, L. T. C. George and Robert Stephenson, the Railway Revolution. London: Penguin Books, 1978 [première édition en 1960], p. 282.

84. WEBSTER, N. W. Joseph Locke : Railway Revolutionary. London : Allen \& Unwin, 1970.

85. «La chose la plus zigzaguant, étroite et disgracieuse que j'ai jamais vue », BROOKE, David (ed.). The Diary of William Mackenzie, the First International Railway Contractor. London : Thomas Telford, 2000, p. 336.

86. Le tronçon à forte pente entre Camden et la gare d'Euston, installée en 1837 au bout de la ligne de Birmingham à Londres, et une autre ligne de six kilomètres, à Blackwall, qui fonctionna de 1840 à 1849 avec un système de câble continu sous les voies, auquel les wagons s'accrochaient. Cette dernière ligne, étudiée par Edmond Teisserenc lors de sa mission d'octobre 1842, avait sept stations intermédiaires et fut l'une des premières à utiliser le télégraphe électrique de Cook et Wheatstone pour coordonner ses opérations. Voir BAILEY, Michael R. Robert Stephenson, The Eminent Engineer. London : Ashgate, 2003, p. 193-195.

87. Témoignage de Robert Stephenson, 20 mai 1844, Système atmosphérique, enquête devant la commission de la chambre des Communes, op. cit. (note 79), p. 75.

88. BERKLEY, George. The peculiar features of the atmospheric railway system. Institution of Civil Engineers, session du 15 avril 1845, vol. IV, p. 251-261.

89. STEPHENSON, Robert. Report on the Atmospheric Railway System. London: John Weale, 1844 (rapport daté du 9 avril 1844). De longs extraits de ce rapport furent publiés dans le Mechanics' Magazine du 15 juin 1844.

90. Témoignage de Robert Stephenson, 20 mai 1844, Système atmosphérique, Enquête devant la commission de la chambre des Communes pour le chemin de fer de Londres à Epsom, op. cit. (note 79), p. 85. Voir ci-après sur cette enquête.

91. TURNBULL, William. An essay on the air pump and atmospheric railway, containing formulae and rules for calculating the various quantities contained in Mr. Robert Stephenson's report on atmospheric propulsion, for the directors of the chester and Holyhead railway company. London : John Williams \& $C^{\circ}, 1847$.

92. Voir la lettre de Jacob Samuda adressée au Railway Times le 3 mai 1844 et celle de Samuel Clegg, au même journal, le 5 juin 1844, traduites dans Système atmosphérique, enquête devant la commission de la chambre des Communes, op. cit. (note 79), p.39-51. Jacob Samuda s'opposait de nouveau à Stephenson, critiquant les déductions erronées tirées de ses observations expérimentales, lors du débat organisé par l'Institution of Civil Engineers le 15 avril 1845, à la suite de la lecture de la conférence donnée par George Berkley (l'un des associés de Stephenson) intitulé The peculiar features of the Atmospheric Railway System. Institution of Civil Engineers, 1845, vol. IV, session du 15 avril 1845, p. 251-290, p. 275.

93. HADFIELD, Charles. Atmospheric Railways, op. cit. (note 24), p. 44. 
94. Suite à un litige avec certains habitants de la ville, qui protestaient que la tranchée de la nouvelle ligne les empêchait d'accéder librement au port, James Walker, en tant qu'ingénieur éminent, avait été invité à Kingstown pendant la construction du chemin. Sa solution au problème consistait en la couverture partielle de la tranchée avec des plaques de fonte recouvertes d'argile et de graviers. MURRAY, K. A., op. cit. (note 65), p. 49.

95. Institution of Civil Engineers. 1844, vol. III, session du 16 janvier 1844, p. 29.

96. HADFIELD, Charles. Atmospheric Railways, op. cit. (note 24), p. 45.

97. William Cubitt cité par James Pim, lettre du 22 novembre 1841 adressée à Joseph Kincaid, président de la compagnie de Dublin à Kingstown, House of Commons. Copies of Correspondence and Papers relative to the Dublin and Kingstown Railway, op. cit. (note 68), p. 8.

98. Dans un article du 7 juin 1845, L'Illustration décrit ces courses d'Epsom, le Chantilly de l'Angleterre : toute la population de Londres - aristocratie, bourgeoisie et peuple - s'y rendait, à pied, à cheval ou en voiture : «On y construit en ce moment un chemin de fer atmosphérique. Dès l'année prochaine, tous les rangs seront égaux... devant le vide.»

99. L'Illustration du 25 octobre 1845 publia une gravure de ce viaduc en demandant «en présence de pareils monuments du génie de notre époque, qui nierait encore le progrès continu?»

100. HADFIELD, Charles. Atmospheric Railways, op. cit. (note 24), planche IX, face p. 80.

101. Contrairement aux conseils de Samuda, qui préconisait le fer forgé, les machines fixes fournies par la célèbre firme de Maudslay Son \& Fields, de Lambeth, comportaient des pièces mobiles en fonte, cassant plus facilement. Voir CLAYTON, Howard. op. cit. (note 24), p. 66.

102. La réputation de William Cubitt ne semble pas avoir été compromise par cet échec. Il fut élu président de l'Institution of Civil Engineers en 1850 et, en 1851, il fut anobli pour sa contribution, en tant que consultant, à la construction du Crystal Palace de Paxton.

103. Mechanic's Magazine, $n^{\circ} 1240,15$ mai 1847.

104. HADFIELD, Charles. Atmospheric Railways, op. cit. (note 24), p. 140.

105. The Illustrated London News, $1^{\mathrm{er}}$ mars 1845 , article illustré d'une gravure d'une des gares en construction sur la ligne de Croydon.

106. Des éléments brunelliens sur cette ligne figurent sur la liste des sites retenus en GrandeBretagne pour une inscription au patrimoine mondial de l'UNESCO. Voir FALCONER, Keith. The Great Western World Heritage Site: The genesis of modern transport. London: English Heritage, 2006.

107. ROLT, L. T. C. Isambard Kingdom Brunel, op. cit. (note 19), p. 117.

108. L'idée d'exploiter des chutes d'eau pour faire le vide dans le tube était également envisagée par Jacob Samuda, si le territoire traversé s'y prêtait. Voir sa présentation du 14 mai 1844 à l'Institution of Civil Engineers, op. cit. (note 43), p. 257. Sur les projets ferroviaires italiens de Brunel, voir BUCHANAN, Angus. Brunel, The Life and Times of Isambard Kingdom Brunel. London : Hambledon Continuum, 2006, chapitre 6, p. 85-96.

109. Selon les calculs faits par Gooch à Dalkey concernant la puissance requise pour faire fonctionner le système atmosphérique, le système à locomotives serait bien moins coûteux. Il ne comprenait pas l'enthousiasme de Brunel pour ce système «certainement la plus grande maladresse [blunder] commise dans les chemins de fer. » $\mathrm{GOOCH}$, Sir Daniel. Memoirs and Diary, transcribed from the original manuscript and edited with an introduction and notes by Roger Burdett Wilson. Newton Abbot : David \& Charles, 1972, p. 46-47.

110. Appelé Newton Abbot depuis 1877.

111. BRUNEL, Isambard. The Life of Isambard Kingdom Brunel, Civil Engineer, op. cit. (note 19), p. 108-112. Sur «l'escapade atmosphérique» de Brunel, voir aussi BUCHANAN, R. A. The Atmospheric Railway of I. K. Brunel. Social Studies of Science, mai 1992, vol. 22, n 2. p. 231-243 (actes d'un symposium ICOHTEC sur « failed innovations »). Cet article est repris dans le chapitre 
«Disasters " de la biographie de Brunel par BUCHANAN, Angus. Brunel, The Life and Times of Isambard Kingdom Brunel, op. cit. (note 108), p. 103-112.

112. South Devon Railway, First General Meeting of Proprietors, 28 août 1844, exemplaire dans AN F 148642.

113. South Devon Railway, Second half-yearly General Meeting of Proprietors, 12 février 1845, rapport de l'ingénieur en date du 8 février, AN F 14 8642. Boulton et Watt étant décédés, la célèbre firme de Soho était dirigée à cette époque par James Watt (le fils du fondateur), Blake et Garland.

114. Voir les souvenirs de ce voyage racontés par J. C. Horsley, son beau-frère : BRUNEL, Isambard. The Life of Isambard Kingdom Brunel, op. cit. (note 19), p. 371-374.

115. Sur les goûts très éclectiques de Brunel en matière d'architecture ferroviaire, voir BRINDLE, Steven, op. cit. (note 19), introduction. Dans son étude des cheminées industrielles en Angleterre, James Douet note que Brunel était le premier à adopter le style italien pour ses cheminées, ce style devenant par la suite, et notamment dans les années 1860 et 1870, assez répandu dans l'architecture usinière. DOUET, James. Going up in smoke. The history of the industrial chimney. London: The Victorian Society, 1988 (photocopie communiquée par Frédéric Pillet). Voir aussi JONES, Edgar. Industrial architecture in Britain, 1750-1939. London : B. T. Batsford Ltd., 1985.

116. Baude, Louis-Alphonse. Note sur le chemin de fer atmosphérique de South-Devon (Devonshire) en Angleterre. Bulletin de la Société d'Encouragement pour l'Industrie nationale. $1^{\mathrm{er}}$ septembre $1847, \mathrm{n}^{\circ}$ DXIX, p. 539-544. Louis-Alphonse Baude, ingénieur en chef des Ponts et Chaussées, visita la ligne de Brunel pendant l'été 1847, en compagnie de Joseph Samuda.

117. GILL, Thomas. Address to the Proprietors of the South Devon Railway. London: Effingham Wilson, 1848, p. 18 [ICE].

118. GILL, Thomas. Address to the Proprietors of the South Devon Railway. London: Effingham Wilson, 1848, p. 19 [ICE].

119. BUCHANAN, R. A. The Atmospheric Railway of I. K. Brunel, article cité (note 111), p. 240. Une technologie nouvelle pleine de promesses, mais vaincue par des rats : on comprend l'attrait de cette explication, aussi légendaire qu'elle soit.

120. BRUNEL, Isambard. The Life of Isambard Kingdom Brunel, op. cit. (note 19), p. 121.

121. D'après le Mechanics' Magazine du 11 septembre 1847, les coûts d'installation atteignaient déjà $€ 10000$ livres par mille, environ $156000 \mathrm{~F}$ par kilomètre.

122. South Devon Railway, assemblées générales des 28 février et 29 août 1848, AN F 148642.

123. "As wild a visionary as ever existed », HADFIELD, Charles. Atmospheric Railways, op. cit. (note 24), p. 168.

124. BRUNEL, Isambard. The Life of Isambard Kingdom Brunel, op. cit. (note19), p. 132.

125. GILL, Thomas. Address to the Proprietors of the South Devon Railway, op. cit. (note 117).

126. Sur les différents projets pour cette ligne de Paris à Meaux, dont le premier - pour un «train de vidanges » reliant la capitale à la nouvelle voirie de Bondy - datait de 1837, voir BOWIE, Karen. Expertise et aménagement en Région parisienne au XIX ${ }^{\mathrm{e}}$ siècle. Les enquêtes sur les projets pour une ligne Paris-Meaux, 1838-1841. Histoire Urbaine, décembre 2005, n 14, p 99-122; sur la carrière de Vuigner, voir la notice nécrologique que lui consacre PERDONNET A. dans Mémoires et compte rendu des travaux de la Société des ingénieurs civils. 1865, vol. 18, p. 530-536.

127. VUIGNER, Émile. Mémoire relatif à l'établissement du chemin de fer de Paris à Strasbourg, dans le système atmosphérique ou dans le système mixte de locomotion. Paris : Imprimerie de Mme de Lacombe, 1844 (mémoire daté du 25 avril 1844).

128. Sur les trains articulés d'Arnoux, voir CANDILLON, J.-M. La ligne de Sceaux, des origines à 1871. Mémoire de maîtrise en histoire. Paris: Université de Paris IV, 1980; SMITH, Paul. L'embarcadère de Sceaux. LANGLOIS, Gilles-Antoine (dir.). Montparnasse et le XIV 
arrondissement. Paris: Action artistique de la Ville de Paris, 2000, p.107-112 et CARON, François. La ligne de Sceaux, laboratoire de la «science ferroviaire». Revue d'Histoire des Chemins de Fer, $\mathrm{n}^{\circ}$ 38, printemps 2008, p. 166-189.

129. AUCLAIR, Alain. op. cit. (note 11), p. 96-136 sur le chemin de fer atmosphérique.

130. Assemblée générale extraordinaire de la Compagnie de Saint-Germain, 8 novembre 1844, reproduite dans VILLEDEUIL, Pierre-Charles-Laurent de. Cuvres de Émile et Isaac Pereire, rassemblées par Pierre-Charles-Laurent de Villedeuil et augmentées d'une introduction, d'une biographie des auteurs, de remarques, de tables et d'un choix de documents contemporains, Série G, documents sur l'origine et le développement des chemins de fer (1832-1870). Paris : Librairie Félix Alcan, 1920, vol. I (1832-1845), p. 507-511.

131. Voir LENOBLE, Jean. op. cit. (note 46), p. 36.

132. Dossier individuel de Jacques Mallet (1787-1869), AN F14 2274/2.

133. Il s'agissait encore de Teste, remplacé par Dumon en décembre 1843.

134. Brouillon de lettre, 3 novembre 1843, dossier individuel de Jacques Mallet, AN F 14 2274/2.

135. Mallet. Chemin de fer atmosphérique, rapport adressé à $M$. le ministre des travaux publics, Paris, 28 novembre 1843, reproduit dans FOUQUIER, A. et DESPREZ, H. Annuaire historique universel ou histoire politique pour 1843. Paris: Thoisnier Desplaces, 1844, p. 389-392.

136. Selon un article dans Le Moniteur Industriel du 7 décembre 1843, parlant de Brunel comme étant « le célèbre ingénieur anglo-français »; coupure de presse dans AN F 1411171.

137. " The Lord Mayor requests the honour of M. Mallet's company at dinner on Tuesday the $14^{\text {th }}$ of November at 7 o'clock ", AN F 1411171.

138. Sa mission, prise en charge sur les fonds affectés par la loi du 11 juin 1842 aux études de chemins de fer, revenait à $2244 \mathrm{~F}$, dont 2028 en frais de transport (à un franc par kilomètre) et 18 jours de frais journaliers, à $12 \mathrm{~F}$ la journée (note de frais du 27 novembre 1843). On peut signaler ici que Mallet fut promu au grade d'inspecteur divisionnaire le 28 janvier 1844 . AN F $142274 / 2$

139. Mallet, Report on the Railroad Constructed from Kingstown to Dalkey in Ireland, Upon the atmospheric system and upon the application of this system to railroads in general. Quarterly Papers on Engineering, edited and published by John Weale, London, 1844, vol II, p. 3-55.

140. Cette erreur, répétée ensuite par presque tous les auteurs français, vient peut-être du fait que l'adresse londonienne de l'établissement de Medhurst était 1, Denmark Street. Pour Amédée Guillemin, plus tard, Medhurst était suédois. GUILLEMIN, Amédée. Les chemins de fer. Paris : Librairie Hachette et Cie, 1876, p. 304.

141. WILLIOT, Jean-Pierre. Jules Petiet, Un ingénieur de la première industrialisation et ses réseaux, 1813-1871. Pantin : (c) Indivision Henri M. Petiet, s.d. [2004], ouvrage hors commerce.

142. Sur ce " cabinet d'ingénierie », voir BELHOSTE, Jean-François. La gare Saint-Lazare, terrain exceptionnel des débuts de la construction métallique en France. Revue d'Histoire des chemins de fer, printemps-automne 1999, n 20-21, p. 161-173 ; BELHOSTE, Jean-François. Le berceau de l'ingénierie ferroviaire. BOWIE, Karen et TEXIER, Simon (dir.). Paris et ses chemins de fer, op. cit. (note 3), p. 52-61.

143. REPECAUD. Mémoire sur l'emploi de la pression atmosphérique sur les chemins à rails, lu à la Société académique d'Arras dans ses séances du 19 juillet et 2 août 1844 par le colonel Répécaud, chancelier de cette académie. Arras : Imprimerie de Jean Degeorge, 1844. (extrait des Mémoires de l'Académie d'Arras de 1844).

144. REPECAUD. Mémoire sur l'emploi de la pression atmosphérique sur les chemins à rails, lu à la Société académique d'Arras dans ses séances du 19 juillet et 2 août 1844 par le colonel Répécaud, chancelier de cette académie. Arras : Imprimerie de Jean Degeorge, 1844. (extrait des Mémoires de l'Académie d'Arras de 1844), p. 21-22. Comme Stephenson, dont il semble connaître le rapport, Répécaud pense néanmoins que le système atmosphérique peut 
jouer un rôle, mais seulement sur de courtes rampes, remplaçant la traction à câble. Pour une telle application, Répécaud met en avant son propre système, utilisant un tube sans ouverture longitudinale, dans lequel le piston lui-même serait attaché à la corde de traction.

145. Bulletin de la Société d'Encouragement pour l'Industrie nationale, août 1844, $\mathrm{n}^{\circ}$ CCCCLXXXII, p. 323-324.

146. HADFIELD, Charles. Atmospheric Railways, op. cit. (note 24), p. 214-222.

147. Discours prononcé par François Arago dans la séance de la Chambre des députés du 2 juillet 1844, lors du débat sur le chemin de fer de Paris à Strasbourg (Moniteur Universel, 4 juillet 1844). Citant les avis favorables au système atmosphérique exprimés en Angleterre par Brunel, Cubitt et Vignoles, et, en France, par Teisserenc, Mallet et Vuigner, Arago pense que ceux-ci contrebalançaient très largement celui du seul ingénieur hostile au système, dont la vie tout entière, d'ailleurs, s'était passée au milieu des locomotives. Arago reprend dans ce discours les arguments développés en Irlande quant à l'utilité d'une grande distribution de machines à vapeur sur tous les points du territoire, machines qui, n'ayant pas besoin de travailler toute la journée pour faire le vide dans le tube propulseur, pourraient servir, comme des centrales, pour moudre le blé, pour alimenter en eau les villages voisins, pour les irrigations, etc. Il annonce que pour les $4000 \mathrm{~km}$ de chemins de fer projetés en France d'après la loi du 11 juin 1842, l'emploi du système atmosphérique contribuerait à une économie de 200 à 300 millions de francs: « Mouvement d'étonnement et d'hilarité ».

148. POLONCEAU, Antoine-Rémy. Projet d'un chemin de fer de Dijon à Genève, par Dole, Belmont, Vaudrey, Arbois, Champagnole, Les Planches, Foncine, Bellefontaine, Les Rousses, Mijoux, un souterrain à la Faucille et Gex, mémoire en date du 22 février 1845 rédigé par Antoine-Rémy Polonceau, inspecteur divisionnaire des Ponts et Chaussées en retraite. AD Jura, Sp 2293 (document communiqué par Laurent Poupard). Sur Polonceau, qui avait participé en tant qu'inspecteur divisionnaire des Ponts et Chaussées aux études relatives au chemin de fer de Paris à Orléans, mais qui fut mis à la retraite en 1839 en raison de sa surdité, voir son dossier personnel, AN F 14 2302/2.

149. Il semblerait qu'Andraud ait eu, dès 1830, un encouragement officiel pour ses recherches : le 14 février 1847, le ministre des Finances écrivit au ministre des Travaux publics pour lui demander quel degré d'importance il attachait aux travaux de cet ingénieur, celui-ci étant encore redevable au Trésor de $43000 \mathrm{~F}$, montant d'un prêt consenti en 1830. Voir le sommier des chemins de fer, cité par VILLEDEUIL, Pierre-Charles-Laurent de. Guvres de Émile et Isaac Pereire, op. cit. (note 130), vol. I., p. 1994.

150. ANDRAUD, Antoine. De l'air comprimé employé comme moteur ou de la force obtenue gratuitement et mise en réserve. Paris : chez Guillaumin, 1839; ANDRAUD, Antoine. De l'air comprimé et dilaté comme moteur, ou des forces naturelles recueillies et mises en réserve, seconde édition augmentée d'une partie expérimentale en collaboration avec M. Tessié du Motay. Paris : chez Guillaumin, 1840.

151. Rappelons ici un autre grand enthousiasme de l'époque pour les puits artésiens forés à la sonde, l'un des domaines où étaient actifs Eugène Flachat et son frère Adolphe entre 1827 et 1832. $\mathrm{Au}$ moment où Andraud écrit, le célèbre puits artésien de Grenelle, foré depuis 1833 par le serrurier-mécanicien Georges Mulot, était, à $548 \mathrm{~m}$ de profondeur, sur le point d'aboutir : voir, à ce sujet, SMITH, Paul. Fontaines jaillissantes « artésiennes ». MASSOUNIE, Dominique, PREVOSTMARCILHACY, Pauline et RABREAU, Daniel (dir.). Paris et ses fontaines de la Renaissance à nos jours. Paris : Délégation à l'Action artistique de la Ville de Paris, 1995, p. 199-204.

152. Cette idée d'Andraud d'appliquer l'air comprimé à l'acoustique est mentionnée par GAUGAIN, A.-P.-H. Notice historique sur l'emploi de l'air comprimé considéré comme force motrice et comme agent de locomotion, comprenant l'histoire des diverses applications qui en ont été faites et des nombreuses expériences dont il a été l'objet depuis 1795 jusqu'à nos jours. Paris: Au bureau du Journal des Mines, 1858, p. 77. Pour cet auteur, Andraud avait bon 
droit de réclamer l'honneur d'avoir, le premier, mis en lumière la puissance dynamique de l'air. Il le décrit prêchant la nouvelle doctrine avec une foi d'apôtre et une persévérance de missionnaire.

153. ANDRAUD, Antoine. Chemins à vent ou locomotion par l'air comprimé (système Andraud). Paris : chez Guillaumin, 1846.

154. On entend dans cette formulation un écho de la devise du système métrique décimal, appliqué en France par la loi du 4 juillet 1837.

155. "Système atmosphérique français par l'air comprimé ", courrier d'Andraud adressé au Journal des Chemins de Fer, 22 juin 1844. Pour Andraud, toutefois, malgré sa valeur universelle, l'énergie naturelle de l'air comprimé était également une question d'indépendance nationale. Avec la seule énergie des machines à vapeur, «l'Angleterre, le pays le moins mal pourvu de charbon, fera la loi aux états de l'Europe, elle règnera sur le continent par ses chemins de fer (car ils seront à elle) comme sur les mers par ses bâtiments à vapeur. "

156. Comptes rendus de l'Académie des Sciences, séance du $1^{\text {er }}$ février 1841, tome XII, p. 262.

157. Ministère de l'Agriculture, du Commerce et des Travaux Publics. Description des machines et procédés consignés dans les brevets d'invention, de perfectionnement et d'importation, dont la durée est expirée, et dans ceux dont la déchéance a été prononcée. Paris : Madame Veuve Bouchard-Huzard, 1856, tome LXXXVI, p. 265-272: brevet d'invention n ${ }^{\circ} 11847$ de 15 ans en date du 11 mai 1840, aux sieurs Andrau et Tessier du Montay (sic).

158. L'Illustration du 14 avril 1849 donna des gravures de la roue fluviale et de la turbine éolique imaginées par Andraud.

159. Andraud et Tessié du Motay. Mémoire sur une voiture mise en mouvement par le ressort de l'air. Comptes rendus de l'Académie des Sciences, séance du lundi 17 mai 1841, tome XII, p. 894-899. En avril et mai 1842, Andraud et Tessié du Motay s'adressèrent à l'administration des Ponts et Chaussées avec une soumission pour la construction d'une « locomotive atmosphérique, roues comprises » qu'ils proposèrent pour le chemin de fer de Lille ; voir le sommier des chemins de fer, cité par VILLEDEUIL, Pierre-Charles-Laurent de. Guvres de Émile et Isaac Pereire, op. cit. (note 130), vol. I, p. 1413 et 1415.

160. Journal des Chemins de Fer, 31 août 1844.

161. L'Illustration, 2 novembre 1844.

162. Sur le système de Mékarski, voir notamment les communications de l'inventeur à la société des ingénieurs civils : Mémoires et compte rendu des travaux de la Société des ingénieurs civils, 1876, vol.29, p.107-114 et 1878, vol.31, p.549-555. Une automotrice Mékarski à air comprimé, construite en 1879 et retirée de la circulation en 1917, est conservée par l'AMTUIR dans son dépôt à Colombes, en attendant son transfert à Chelles. Sur ce thème, voir aussi le site consacré aux tramways à air comprimé réalisé par John Prentice :

http://www.tramwayinfo.com/Tramframe.htm ?http://www.tramwayinfo.com/tramways/ Articles/Compair.htm (20/01/09)

163. ANDRAUD, Antoine. Chemins à vent ou locomotion par l'air comprimé (système Andraud). Paris: chez Guillaumin, 1846; et ANDRAUD, Antoine. Chemins éoliques ou locomotion par l'air comprimé. Paris : chez Guillaumin, 1847. C'est apparemment ce système suspendu que Polonceau pensait approprié à la ligne dans le Jura. Andraud précise, en effet, qu'à la campagne, en jouant sur la hauteur des poteaux, il n'y aurait besoin ni de ponts, ni de viaducs, ni de travaux d'art considérables.

164. L'Illustration, 14 avril 1849.

165. Armengaud Aîné, Chemins de fer atmosphériques. Publication industrielle des machines, outils et appareils les plus perfectionnés..., op. cit. (note 51), p. 124.

166. L'Illustration, 16 août 1845.

167. Armengaud se plaint d'ailleurs que la durée des séances accordées pour la consultation des dossiers ne suffisait pas toujours pour leur faire justice : un brevet demandé par Pinkus le 13 mai 
1845 , et cherchant à réunir toutes sortes de procédés connus ou nouveaux, comprenait 182 pages et 49 dessins, la plupart de format grand-aigle, p. 102.

168. «Rapport de Mr Mallet sur les procédés présentés par Mr Geoffroy pour être appliqués aux chemins de fer atmosphériques et sur une machine locomotive à réaction ", séance du Conseil des Ponts et Chaussées du 22 septembre 1845, AN F 14 10912/110.

169. Armengaud Aîné. Chemins de fer atmosphériques. Publication industrielle des machines, outils et appareils les plus perfectionnés... op. cit. (note 51), p. 77-84. À propos du système Arnollet, Armengaud reproduit le rapport fait par Gabriel Lamé à l'Académie des Sciences le 7 avril 1845, rapport qui connut une traduction en langue anglaise faite par un ingénieur de Dublin, Robert Mallet (membre de l'Institution of Civil Engineers), et publiée dans Quarterly Papers on Engineering, Edited and Published by John Weale. London : vol. V, 1846, p. 3-16. Ce Robert Mallet (à ne pas confondre avec le Jacques Mallet français) revendique l'antériorité de l'invention des réservoirs d'air brevetée par Arnollet et dit l'avoir présentée, en 1842, à James Pim et Jacob Samuda: MALLET, Robert. Three reports upon improved methods of constructing and working atmospheric railways. London : 10 juin 1845 [ICE].

170. Sur François Cavé (1794-1875), l'un des fournisseurs de locomotives pour la compagnie de Paris à Saint-Germain (locomotives baptisées Gauloise et Dorade) et, par ailleurs, constructeur de bateaux et de machines à vapeur pour la navigation, voir CALLA [François-Christophe]. Rapport fait par M. Calla au nom du comité des arts mécaniques sur plusieurs établissements affectés à la construction des grandes machines à vapeur et des machines locomotives. Bulletin de la Société d'Encouragement pour l'Industrie nationale. Décembre 1842, n CCCCLXII, p. 467-484 ; PAYEN, Jacques. La machine à vapeur fixe, op. cit. (note 49), p. 106-107, et BELHOSTE, Jean-François, LOHR, Évelyne, SMITH, Paul. Paris, ville d'usines au XIX siècle. BOWIE, Karen (dir.). La modernité avant Haussmann, formes de l'espace urbain à Paris, 1801-1853. Paris : Éditions Recherches, 2001, p. 338-352.

171. Arnollet, Pierre-Jean-Baptiste. Chemins de fer atmosphériques, Lettre à $M$. le Ministre des Travaux Publics par M. Arnollet, ingénieur en chef des Ponts-et-Chaussées, en retraite. Paris : Imprimerie Lange Lévy et Cie, 1844 (lettre datée du 7 avril 1844, p. 7). Né en 1776, PierreJean-Baptiste Arnollet avait participé, en tant qu'ingénieur, à l'expédition d'Égypte, passant ensuite l'essentiel de sa carrière comme ingénieur en chef des Ponts et Chaussées dans le département de la Côte d'Or. En 1844 et 1845, sa retraite lui laissait le loisir de rédiger de nombreuses brochures en défense de son système, la plupart se plaignant du peu d'intérêt manifesté à son égard par le ministre des Travaux publics. En janvier 1846, afin de faire la preuve de l'imperméabilité de ses réservoirs, il réclama un essai de son système sur un chemin atmosphérique de $1500 \mathrm{~m}$ de longueur entre la Villette et le plateau de Belleville: Arnollet, Pierre-Jean-Baptiste. Chemins de fer atmosphériques, Mémoire sur les perfectionnements d'après lesquels ces chemins peuvent être établis et exploités facilement, et à peu de frais, dans tous les lieux accessibles aux voitures ordinaires; et donner dans les plaines un produit net trois fois plus grand que celui qu'on obtiendrait du système à locomotives, adressé à $M$. le ministre des Travaux publics le 18 janvier [1846] par M. Arnollet, ingénieur en chef des Ponts et Chaussées, en retraite. Paris : Imprimerie Langé Lévy et Cie, 1846.

172. «Je propose d'effectuer la connexion entre le voyageur dynamique et le gouverneur au moyen de l'attraction magnétique. Des aimants en fer à cheval de grande taille et de grande puissance parcourent le tube près de la surface mais sans contact; sous le gouverneur, une combinaison similaire d'aimants passent par dessus le tube sans contact [...] la puissance électromagnétique peut également être employée... » Pinkus, Specifications, "An improved method of or apparatus for communicating and transmitting or extending motive power... », 30 août 1834 [ICE].

173. Chemin de fer électro-pneumatique proposé par M. Jobard, directeur du musée de l'industrie, imprimé (s.l., s.d.) de 7 pages avec une planche, tiré du Bulletin du musée de 
l'Industrie, 1846 [ICE], texte également reproduit dans Armengaud Aîné, Chemins de fer atmosphériques. Publication industrielle des machines, outils et appareils les plus perfectionnés..., op. cit. (note 51), p. 110-113.

174. Dans son discours à la Chambre du 2 juillet 1844, Arago évoque aussi cette idée : «On a imaginé des communications magnétiques, mais c'étaient des rêves. On pourrait produire ainsi des effets insignifiants à figurer dans les cours de physique, rien de plus. » (Moniteur Universel, 4 juillet 1844).

175. VERNE, Jules. Paris au $X X X^{\mathbf{e}}$ siècle. Paris : Hachette/Le Cherche Midi Éditeur (Livre de poche), 1994, p. 41.

176. «Quant à la soupape, tous les ingénieurs et mécaniciens tant français qu'étrangers, qui ont été à même de comparer celle de MM. Clegg et Samuda à celle à lames d'acier de M. Hédiard, avec les nouveaux perfectionnements qu'il vient d'y apporter, ne font plus aucun doute sur l'emploi tout à fait pratique de cette dernière. » DUBERN, H. A. De l'application de l'air atmosphérique aux chemins de fer ; résumé des opinions des ingénieurs français et anglais sur les chemins de fer atmosphériques. Paris : À la librairie industrielle-scientifique de L. Mathias, 1846, p. 80.

177. Sur la banque de Jacques Ardoin (1779-1854) et de son fils Jules (1822-1871), grand propriétaire immobilier à Saint-Ouen et l'un de ses conseillers municipaux, voir STOSKOPF, Nicolas. Les Patrons du Second Empire, Banquiers et financiers parisiens. Paris : Picard/ Éditions Cenomane, 2002, p. 71-73.

178. VUIGNER, Émile. Notice sur un chemin de fer d'essai établi à Saint-Ouen pour expérimenter la soupape longitudinale Hédiard dans le système de propulsion atmosphérique. Paris: Typographie Lacrampe et Cie, 1846 (texte daté du 15 avril 1846). Ce chemin de Saint-Ouen est également décrit dans un manuscrit de 19 folios, dû à Jacques-Eugène Armengaud (Armengaud aîné), conservé à Saint-Denis dans le portefeuille des machines du CNAM (Inv. 13571.693).

179. Sur l'entreprise de François-Étienne Calla (1760-1836) et de son fils François-Christophe (1802-1884), également réputée pour ses machines textiles et pour ses fontes d'art, voir PAYEN, Jacques. La machine à vapeur fixe, op. cit (note 49), p. 143 et DAUMAS, Maurice. Les mécaniciens autodidactes français et l'acquisition des techniques britanniques. L'acquisition des techniques par les pays non-initiateurs, Pont-à-Mousson, 28 juin-5 juillet 1970. Paris: Éditions du CNRS, 1973 (colloques internationaux du CNRS, n 538, organisé avec la collaboration du International Co-operation in History of Technology Committee ICOHTEC), p. 301-334.

180. L'Illustration en rend compte dans son numéro du 22 août 1846, trouvant le système Hédiard « bien supérieur au système Clegg et Samuda ».

181. BAUDE, Louis-Alphonse. Rapport sur un chemin de fer d'essai établi à Saint-Ouen pour expérimenter la soupape longitudinale de $\mathrm{M}$. Hédiard, dans le système de propulsion atmosphérique (rapport daté du 30 août 1848). Bulletin de la Société d'Encouragement pour l'Industrie nationale, octobre 1848, $\mathrm{n}^{\circ}$ DXXXII, p. 615-634.

182. CALLITE, Anne. Alexis Hallette, ingénieur et industriel en Artois, 1788-1846. s.l., s.d., [Éditions du Geai-Bleu, Roubaix, 2003], (ouvrage publié avec le concours du Conseil régional du Nord Pas-de-Calais), préface de François Caron, p. 2-3 ; voir aussi la thèse citée de CALLITE, Anne. La construction du matériel roulant ferroviaire en France, l'exemple nordiste (1828-1939), p. 106-111 et 151-192; sur Hallette, voir aussi PAYEN, Jacques. La machine à vapeur fixe, op. cit., p. 98-100.

183. CALLA [François-Christophe]. Rapport cité (note 170). Bulletin de la Société d'Encouragement pour l'Industrie nationale. Décembre 1842, n CCCCLXII, p. 467-484.

184. L'Illustration, 15 février 1847. Cet article, publié après le décès d'Alexis Hallette, est très admiratif de l'homme - « véritable soldat industriel » - et de son usine, « un modèle à citer »; il est illustré de deux gravures d'après Bonhommé. 
185. COTTE, Michel. Le choix de la révolution industrielle, op. cit. (note 1), p. 101, 322-323 et 436.

186. Cité par CALLITE, Anne, op. cit. (note 182), p. 59.

187. HALLETTE, Alexis. Tube-propulseur Hallette, système d'exécution et d'exploitation des chemins de fer par la pression atmosphérique. Paris-Batignolles : Imprimerie Hennuyer et Turpin, 1844. Cette publication comporte des renseignements préliminaires, remontant à Papin puis à l'invention de l'ingénieur danois Medhurst (sic) et citant le rapport de Teisserenc de mars 1843. La première note est datée du 10 février 1844. La seconde est sans date, mais rédigée peu après la parution du rapport Mallet. Des exemplaires de cette brochure se trouvent dans les archives de l'Institution of Civil Engineers, ainsi que dans AN F 1411171.

188. CALLITE, Anne, op. cit. (note 182), p. 60.

189. OLIVIER, Théodore. Rapport fait au nom du comité des arts mécaniques sur le système de chemin de fer atmosphérique de M. Hallette, à Arras. Bulletin de la Société d'Encouragement pour l'Industrie nationale, mai 1846, $\mathrm{n}^{\circ} \mathrm{DIII}, \mathrm{p} .219-224$.

190. MALLET, « Rapport sur les expériences faites à Arras pour juger les procédés appliqués par Mr Hallette à la propulsion atmosphérique... », AN F 14 11171. Ce rapport multigraphié de 32 pages est partiellement reproduit dans Armengaud aîné, complété de quelques annotations techniques de Hallette lui-même ; Armengaud Aîné, Chemins de fer atmosphériques. Publication industrielle des machines, outils et appareils les plus perfectionnés..., op. cit. (note 51), p. 129-137.

191. Lettre de Hallette à Mallet, Arras, 5 avril 1844, AN F 14 11171. Le colonel Répécaud, de l'Académie d'Arras, notait également l'incertitude qui demeurait au sujet du tissu formant l'enveloppe des lèvres: REPECAUD. Mémoire sur l'emploi de la pression atmosphérique, op. cit. (note 143), p. 8.

192. Découverte aux États-Unis par Charles Goodyear, la «vulcanisation » du caoutchouc aurait été baptisée et brevetée à Londres, par Thomas Hancock, en 1843.

193. Un peu à l'instar de l'homme qui inventa l'essieu, sans savoir ce qu'il pourrait bien mettre à chaque extrémité, Hallette donne l'impression ici d'avoir inventé le pneu, sans penser l'adapter à la jante d'une roue.

194. Journal des Chemins de Fer, 8 mars 1845.

195. Remarque faite par un membre du Conseil des Ponts et Chaussées dans la séance du 7 juillet 1845, AN F 14 10912/109.

196. «MM. Harlé, Esnault, Delessert, Delebecque, députés du Pas-de-Calais, recommandent l'emploi du système Hallette pour le chemin de fer ", courrier en date du 16 mai 1845 enregistré à l'administration des Ponts et Chaussées, "Sommier des chemins de fer », cité par VILLEDEUIL, Pierre-Charles-Laurent de. Euvres de Émile et Isaac Pereire, op. cit. (note 130), tome III, p. 1985.

197. L'Illustration, 12 octobre 1844 : «M. Hallette, d'Amiens (sic) a répondu de la façon la plus victorieuse aux objections adressées à la soupape irlandaise, en lui substituant un système tout à fait nouveau et fort ingénieux [...] À ce perfectionnement principal [les lèvres pneumatiques], $M$. Hallette a joint une foule d'autres. Son système est assez neuf pour qu'il lui soit donné à juste titre le nom de système français. »

198. HADFIELD, Charles. Atmospheric Railways, op. cit. (note 24), p. 84. Une note parue dans le Journal des Chemins de Fer du 11 juillet 1846, parle de cette compagnie et de son spécimen construit à Peckham. « La compagnie anglaise a bien compris la valeur de ce système [...] elle est en mesure de traiter pour son application en France, Autriche, Belgique et Hollande. " Une circulaire signée du secrétaire de la compagnie, Edwin J. Coley, et datée du 28 février 1846, est conservée dans les collections du National Railway Museum à York (1998-9818). Elle annonce l'acquisition d'un terrain au Rosemary Branch Peckham et l'installation des rails et du tube de propulsion; des essais du système sont annoncés pour la semaine suivante. Ses avantages : « une 
grande économie comparée aux autres systèmes atmosphériques; une diminution des frais dans l'acquisition des terrains, des travaux de déblaiement et de tunnel n'étant que rarement nécessaires; considérable réduction dans le poids des rails qui doivent être fabriqués à l'heure actuelle pour résister au poids et aux tremblements (quivering) des locomotives; départs fréquents ; augmentation de vitesse ; moins d'oscillations ; absence de bruit, chaleur, cendres et fumée ; aucune possibilité de collision ; sécurité complète pour les voyageurs ; aucun accident ni aux personnes ni à la propriété par feu ou par l'éclatement des chaudières ou par la rupture d'autres éléments de la locomotive. »

199. CALLITE, Anne. op. cit. (note 182), p 77.

\section{RÉSUMÉS}

Au cours des années 1840, au moment où se met en place le réseau ferré de France, rayonnant de la capitale, la traction par des locomotives à vapeur, pour différentes raisons d'économie et de sécurité, suscite encore réticences et critiques. Cet article veut remettre en lumière un système alternatif de propulsion par la pression de l'air, système qui fait naître en France, en Angleterre et ailleurs de grands espoirs, partagés non seulement par d'éminents ingénieurs mais aussi par des autorités scientifiques et administratives, la presse et le public. Il propose un récit de son application sur quatre lignes exploitées commercialement, en se focalisant, dans la deuxième partie du texte, sur la version française, l'extension de la ligne de Paris à Saint-Germain, où le système atmosphérique fonctionna de 1847 à 1860. Il cherche à restituer l'enthousiasme et les débats autour de ces chemins de fer atmosphériques à une époque où l'on ne savait pas encore qu'il s'agissait d'une impasse technologique. Chemin faisant, et comme cas d'étude, il essaie de suivre la circulation, essentiellement à travers la Manche, des idées techniques qui portaient les essais entrepris, puis leur abandon.

During the 1840s, as the French national railway network, centred on Paris, gradually took form, steam locomotion, for reasons of economy and security, still inspired much hesitation and criticism. This article gives an account of an alternative form of propulsion by air pressure which, in France, in England and elsewhere, provoked considerable enthusiasm, not only amongst eminent engineers, but also in scientific and administrative circles, the press and the public. It gives a brief account of the four lines exploited commercially, concentrating, in the second part of the text, on the French one, the extension of the Paris - Saint-Germain railway, which was operated atmospherically from 1847 to 1860 . It tries to suggest something of the high hopes and the debates which accompanied the new system before it turned out to be a technological dead-end. Along the way, it also looks at how technical information circulated at the time, essentially back and forth across the Channel, making the experimental lines possible, and then putting an end to them. 


\section{INDEX}

Keywords : Eugène Flachat (1802-1873), Alexis Hallette (1788-1846), atmospheric railway, Charles Vignoles (1793-1875), compressed air, Ireland, Isambard Kingdom Brunel (1806-1859), Jacob Samuda (1811-1844), Joseph d'Aguilar Samuda (1813-1885), Kingstown-Dalkey railway, London , Paris - Saint-Germain-en-Laye railway, Pereire brothers (Émile 1800-1875 et Isaac 1806-1880), railway heritage, Samuel Clegg (1781-1861), South Devon railway, William Cubitt (1785-1861) Mots-clés : air comprimé, Alexis Hallette (1788-1846), Charles Vignoles (1793-1875), chemin de fer atmosphérique, chemin de fer de Kingstown à Dalkey (Irlande), chemin de fer de London , chemin de fer de Paris à Saint-Germain-en-Laye, chemin de fer de South Devon, Eugène Flachat (1802-1873), Frères Pereire (Émile 1800-1875 et Isaac 1806-1880), Isambard Kingdom Brunel (1806-1859), Jacob Samuda (1811-1844), Joseph d'Aguilar Samuda (1813-1885), patrimoine ferroviaire, Samuel Clegg (1781-1861), William Cubitt (1785-1861)

\section{AUTEUR}

\section{PAUL SMITH}

Ingénieur d'études, sous-direction de l'archéologie, de l'ethnologie, de l'inventaire et du système d'information, direction de l'architecture et du patrimoine, ministère de la culture et de la communication.paul.smith@culture.gouv.fr 